\title{
Pre-flight Calibration and Near-Earth Commissioning Results of the Mercury Plasma Particle Experiment (MPPE) Onboard MMO (Mio)
}

\author{
Yoshifumi Saito - Dominique Delcourt - Masafumi Hirahara. Stas Barabash • \\ Nicolas André - Takeshi Takashima et al. [full author details at the end of the article]
}

Received: 17 June 2020 / Accepted: 19 June 2021

(c) The Author(s), under exclusive licence to Springer Nature B.V. 2021

\begin{abstract}
BepiColombo Mio (previously called MMO: Mercury Magnetospheric Orbiter) was successfully launched by Ariane 5 from Kourou, French Guiana on October 20, 2018. The Mercury Plasma/Particle Experiment (MPPE) is a comprehensive instrument package onboard Mio spacecraft used for plasma, high-energy particle and energetic neutral atom measurements. It consists of seven sensors including two Mercury Electron Analyzers (MEA1 and MEA2), Mercury Ion Analyzer (MIA), Mass Spectrum Analyzer (MSA), High Energy Particle instrument for electron (HEP-ele), High Energy Particle instrument for ion (HEPion), and Energetic Neutrals Analyzer (ENA). Significant efforts were made pre-flight to calibrate all of the MPPE sensors at the appropriate facilities on the ground. High voltage commissioning of MPPE analyzers was successfully performed between June and August 2019 and in February 2020 following the completion of the low voltage commissioning in November 2018. Although all of the MPPE analyzers are now ready to begin observation, the full service performance has been delayed until Mio's arrival at Mercury. Most of the fields of view (FOVs) of the MPPE analyzers are blocked by the thermal shield surrounding the Mio spacecraft during the cruising phase. Together with other instruments on Mio including Magnetic Field Investigation (MGF) and Plasma Wave Investigation (PWI) that measure plasma field parameters, MPPE will contribute to the comprehensive understand-
\end{abstract}

The BepiColombo mission to Mercury

Edited by Johannes Benkhoff, Go Murakami and Ayako Matsuoka

BepiColombo Mio/MPPE Team: Y. Saito, M. Hirahara, S. Barabash, D. Delcourt, A. Coates, N. André, T. Takashima, K. Asamura, C. Aoustin, J.-A. Sauvaud, P. Louarn, M. Blanc, C. Jacquey, C. Mazelle, I. Dandouras, V. Genot, D. Toublanc, C. Peymirat, A. Fedorov, E. Amata, R. Bruno, M.B. Cattaneo, G. Consolini, M.F. Marcucci, Z. Nĕmeček, B. Lavraud, L. Griton, S. Aizawa, H.-C. Seran, J. Rouzaud, Q.M. Lee, E. Le Comte, E. Penou, M. Petiot, D. Moirin, S. Machida, I. Shinohara, W. Miyake, T. Terasawa, C. Owen, A. Fazakerley, T. Nagatsuma, K. Seki, T. Nagai, A. Ieda, H. Hasegawa, J.-M. Illiano, J.-J. Berthelier, D. Fontaine, N. Krupp, J. Woch, S. Yokota, M. Fraenz, H. Krueger, H. Michalik, L. Hadid, R. Modolo, B. Fiethe, B. Katra, F. Leblanc, C. Verdeil, H. Fischer, J.-D. Techer, D. Reisenfeld, R. Elphic, H. Funsten, D. McComas, M. Grande, H. Matsumoto, T. Yanagimachi, T. Obara, Y. Miyoshi, Y. Ebihara, M. Nose, F. Tsuchiya, T.A. Fritz, Q. Zong, T. Mitani, S. Kasahara, M. Shimoyama, Y. Kazama, M. Yamauchi, M. Holmström, Y. Futaana, R. Lundin, P. Wurz, M. Wieser, H. Andersson, S. Karlsson, W. Benz, W.-H. Ip, L.-N. Hau, M. Hoshino, M. Fujimoto, K. Maezawa, N. Terada, P. Trávniček, R. Smets, R. Modolo, F. Leblanc, R. Lallement, L. Zelenyi, H. Malova, M.N. Nishino, Y.-C. Wang, M. Oka, M. Yagi, Y. Harada, L. Xie, J. Zhong, J. Vaverka, K. Keika, W. Sun, L. Wang 
ing of the plasma environment around Mercury when BepiColombo/Mio begins observation after arriving at the planet Mercury in December 2025.

Keywords Mercury · Magnetosphere · Solar wind · Exosphere · Ion · Electron · Energetic neutral atom

\section{Introduction}

Our knowledge of Mercury's plasma environment has significantly increased during the past decade owing to new observations made by the Mercury orbiter MESSENGER. However, many questions remain. To provide greater detail on this plasma environment, BepiColombo Mio was successfully launched by Ariane 5 from Kourou, French Guiana on October 20, 2018 as part of a joint mission between European Space Agency (ESA) and Institute of Space and Astronautical Science/Japan Aerospace Exploration Agency (ISAS/JAXA).

When BepiColombo Mission began about 15 years ago, Mercury was one of the least explored planets of our solar system. No spacecraft had visited Mercury since Mariner 10 made three fly-bys past the planet in 1974 and 1975. Mariner 10 discovered that Mercury possesses an intrinsic magnetic field with very weak intensity compared with that of other magnetized planets in our solar system (Ness et al. 1974; Ogilvie et al. 1974). About 30 years after Mariner 10 visited Mercury, MESSENGER made its first fly-by observation in 2008. In 2011, MESSENGER was inserted into Mercury's orbit to become the world's first Mercury orbiter, which continued observation for more than four years.

Mariner 10 discovered the dominance of the dipole term in the spherical harmonic expansion of Mercury's magnetic field. This suggests that the interaction between the solar wind and Mercury's magnetosphere should be "Earth-like", in contrast to the cases of Mars and Venus in which the planetary magnetic fields have negligible intensity or have only local effects on the interaction. The MESSENGER observation revealed that the dipole moment of Mercury is deviated from its center northward by about 20\% of the planet's radius (Anderson et al. 2011). Because the magnetic field reflects the internal structure and its dynamics, detailed observation of Mercury's magnetic field is one of the most important targets of BepiColombo.

Mercury's small size and low gravity result in environmental characteristics that differ significantly from those of Earth. MESSENGER clearly showed the extremely dynamic behavior of Mercury's magnetosphere where substorm-like phenomena repeat with very short time scales (Imber and Slavin 2017). MESSENGER also proved the existence of large amounts of heavy elements in the magnetosphere (Zurbuchen et al. 2011). BepiColombo/Mio will make exhaustive measurements of Mercury's magnetosphere including comprehensive measurements of plasma and particles. Such observation of plasma and particles from spinning spacecraft covering a $4 \pi$ FOV with a time resolution as high as a few seconds will reveal the mechanism of the substorm-like phenomena occurring in Mercury's magnetosphere to clarify similarity and difference between Earth and Mercury. In addition, the ion energy mass spectrometer on Mio has high mass resolution that can distinguish between the species of planetary heavy ions. This will help to explain the contribution of heavy ions on the magnetospheric processes in Mercury's magnetosphere.

The Mercury Plasma/Particle Experiment (MPPE) is a comprehensive instrument package used for plasma, high-energy particle and energetic neutral atom measurements. It consists of seven sensors including two Mercury Electron Analyzers (MEA1 and MEA2), Mercury Ion Analyzer (MIA), Mass Spectrum Analyzer (MSA), High Energy Particle instrument for electron (HEP-ele), High Energy Particle instrument for ion (HEP-ion), and Energetic Neutrals Analyzer (ENA). 
Together with other instruments onboard Mio including Magnetic Field Investigation (MGF) and Plasma Wave Investigation (PWI) that measure plasma field parameters, MPPE will contribute to the comprehensive understanding of the plasma environment around Mercury when BepiColombo/Mio begins observation after arriving at the planet Mercury in December 2025.

\section{Science Objectives of MPPE}

\subsection{Structure, Dynamics, and Physical Processes Occurring in Mercury's Magnetosphere}

Because the intrinsic magnetic field is weaker and the dynamic pressure of the solar wind is stronger at Mercury than at Earth, solar wind can sometimes directly interact with the dayside planetary surface in the low-latitude region. Recent MESSENGER observations indicate that the high dynamic pressure of the solar wind causes the compression and magnetic flux transfer by reconnection, which can completely erode the dayside magnetosphere (Slavin et al. 2019). Even when the solar wind dynamic pressure is not so strong, the solar wind plasma can directly penetrate until reaching the planetary surface through the cusp regions. Another important characteristic of Mercury's magnetic field is the offset of the dipole. The fly-bys by Mariner 10 suggested the possibility of northward offset of the magnetic dipole by $0.2 R_{M}$ (Whang 1977), which was confirmed by MESSENGER observations (Anderson et al. 2011). This means that the planetary magnetic field at the surface is stronger at the northern pole than at the southern pole, and that the solar wind plasma can more easily access the planetary surface in the southern polar region. In addition, heavy ions and neutrals sputtered from the planetary surface are major observation targets of MPPE. The effects of the direct interaction of the space plasma and the planetary surface on the remaining planetary processes can be investigated only in Mercury's environment.

Despite the qualitative similarities of the global structures of the Mercury and Earth magnetospheres, many differences remain between them. The small dimensions of Mercury's magnetosphere imply short time scales of the dynamic phenomena occurring therein. The magnetospheric convection, potentially driven by dayside reconnection, is expected to complete its circulation within just a few minutes, which is only $1 / 30$ of the corresponding time scale at Earth (e.g., Slavin 2004). Flux transfer events (FTEs) are frequently detected in rapid succession at Mercury's magnetopause with much shorter time scales than those at Earth (Slavin et al. 2012; Imber et al. 2014). The small magnetosphere also implies that kinetic behavior of plasma is particularly important. Because the typical scale of Mercury's magnetospheric structures are on the order of the proton Larmor radius, the ideal magnetohydrodynamics (MHD) approximation could be inadequate for describing the global dynamics of the magnetosphere. This is exemplified by MESSENGER observations, which showed that a typical proton gyroradius in Mercury's plasma sheet is $\sim 380 \mathrm{~km}$ (DiBraccio et al. 2015) and that the thickness of the plasma sheet is comparable to the proton gyroradius (Sun et al. 2017). For zero or weak guide magnetic fields, ion scale current sheets with a thickness comparable to the ion inertia length or the ion Larmor radius are predicted to become highly unstable for the current driven instabilities, which lead to quick triggering of magnetic reconnection (Shinohara and Fujimoto 2005).

Many questions remain about the substorms in Mercury's magnetosphere. Because the concept of storage and sudden release of energy is likely universal, efforts to answer these questions enable us to examine the ubiquitous problems of magnetized plasmas. One of these questions is related to the dawn-dusk asymmetry in the plasma sheet associated with 
substorm activities (Sun et al. 2017). In particular, proton energization and heating through substorm activities occur more often on the dawnside than on the duskside, which is opposite that occurring in Earth's magnetosphere. The cause of the dawn-dusk asymmetry remains to be studied using BepiColombo data. The questions related to the Mariner 10 observation events such as drift echoes have been considered in the context of substorms. These particle phenomena need to be studied with an unbiased attitude and a complete field of view. Specifically, all substorm-like events at Mercury should be studied in the context of solar wind-magnetosphere interaction and particle acceleration processes. In this context, measurements such as those by MPPE will be of paramount importance.

Recent theoretical studies suggest that efficient plasma transport can be achieved within highly rolled-up vortices that form owing to the velocity shear at the tail-flank boundary (Hasegawa et al. 2004; Nakamura et al. 2017). It is widely accepted that Kelvin-Helmholtz $(\mathrm{K}-\mathrm{H})$ instability operates at Earth's magnetopause and plays a significant role in transporting mass and energy from the solar wind to the magnetosphere. MESSENGER observations showed that K-H vortices develop predominantly on the duskside (Gershman et al. 2015). $\mathrm{K}-\mathrm{H}$ waves as sources of these vortices are also detected mainly at dusk (Liljeblad et al. 2014). A possible contribution of heavy ions to K-H instability will be explored by analyzing the BepiColombo data.

The study of large amplitude electromagnetic waves around the magnetopause is also important in terms of particle transport, diffusion and acceleration. Anisotropic particle distribution and accelerated particle beams can excite various electromagnetic waves. The mapping of characteristic waves and particle velocity distribution functions, and their comparison with those found on other planets are also important.

The existence of Na ions in Mercury's magnetosphere is another interesting factor. The heavy mass of these ions combined with the weak magnetic field result in large Larmor radii in the Mercury magnetosphere. The turbulence discussed here is basically of MHD nature but the large Larmor radius is comparable to the scale of the vortices and efficient heating of $\mathrm{Na}$ ions by turbulence is expected. Heated $\mathrm{Na}$ ions carry non-negligible pressure and thus play a role in determining the shape and dynamics of the magnetosphere (Gershman et al. 2014). Such a significant contribution of heavy ions would be an analog to large storms in Earth's magnetosphere although this could be the average state of the Mercury magnetosphere. Comprehensive observations of both the tail-flank turbulence and the large-scale convection powered by the reconnection in the small-scale magnetosphere are thus quite interesting from the perspectives of basic magnetohydrodynamics and magnetospheric physics. MPPE includes required plasma detectors with good time resolution and a mass spectrometer with sufficiently wide energy coverage.

The energization mechanism of magnetospheric plasma at Mercury has been unsolved since the Marinar 10 era. Although intense bursts of energetic charged particles $>35 \mathrm{keV}$ likely associated with substorm activities have been detected by Mariner 10 (Simpson et al. 1974), their species, flux, and energy spectrum for the events were not precisely determined owing to instrument limitations. Recent observations by EPS onboard MESSENGER revealed that the bursts of energetic charged particles are composed of high-energy electrons (Ho et al. 2011). This finding combined with the indications of MESSENGER/GRNS data suggest that the major components of the energetic bursts are electrons of several tens to $\sim 100 \mathrm{keV}$ (Slavin et al. 2018). Although the most plausible mechanism of the electron energization is magnetic reconnection in Mercury's magnetotail associated with substorm activities, the lack of low-energy electron observations by MESSENGER prevents us from making a conclusion on this topic. However, observations of electrons in a wide energy range by MPPE and other instruments will enable us to identify the generation mechanisms of the high-energy electrons around Mercury. 


\subsection{Interaction Among Magnetosphere, Exosphere, Surface, and Interior of Mercury}

The Mercury environment can be characterized by complicated interaction among the surface, exosphere, and magnetosphere (Milillo et al. 2005). The lack of a thick atmosphere allows the space plasma to directly reach the surface. The surface materials are then ejected into space to form the exosphere, part of which is ionized and governed by the electromagnetic environment of Mercury or magnetosphere. This complex system occurs in all objects in the Solar System with no atmosphere. In particular, the Moon provides an appropriate environment for understanding this coupling (e.g., Futaana et al. 2018). Although it does not have a global magnetic field, the Moon's lacks of atmosphere and strong ionosphere affects the plasma-surface interaction, exosphere formation, and interaction with the upstream plasma. However, part of the Moon is magnetized where the dynamic plasma physics is in operation. Recent measurements of the Moon by several orbiters and fly-bys (e.g., Nozomi, Lunar Prospector, Kaguya, Chang'E, Chandrayaan-1, ARTEMIS, and LADEE) have significantly increased our knowledge of the lunar environment, part of which can be applied to understand these interactions at Mercury.

Mercury's surface releases $\mathrm{Na}, \mathrm{O}, \mathrm{H}, \mathrm{He}, \mathrm{K}, \mathrm{Ca}$, and possibly other compositions by photon-stimulated desorption, thermal desorption, micrometeoric impacts, chemical sputtering, and ion sputtering, to form a highly extended tenuous atmosphere or exosphere. The exosphere of Mercury will be investigated by instruments on MPO with a relatively lower orbiting altitude. However, the exospheric ions circulate along the convection of the magnetosphere and then partially re-enter Mercury's surface-exosphere system to further sputter the surface material. Conversely, the exospheric ions affect the magnetospheric convection. These facts indicate that Mercury's exosphere is not a single system, rather it strongly interacts with other regions, constituting a surface-exosphere-magnetosphere system. Among the release processes, ion sputtering is mostly related to magnetospheric processes. As a result of magnetospheric dynamics, the magnetospheric and solar wind ions precipitate on Mercury's surface resulting in atom and ion sputtering (Killen et al. 2001). The sputtered ions and exospheric photoions feed the magnetosphere, which affects its dynamics.

Ions originating directly from solar wind, those accelerated in the tail, and energized planetary ions, all precipitate onto Mercury's surface, which results in extensive sputtering (Grande 1997; Wurz and Lammer 2003; Mura et al. 2009). No reservoir of trapped particles exists near Mercury because the planet occupies a large portion of its inner magnetosphere; in this case, accelerated energetic particles hit the surface easily and become lost quickly (Delcourt et al. 2003; Yagi et al. 2017). The Mercury magnetosphere does not include a ring current region such as that present in Earth's inner magnetosphere which enables quasi-trapped $\mathrm{Na}$ ions to exist in the low-latitude region near the planet as indicated by simulation (Yagi et al. 2010) and MESSENGER observation (Schriver et al. 2011). The energetic particles in the magnetosphere should precipitate to the planetary surface/exosphere directly through pitch angle scattering by wave-particle interactions and field line curvature. The sputtering by particle precipitation is an escape process of heavy ions from the planetary surface: the direct interaction of precipitating particles with the planetary surface is important for the evolution of particle circulation in the Mercury magnetosphere (Ip 1986). Therefore, investigation of the loss processes of high-energy particles and the relationship between the energetic particle and the planetary surface is also an important objective of the MPPE observations. The integrated energy spectrum of the sputtered products falls off as $\mathrm{E}^{-2}$, reflecting the Thompson-Sigmund formula (e.g., Sigmund et al. 1982) and results in relatively high fluxes at energies greater than $10-100 \mathrm{eV}$ (Massetti et al. 2003). Measuring these low-energy neutral atoms (LENA) by MPPE-ENA while monitoring precipitating ions 
by MPPE-MSA and MPPE-MIA are crucial for understanding the contribution of sputtering to the formation of Mercury's exosphere, which reveals dynamical and spatial variations of the sputtering source (Fatemi et al. 2020).

In addition to magnetosphere-exosphere coupling, MESSENGER observation revealed a possible magnetosphere-exosphere-interior system. The MESSENGER/MAG observation provided evidence of field aligned currents (FACs) at Mercury, where a weak Region 1 current system exists but no Region 2 current does (Anderson et al. 2014). The existence of a Region 1 current system suggests the possibility of electric current closure through conductive material at the depth of the outer core, which strongly depends on the electric conductivity at the planet's surface and interior region. Direct measurement of FAC carriers by MPPE is highly anticipated. In addition, the balance between the magnetic reconnection and induction at the dayside magnetopause could provide clues for understanding the planetary interior (Heyner et al. 2016).

The dynamic response of the Mercury magnetosphere to solar wind variation is sometimes regarded as a possible explanation for the variability in the Na exosphere both spatially and temporally on timescales less than one day as observed by ground-based remote sensing measurement. Model calculations show that solar wind ions and the exospheric ions energized in the magnetosphere very non-uniformly affect Mercury's surface, which includes various impact regions such as auroral impact, cusp impact, and nose impact regions (Kallio and Janhunen 2003; Delcourt et al. 2003). However, such calculations also indicate that the impact regions and the effects of ion flux are rather sensitive to the magnetospheric dynamics and particle environment. MESSENGER discovered an X-ray aurora accompanied by electron precipitation (Lindsay et al. 2016; Dewey et al. 2017). Observation of precipitating electrons by MPPE-MEA and MPPE-HEP-ele will also contribute to understanding the magnetosphere-exosphere coupling.

The highly eccentric orbit of Mercury generates significant variation in the planetary environment between the perihelion and aphelion. Recent MESSENGER observations have shown that the $\mathrm{Na}$ exosphere is surprisingly constant in terms of annual variation, with no strong episodic variation or surface dependence noted (Cassidy et al. 2015). However, the Na intensity showed a strong seasonal variation that contradicts previous ground-based observation reported by Leblanc and Johnson (2010). The ionization frequencies of the exospheric neutrals by photoionization, electron impact ionization, and charge exchange interaction, which depend on the solar flux and the solar wind density and velocity, vary by a factor of two. These differences can cause a significant alteration of the dynamics of Mercury's magnetosphere-exosphere-surface-interior system. To understand this system, it is necessary to observe the planet's particle environment by MPPE observation for at least a few Mercury years.

\subsection{Shocks and the Inner Heliosphere}

A new era of inner heliosphere exploration began with the launch of the Parker Solar Probe in 2018 (Fox et al. 2016), followed by that of the Solar Orbiter in 2020 (Müller et al. 2013). Mio will play an important role in this heliosphere-wide, multi-mission exploration by its placement in Mercury's orbit. Following the successes of other missions such as Helios 1 and 2 in the 1980s, MESSENGER in 2008-2015, and the recent Parker Solar Probe, Mio is expected to achieve comprehensive in-situ measurement of plasmas in the innerheliosphere by taking advantage of the spin-stabilized configuration of the spacecraft and a suite of modern instruments. Mio is expected to make a wide range of discoveries regarding collisionless shocks, solar wind, pickup ions of interstellar-origin, solar energetic particles (SEPs), the modulation of galactic cosmic rays and other phenomena. 
Particles are accelerated to very high, non-thermal energies at astrophysical shocks, as evidenced by emission from astrophysical sources such as supernova remnants, extragalactic jets, and galaxy clusters. Particles are also accelerated at shocks in space such as planetary bow shocks and interplanetary shocks. In-situ measurement of shocks in various plasma environments is thus crucial for understanding of the generality and scaling-law of particle acceleration at shocks. Mercury is unique in that it is closer than any other planet to the Sun. Thus its orbit offers the greatest chance of detecting very fast interplanetary shocks particularly in extreme cases of solar eruptive events such as coronal mass ejection (CME) and solar flares. The shock speed can reach up to $4000 \mathrm{~km} / \mathrm{s}$ and the Alfvén Mach number can exceed several tens in value, as demonstrated combined observations and modeling (Smart and Shea 1985; Cliver et al. 1990). In fact, statistical analysis of MESSENGER data has confirmed that the shock transit speed is substantially higher in Mercury's orbit (Winslow et al. 2015) than that of Earth, which is consistent with earlier reports (Wang et al. 2005). Therefore, MPPE data combined with information obtained from other instruments on Mio will likely provide opportunities to study high-speed and/or high-Mach-number shocks well before they are substantially decelerated.

A key point in this research is that previously observed features of particle acceleration at shocks are often inconsistent with the standard diffusive shock acceleration (DSA) scenario. In DSA, the particle flux increases exponentially prior to the arrival of the shock, reaches its maximum at the shock front, and exhibits a power law with the power-law index as a function of the compression ratio only. In reality, however, shocks often do not exhibit a significant flux increase and, even if they are present, the power-law index does not match that predicted from the observed compression ratio. Such discrepancies have been reported for both ion acceleration (e.g., van Nes et al. 1984; Lario et al. 2003; Desai et al. 2004; Lario 2005; Fisk and Gloeckler 2012) and electron acceleration (Shimada et al. 1998; Ho et al. 2008). Moreover, for electrons, pre-energizing to non-thermal energies is required for the DSA process to begin, although the precise mechanism of such a process remains unclear (e.g., Tsurutani and Lin 1985; Oka et al. 2019; Amano et al. 2020). To address these problems, MPPE will provide comprehensive analyses of ion/electron velocity and pitchangle distributions, associated waves and turbulence, ion composition, and ion charge states before, during and after shock/CME passages.

The bow shock and magnetosheath signatures in Mercury's orbit are important targets of BepiColombo. The solar wind flow in Earth's orbit is usually super-Alfvénic with typical Alfvén Mach numbers of $5<M_{A}<10$. According to the standard solar wind model by Parker (1958), the Alfvén Mach number in Mercury's orbit is statistically lower than that in Earth's orbit (e.g., Slavin et al. 2018). We expect to detect super-Alfvénic solar wind with very low $M_{A}\left(1<M_{A}<2\right)$ and sub-Alfvénic solar wind $\left(M_{A}<1\right)$ in Mercury's orbit because BepiColombo will arrive at Mercury in 2025 when the solar activity is likely to be at its peak in Solar Cycle 25. For the bow shock under the lower but still super-Alfvénic solar wind $\left(M_{A}>1\right)$, the MESSENGER mission has already revealed significantly smaller magnetic "overshoots" (i.e., intensifications of the magnetic field magnitude within the shock transition layer) at Mercury's bow shock compared with that at Saturn (Masters et al. 2014). The differences in overshoot structure of the bow shocks is consistent with the expectations, which demonstrates the applicability of the scaling law based on the solar wind model. In addition, the lower $M_{A}$ solar wind yields unusual interaction between the magnetosheath and the magnetosphere, that depends strongly on the direction of the interplanetary magnetic field (IMF) (Lavraud and Borovsky 2008; Nishino et al. 2008). If the solar wind becomes sub-Alfvénic, the bow shock will alter to a slow-mode shock, and its shape and structure could differ significantly from that normally expected for a fast-mode bow shock (Hundhausen et al. 1987). An irregular bow shock can be detected by comparing the data with 


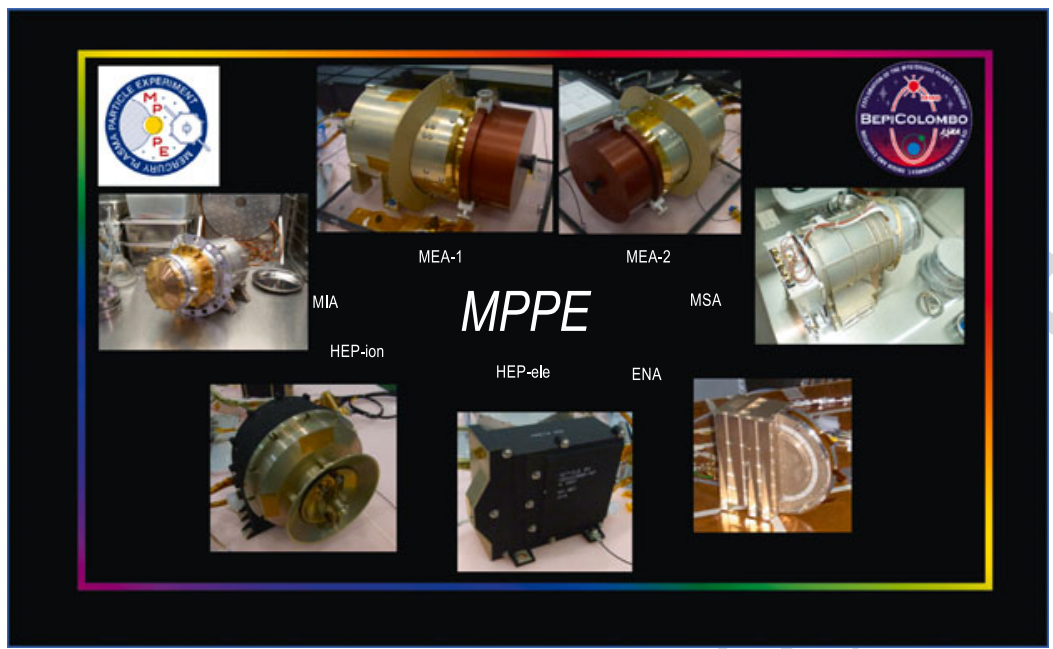

Fig. 1 Photo of the seven MPPE sensors

the typical shape/location of the bow shock established by MESSENGER (Winslow et al. 2013). Another candidate for sub-Alfvénic interaction of Mercury's magnetosphere with the extreme solar wind is the formation of Alfvén wings (Sarantos and Slavin 2009), which can be compared with sub-Alfvénic interaction of the Galilean moons with Jupiter's magnetosphere.

\section{Instrument Description and Pre-flight Calibration}

\subsection{Overview of MPPE Instrument Suite}

\subsubsection{MPPE Instrument Suite for Plasma/Particle Measurements}

As illustrated in Fig. 1, the MPPE suite is a comprehensive instrument package developed to achieve the scientific objectives described in Sect. 2. As previously discussed, it consists of seven sensors including MEA1, MEA2, MIA, MSA, HEP-ele, HEP-ion, and ENA (Saito et al. 2010a). These sensors measure plasma, high-energy particles and energetic neutral atoms with sufficiently high time resolution, wide energy and dynamic ranges, wide angle coverage, and high mass resolution.

Specifically, MEA1 and MEA2 measure the 3D phase space density of low energy electrons between $3 \mathrm{eV}$ and $26 \mathrm{keV}$ and were developed by the Research Institute in Astrophysics and Planetology (IRAP) in France. MIA measures 3D phase space density of low energy ions between $15 \mathrm{eV} / \mathrm{q}$ and $29 \mathrm{keV} / \mathrm{q}$ and was developed by ISAS/JAXA in Japan. MSA measures the mass identified 3D phase space density of low energy ions between $1 \mathrm{eV} / \mathrm{q}$ and $38 \mathrm{keV} / \mathrm{q}$ and was developed by the Laboratory of Plasma Physics (LPP) in France, the Max Planck Institute for Solar System Research (MPS) and Institute of Computer and Network Engineering (IDA)/Technical University of Braunschweig in Germany and ISAS/JAXA in Japan. HEP-ele and HEP-ion measure the energy spectra of high energy electrons between $30 \mathrm{keV}$ and $700 \mathrm{keV}$ and the mass identified ion energy spectra of high energy ions between $30 \mathrm{keV}$ and $1.5 \mathrm{MeV}$, respectively, and were developed by Institute for 


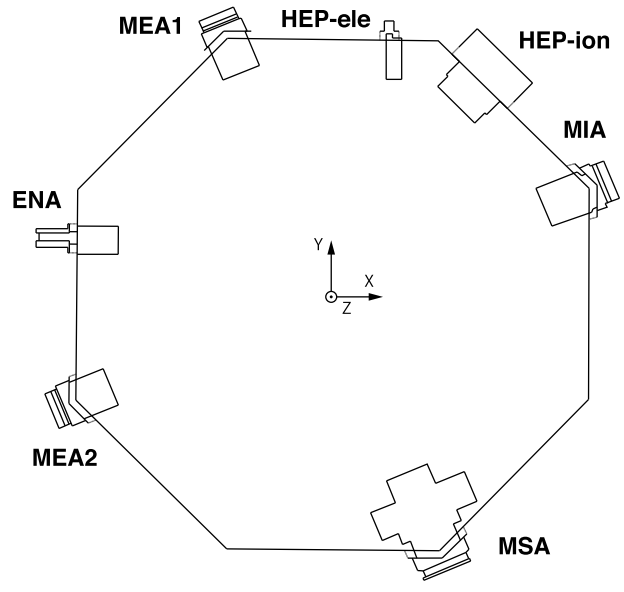

Fig. 2 Locations of the seven MPPE sensors
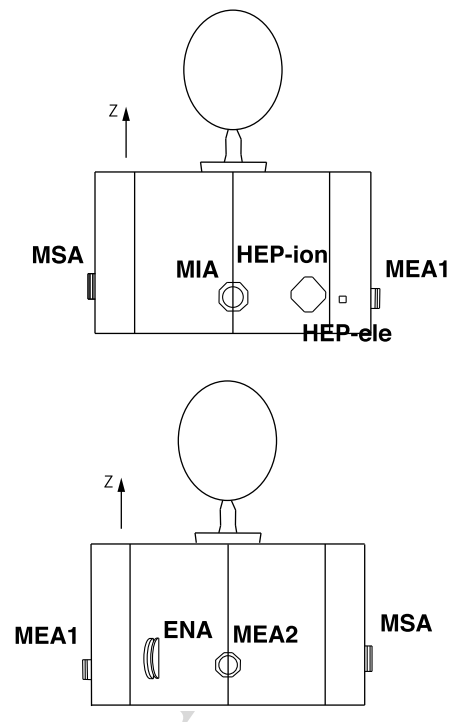

Space-Earth Environmental Research (ISEE)/Nagoya University and ISAS/JAXA in Japan. ENA measures the mass identified energetic neutral atoms between $10 \mathrm{eV}$ and $3.3 \mathrm{keV}$ and was developed by the Swedish Institute of Space Physics (IRF)-Kiruna in Sweden, University of Bern in Switzerland and ISAS/JAXA in Japan.

Figure 2 shows the locations of the MPPE sensors on Mio. The four low-energy sensors MEA1, MEA2, MIA, and MSA are referred to as low-energy particle (LEP) sensors. The LEP sensors have ring shaped FOVs in which the center axis is perpendicular to the spin axis of the spinning Mio spacecraft. The LEP sensors are installed on the four diagonal corners of the octagonal Mio spacecraft to minimize the interference of the spacecraft body in measuring low energy charged particles. MEA1 and MEA2, the two electron sensors, and MIA and MSA, the two ion sensors, are installed $90^{\circ}$ apart to fulfill the requirements of the high time resolution measurements. The other MPPE sensors, including HEP-ion, HEPele and ENA are installed on the side panels of the Mio spacecraft. HEP-ion has a conical FOV, whereas HEP-ele and ENA have radial FOVs. To minimize the thermal input under the severe thermal conditions of Mercury's orbit, all the MPPE sensors are equipped with individual thermal shields in which the surface is coated with electrically conductive white paint.

A commonly used data processor Mission Data Processor 1 (MDP1) (Kasaba et al. 2020) controls all of the MPPE sensors and is responsible for processing the data sent from them. In addition, it formats the telemetry data, calculates the velocity moments (VMs), and reduces the quantity of data by adding, selecting, or compressing the data. Depending on the total telemetry rate of the Mio spacecraft, three different data rates of high, medium, and low are defined. The MPPE sensors are allocated to 72.5, 5.5, and $0.8 \mathrm{kbps}$ as high, medium, and low data rates, reflected by H-mode, M-mode, and L-mode, respectively. The L-mode data are continuously available during the orbital period of about $9.4 \mathrm{~h}$. The LEP sensors produce VMs of electrons and ions (density, velocity, temperature), and compressed E-t spectrograms with limited angle, mass, energy, and time resolution as L-mode data. The HEP sensors also produce count data with limited angle, mass, and energy resolution as L-mode data. ENA 
Fig. 3 View of the MEA2 sensor during vibration tests

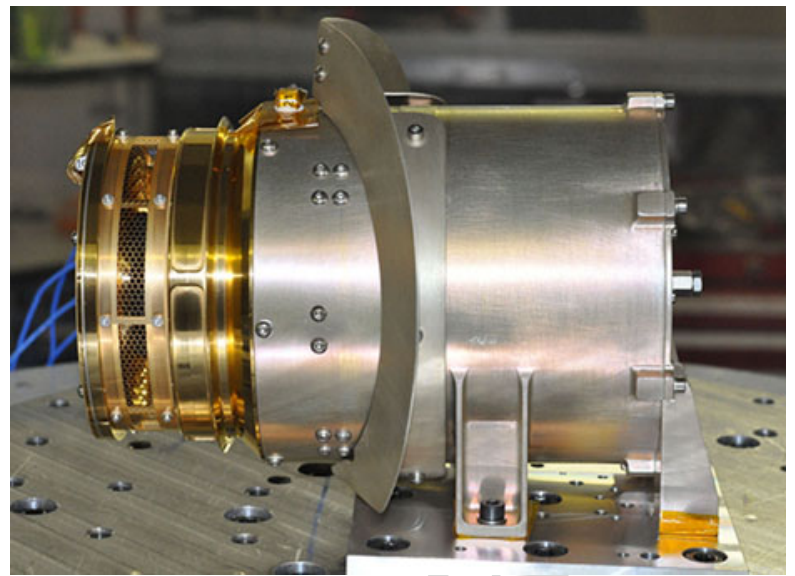

produces only L-mode data. The M-mode data are available during only about $25 \%$ of the entire observation period. The LEP and HEP sensors produce 3D counts with selected angle and time resolution or 2D counts as M-mode data. Although full 3D counts are produced as $\mathrm{H}$-mode data, this mode is available only during limited periods.

\subsection{MEA}

\subsubsection{Instrument Description of MEA}

The MEA instrument is composed of two sensors MEA1 and MEA2, which combine the selection of incoming electrons according to their energies by electrostatic deflection in symmetrical toroidal analyzers. These instruments provide a uniform angle energy response with a fast imaging particle detection system (Sauvaud et al. 2010). MEA2 is illustrated in Fig. 3. One of the key and novel features of the MEA sensors is the implementation of an electronic device that enables the geometrical factor (G-factor) to vary by a factor of 1000 in the top-hat electrostatic analyzer to measure the solar wind and magnetospheric electron fluxes within more than six decades.

Figure 4 illustrates the identical electron optic design of MEA1 and MEA2 except for the entrance aperture, which is discussed subsequently. The electrostatic analyzer (ESA) consists of a $95^{\circ}$ toroidal deflector with two concentric electrodes and a spherical top section. Whereas the outer electrode and the top-hat are at signal ground, the two parts of the inner electrode can be set at the same voltage (Uan = Utop) as for a classical top-hat analyzer with an analyzer constant $\mathrm{k}=\mathrm{E} / \mathrm{V}=9.6$. When the central part of the inner electrode (Utop) is biased with voltages lower than those applied to the toroidal part (Uan), the energy and angular acceptance are both reduced leading reduction of the G-factor.

Figure 5 shows the microchannel plate (MCP) in a chevron stack configuration, which are used to multiply the incident electrons. Figure 6 shows the 16 discrete anodes of $22.5^{\circ}$ each of which is used for position encoding that are connected to amplifiers/discriminators followed by Amptek A111F counters with a detection threshold of $10^{5}$ electrons.

Figure 7 shows the entrance of the electrostatic analyzer of MEA, which includes four baffles for reducing the penetration of ultraviolet light (UV) in the hemispherical spheres. 


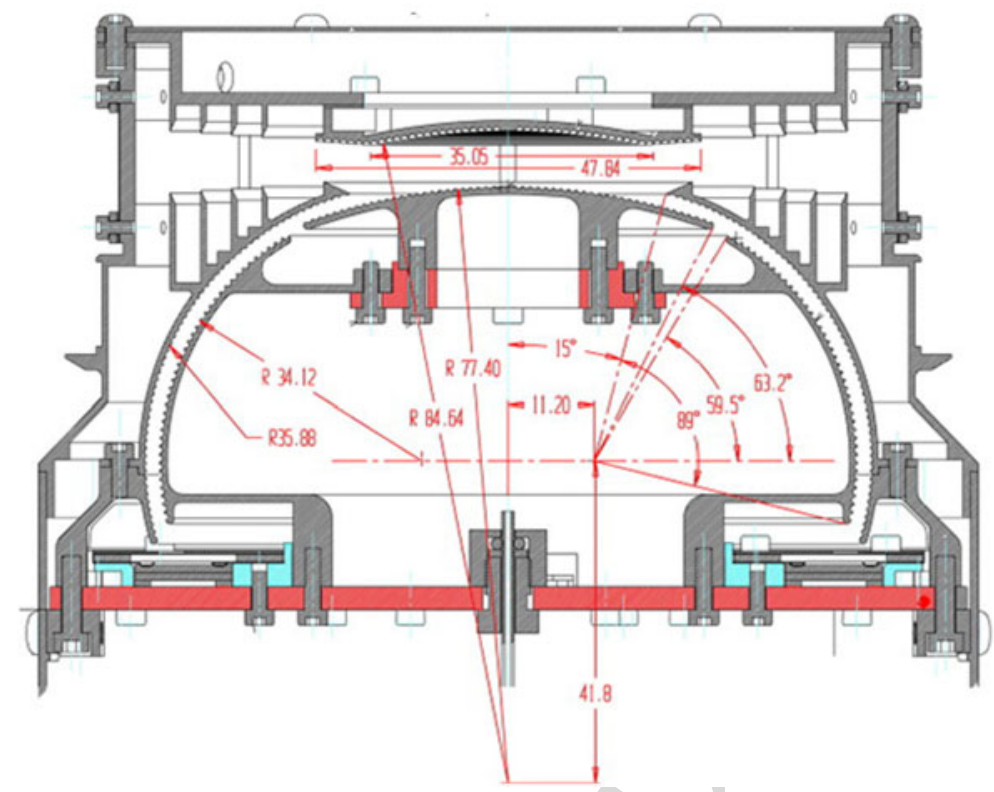

Fig. 4 Electron optic design of MEA

Fig. 5 View of the MCPs located inside the MEA2 sensor head

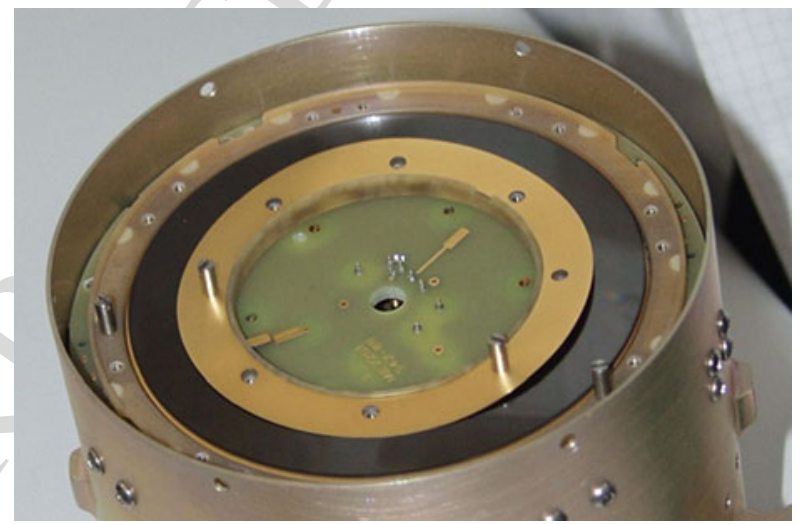

The generation of photoelectrons inside the instrument is further limited by the use of golden, polished parts that also serve to decrease the heat flux into the instrument.

Figure 8 illustrates the use of scalloping on the outer and inner plates of the toroidal deflector and the top-hat, for reducing the transmission of secondary electrons and UV photons. The analyzer plates are further coated with $\mathrm{Cu}_{2} \mathrm{~S}$ black to efficiently absorb stray light, as obtained from Collini (https://www.collini.eu).

Figure 9 shows the high voltage board which provides two sweeping voltages of 0-3000 V for the analyzer, and a static power supply from 0-3400 V for the MCPs. To select the energy of incident electrons, we vary the deflection voltages of the inner plates of the electrostatic analyzer logarithmically with 128 equally spaced steps in synchronization with the spacecraft spin period. MEA measures the full $4 \pi$ electron distributions with 
553

554

555

556

557

558

559

560

561

562

563

564

565

566

567

568

569

570

571

572

573

574

575

576

577

578

579

580

581

582

583

584

585

586

587

588

589

590

591

592

593

594

595

596

597

598

599

600
Fig. 6 Anode board of MEA2

Fig. 7 View of the entrance of the electrostatic analyzer of MEA

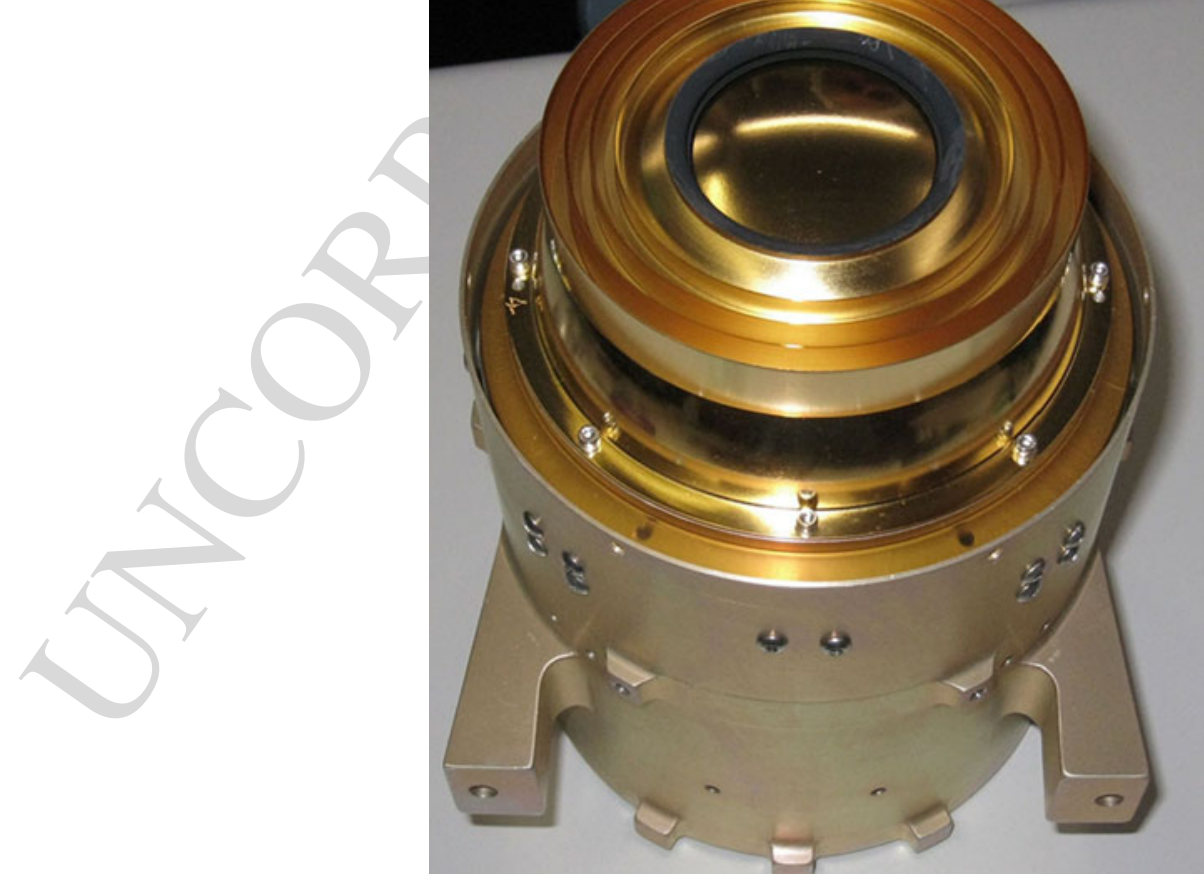

\section{基 Springer}




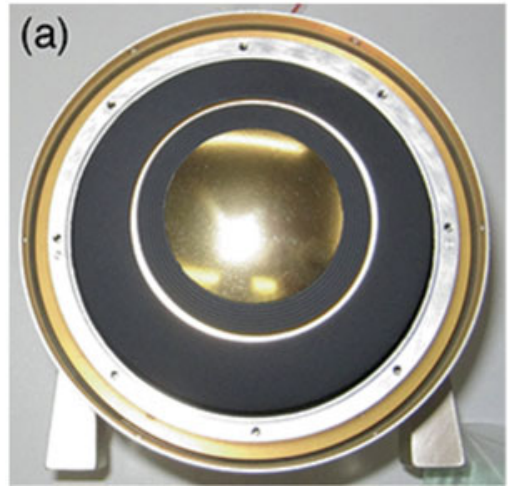

(b)

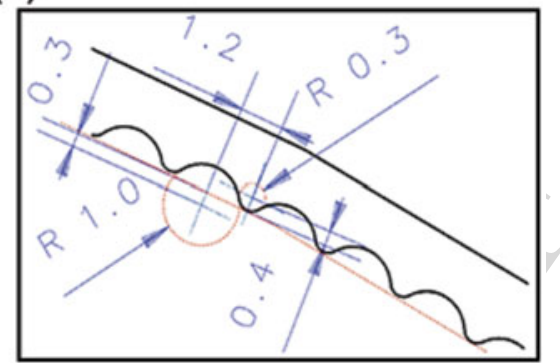

Fig. 8 (a) View of the toroidal inner sphere of MEA2. (b) Dimensions of the scalloping used

Fig. 9 High-voltage board of MEA2

a single analyzer in $1 / 2$ of the spacecraft spin period or with the two analyzers in $1 / 4$ of the period.

Figure 10 shows the field-programmable gate array (FPGA) board with two Actel RT54SX72SU components used to control all functionalities of the instrument. The first controls the sensor head and accumulates the counting rates, and the second transmits/receives data/commands from the data processing unit (MDP1) shared by all MPPE instruments using the spacewire protocol.

A multi-layer insulator (Fig. 11) and a thermal shield (Fig. 12) coated with white paint are used to ensure the thermal protection of the sensors. The peak temperatures near Mercury reach $140{ }^{\circ} \mathrm{C}$ on the thermal shield, $85{ }^{\circ} \mathrm{C}$ on the spheres, and $60{ }^{\circ} \mathrm{C}$ on the MCPs and electronic boards when operating.

Table 1 summarizes the key parameters of the MEA sensors, and Fig. 13 shows a block diagram of the instrument.

\subsubsection{Operation Mode and Data Products of MEA}

MEA includes versatile and easily programmable operating modes and data processing routines for optimizing the data collection for specific scientific studies and widely varying 
653

654

655

656

657

658

659

660

661

662

663

664

665

666

667

668

669

670

671

672

673

674

675

676

677

678

679

680

681

682

683

684

685

686

687

688

689

690

691

692

693

694

695

696

697

698

699

700
Fig. 10 FPGA board of MEA2

Fig. 11 MEA2 with its multi-layer insulator integrated with the Mio spacecraft
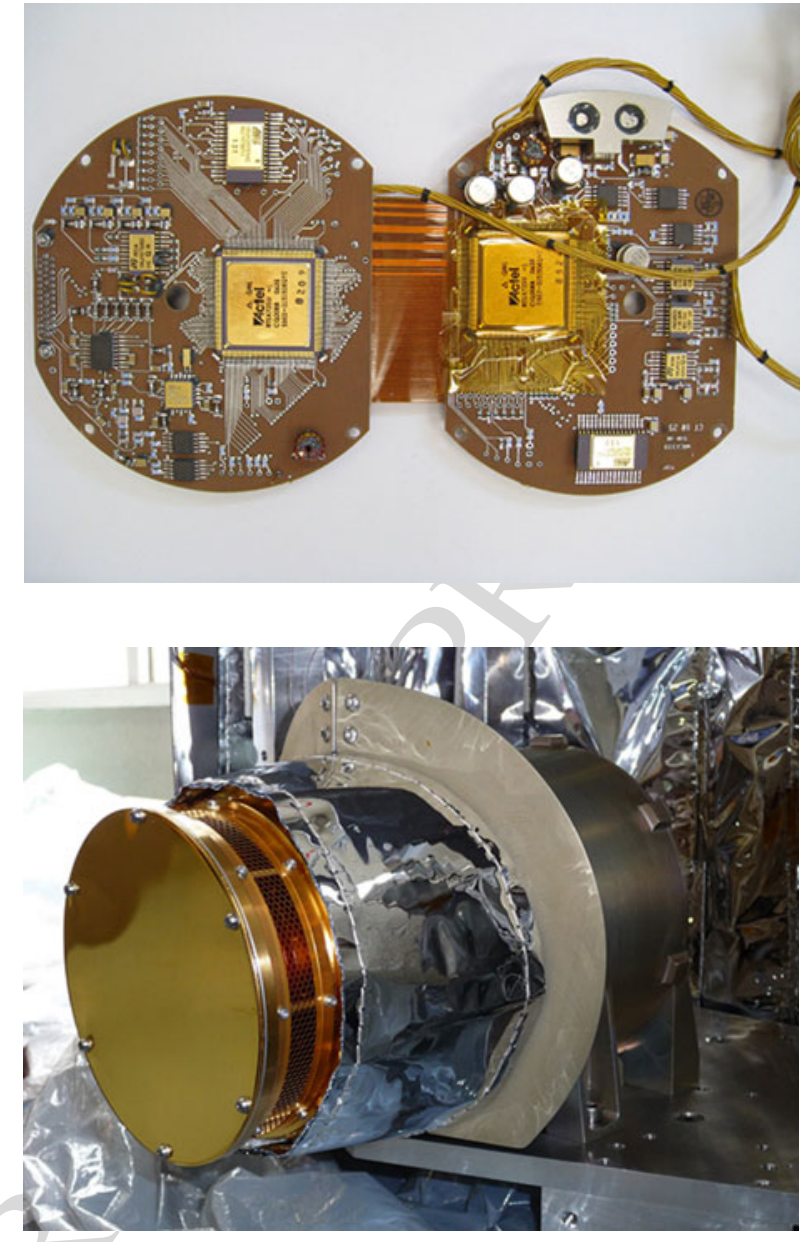

plasma regimes. Depending on the telemetry mode, MEA can transmit several MDP1 data products, including those listed below.

1. Electron omnidirectional fluxes (Et-OMN).

2. Electron VM. The instrument transmits the temperature, heat flux vector, and number density calculated in several energy bands. The position of the boundaries of each energy band is defined by commands.

3. Electron pitch angle distribution for four selected energies (Et-PAP). MEA uses the magnetic field vector as the external input for this mode and transmits the $2 \mathrm{D}$ angle-energy distribution.

4. Full 3D electron distribution. The instrument transmits a complete angular-energy spectrum accumulated for a minimum of $1 / 4$ of the spacecraft spin.

MEA uses different energy tables to adapt to the various space environment conditions encountered by the Mio spacecraft. The choice of energy table used is defined by commands. The four energy tables available for MEA include

1. $3-300 \mathrm{eV}$

글 Springer 
Fig. 12 Thermal shield of MEA2
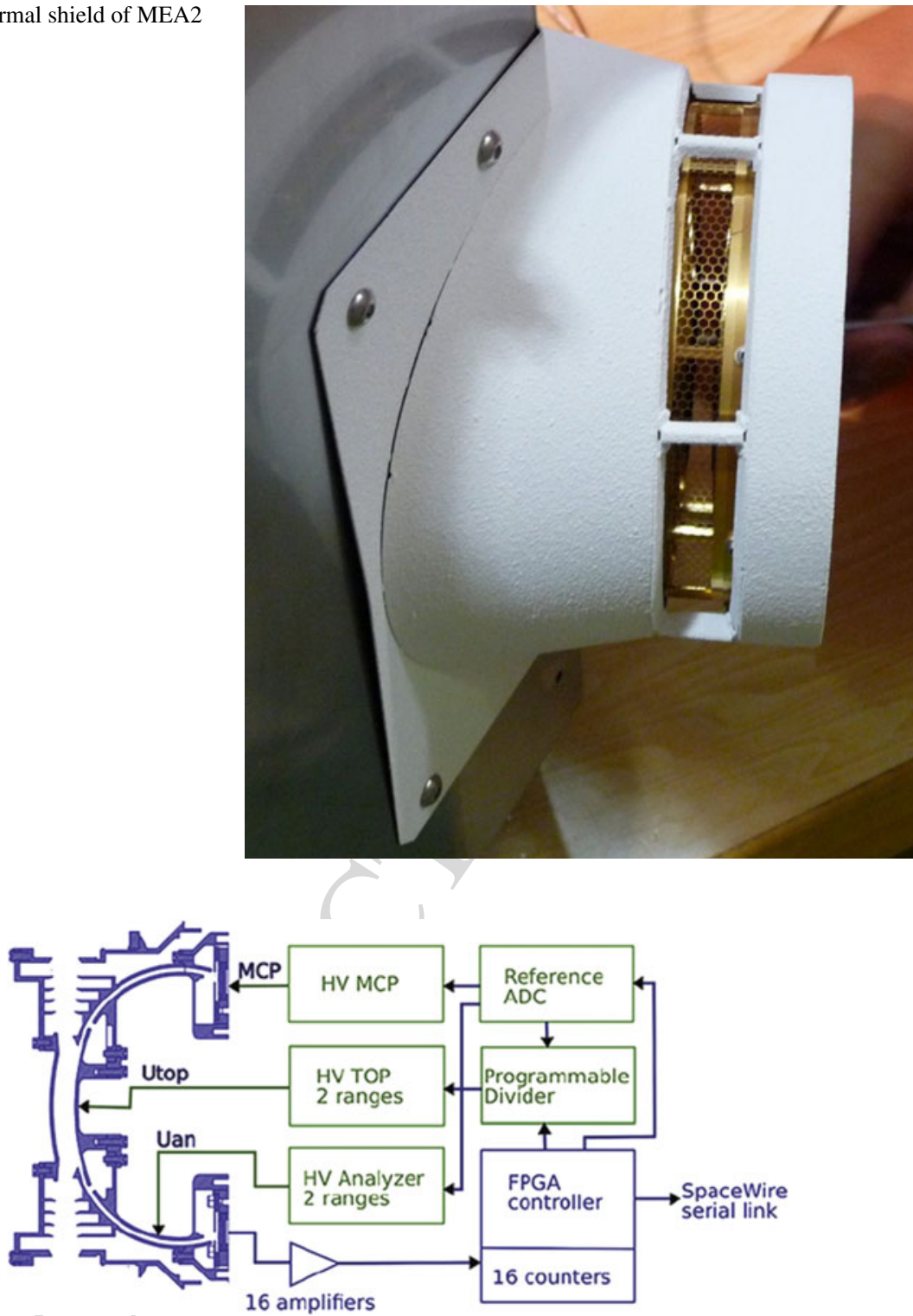

Fig. 13 Block diagram of MEA

2. $3-25000 \mathrm{eV}$

3. $3-3000 \mathrm{eV}$

4. $3000-25000 \mathrm{eV}$

In addition, MEA has unique data modes that can be defined by commands, depending on the number of channels (16 or 32) and energies (16, 32 or 64).

Table 2 shows the various MPPE data mode names and the corresponding data products of MEA1 and MEA2 together with the time resolution depending on the telemetry mode of 
Table 1 Summary of MEA performance

\begin{tabular}{|c|c|}
\hline Field of view & $8^{\circ} \times 360^{\circ}$ \\
\hline Angular resolution & $22.5^{\circ} \times 11.25^{\circ}$ \\
\hline \multirow[t]{2}{*}{ Energy range } & $3 \mathrm{eV}-25,500 \mathrm{eV}$ (Mercury mode) \\
\hline & 3 eV-3000 eV (solar wind mode) \\
\hline Energy resolution & $\Delta E / E \sim 10 \%$ (at full G-factor) \\
\hline Stepping energies and cadence & $\begin{array}{l}\text { Full energy sweep with } 64 \text { contiguous energy channels } \\
\text { every } 16 \text { or } 32 \text { times per } 4 \text { s spin }\end{array}$ \\
\hline \multirow{2}{*}{$\begin{array}{l}\text { Time resolution to obtain the full } \\
3 \mathrm{D} \text { velocity distribution function }\end{array}$} & Half a spin period, $2 \mathrm{~s}$ (using a single analyzer) \\
\hline & Quarter of a spin period, $1 \mathrm{~s}$ (using the two analyzers) \\
\hline \multicolumn{2}{|l|}{ Geometrical factor } \\
\hline MEA1 max./min. & $4.0 \times 10^{-3} / 6.7 \times 10^{-5} \mathrm{~cm}^{2} \mathrm{sreV} / \mathrm{eV}$ \\
\hline MEA2 max./min. & $2.0 \times 10^{-4} / 4.0 \times 10^{-6} \mathrm{~cm}^{2} \mathrm{sreV} / \mathrm{eV}$ \\
\hline \multirow[t]{2}{*}{ Mass } & $2.598 \mathrm{~kg}$ for MEA $1+$ MEA 2 \\
\hline & $+0.460 \mathrm{~kg}$ for their thermal shields \\
\hline \multirow[t]{3}{*}{ Power } & $2.260 \mathrm{~W}$ (average for MEA1 + MEA2) \\
\hline & $3.460 \mathrm{~W}$ (peak for MEA1 + MEA2) \\
\hline & $1.880 \mathrm{~W}$ (stand-by for MEA1 + MEA2) \\
\hline Dimensions & $177 \mathrm{~mm} \times 120 \mathrm{~mm} \phi(\mathrm{MEA} 1, \mathrm{MEA} 2)$ \\
\hline \multirow[t]{4}{*}{ Data rate } & 0.1 kbits/s (L-mdoe) \\
\hline & $2.5 \mathrm{kbits} / \mathrm{s}$ (M-mode) \\
\hline & 11 kbits/s (H-mode) \\
\hline & after factor 3 compression \\
\hline
\end{tabular}

the Mio spacecraft. Tables 3, 4, and 5 detail the properties of MEA data products for each Mio telemetry mode.

All MEA data products will be available in Common Data Format (CDF, https://cdf.gsfc. nasa.gov) files.

\subsubsection{Pre-flight Calibration of MEA}

The calibration of the two MEA sensors and a flight spare model was conducted at the IRAP Toulouse vacuum facilities. The parameters shown in Table 6 will be used to describe the various calibration setups, procedures, and results.

The pre-flight calibration of the MEA sensors consisted of full calibration of the sensors, the characterization of the MCP detectors of the sensors, and sensor testing for UV contamination. To derive the calibration parameters for a configuration as close as possible to that of the sensors in space, full calibration was performed with a realistic simulator of the instrument thermal shield and with the magnetic field of Earth inside the vacuum chamber compensated by Helmholtz coils. Figure 14 shows the MEA1 sensor installed in the setup and its simulator for the thermal shield, the beam monitor, and the magnetometer, which 
"SPAC 11214 layout: Small Condensed file: spac839.tex (karolis.kavaliauskas) class: spr-small-v1 2020/06/02 v2.07 Prn:22/07/2021; 13:21 p. 17/91» doctopic: ReviewPaper numbering style: ContentOnly reference style: aps"

MPPE on MMO (Mio)

Page 17 of 91

\#\#\#\#

801

802

803

804

805

806

807

808

809

810

811

812

813

814

815

816

817

818

81

820

821

822

823

824

825

826

827

828

82

830

831

832

833

834

835

836

837

838

839

840

841

842

843

844

845

846

847

848

849

850

Table 2 MEA data products with time resolution as a function of MPPE mode and Mio telemetry mode

\begin{tabular}{|c|c|c|c|}
\hline MPPE mode name & L-mode data products & M-mode data products & $\mathrm{H}$-mode data products \\
\hline & MEA1 & MEA1 & \\
\hline \multirow[t]{9}{*}{ 1. Default Observation Mode } & Et-OMN (4s) & Et-OMNm (4s) & N.A. \\
\hline & Et-PAP (16s) & Et-PAP (4s) & \\
\hline & VM (16s) & VM (4s) & \\
\hline & 3D-LL (640s) & 3D-M (8s) or 3D-M (4s) & \\
\hline & MEA2 & MEA2 & \\
\hline & Et-OMN (4s) & Et-OMNm (2s) & \\
\hline & Et-PAP (16s) & Et-PAP (2s) & \\
\hline & VM (16s) & $\mathrm{VM}(2 \mathrm{~s})$ & \\
\hline & MEA1 & MEA1 & MEA1 \\
\hline 2. Exospheric Mode & Et-OMN (4s) & Et-OMNm (4s) & $3 \mathrm{D}-\mathrm{H}(4 \mathrm{~s})$ \\
\hline 3. Solar Wind Mode & Et-PAP (16s) & Et-PAP (16s) & \\
\hline /IP Shock Local Mode & VM (16s) & VM (4s) & \\
\hline 4. IP Shock Macro Mode & 3D-LL (640s) & $3 \mathrm{D}-\mathrm{M}(8 \mathrm{~s})$ & \\
\hline /Bow Shock Mode & & or 3D-M (4s) & \\
\hline 5. Reconnection Mode & & & \\
\hline \multirow[t]{4}{*}{ 6. Magnetospheric Mode } & MEA2 & MEA2 & MEA2 \\
\hline & Et-OMN (4s) & Et-OMNm (2s) & $3 \mathrm{D}-\mathrm{H}(2 \mathrm{~s})$ \\
\hline & Et-PAP (4s) & Et-PAP (2s) & \\
\hline & VM (16s) & $\mathrm{VM}(2 \mathrm{~s})$ & \\
\hline
\end{tabular}

Fig. 14 MEA1 installed in the vacuum chamber.

A magnetometer is glued to the sensor housing. The instrument is turned by $90^{\circ}$ in elevation. The simulator for the thermal shield of the sensor is shown above the aperture of the sensor

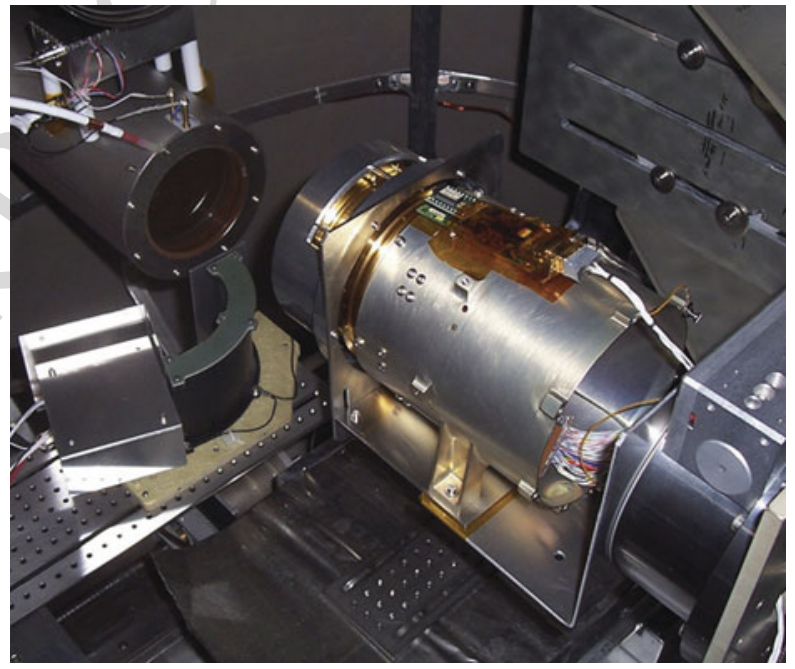

continuously measures the residual magnetic field in the vicinity of the sensor. The measurements of the magnetometer are automatically used to adjust the residual magnetic field below a maximum value of $0.5 \mu \mathrm{T}$. For each calibration step, absolute measurements of the properties of the employed electron beam were taken. 
872

873

874

875

876

Table 3 MEA1 and MEA2 data products for the low-resolution telemetry mode (L-mode)

L-mode

MEA1

\begin{tabular}{|c|c|c|c|}
\hline Data product name & Description & Time resolution (s) & Note \\
\hline Et-OMN & $\begin{array}{l}\text { E-t count data } \\
16 \text { energy }\end{array}$ & 4 & \\
\hline Et-PAP & $\begin{array}{l}\text { E-t pitch angle data } \\
4 \text { energy } \times 16 \text { pitch angle }\end{array}$ & 16 & \\
\hline VM & $\begin{array}{l}\text { velocity moment } \\
\text { n (density) } \\
\text { nVx, nVy, nVz (velocity) } \\
\text { Pxx, Pyy, Pzz } \\
\text { Pxy, Pyz, Pzx (pressure) } \\
\text { qx, qy, qz (heat flux) }\end{array}$ & 16 & $\begin{array}{l}6 \text { energy ranges } \\
0: \text { all energy steps above } \\
\text { satellite potential } * 2 ; \\
1-5: 5 \text { energy steps below } \\
\text { satellite potential } * 2\end{array}$ \\
\hline 3D-LL & $\begin{array}{l}3 \mathrm{D} \text { count data } \\
88 \text { direction } \times 16 \text { energy }\end{array}$ & 640 & \\
\hline
\end{tabular}

\section{MEA2}

\begin{tabular}{|c|c|c|c|}
\hline Data product name & Description & Time resolution (s) & Note \\
\hline Et-OMN & $\begin{array}{l}\text { E-t count data } \\
16 \text { energy }\end{array}$ & & \\
\hline Et-PAP & $\begin{array}{l}\text { E-t pitch angle data } \\
4 \text { energy } \times 16 \text { pitch angle }\end{array}$ & 16 & \\
\hline VM & $\begin{array}{l}\text { velocity moment } \\
\mathrm{n} \text { (density) }\end{array}$ & 16 & 6 energy ranges \\
\hline & $\begin{array}{l}\text { nVx, nVy, nVz (velocity) } \\
\text { Pxx, Pyy, Pzz } \\
\text { Pxy, Pyz, Pzx (pressure) } \\
\text { qx, qy, qz (heat flux) }\end{array}$ & & $\begin{array}{l}0: \text { all energy steps above } \\
\text { satellite potential } * 2 ; \\
1-5: 5 \text { energy steps below } \\
\text { satellite potential } * 2\end{array}$ \\
\hline
\end{tabular}

The counts for each MCP anode as a function of MCP HV are shown in Fig. 15. The working point of both MCP detectors for MEA1 and MEA2 was set to $2750 \mathrm{~V}$ as delineated by the red vertical line in the figure. The working point is defined here by the bias voltage applied to the MCP needed for reaching a plateau in the MCP counts.

The UV contamination test results are shown in Fig. 16. The strong count at the small energies show the photoelectrons emitted inside the instrument and the vacuum chamber. The maximal background was less than 1 per second per anode.

Full calibration of the MEA1 and MEA2 sensors was conducted in the coordinate frame shown in Fig. 17. The electron beam properties are given below.

- For each azimuth angle $\Phi$, a scan was made over elevation angle $\Theta$.

- For each angular position, the set of G-factors was tested.

- For each value of G-factor, the analyzer voltage was scanned.

Figures 18 and 19 show the energy, elevation and azimuthal responses for different anodes and G-factor levels. The dashed curves in these plots show the polynomial fit that 
"SPAC 11214 layout: Small Condensed file: spac839.tex (karolis.kavaliauskas) class: spr-small-v1 2020/06/02 v2.07 Prn:22/07/2021; 13:21 p. 19/91" doctopic: ReviewPaper numbering style: ContentOnly reference style: aps"

MPPE on MMO (Mio)

Page 19 of 91

\#\#\#\#-

903

90

905

906

907

908

909

910

911

912

913

914

915

916

917

918

919

920

921

922

923

924

925

926

927

928

929

930

931

932

933

934

935

936

937

938

939

940

941

942

943

944

945

946

947

948

949

950

Table 4 MEA1 and MEA2 data products for the medium-resolution telemetry mode (M-mode)

M-mode

MEA1

Data product name Description Time resolution (s) Note

\begin{tabular}{|c|c|c|c|}
\hline Et-OMNm & $\begin{array}{l}\text { E-t count data } \\
32 \text { energy }\end{array}$ & 4 & \\
\hline Et-PAP & $\begin{array}{l}\text { E-t pitch angle data } \\
4 \text { energy } \times 16 \text { pitch angle }\end{array}$ & 4 & $\begin{array}{l}4 \text { starting energy steps } \\
\text { and width are selectable by commanding }\end{array}$ \\
\hline VM & $\begin{array}{l}\text { velocity moment } \\
\mathrm{n} \text { (density) } \\
\mathrm{nVx}, \mathrm{nVy}, \mathrm{nVz} \text { (velocity) } \\
\text { Pxx, Pyy, Pzz } \\
\text { Pxy, Pyz, Pzx (pressure) } \\
\text { qx, qy, qz (heat flux) }\end{array}$ & 4 & $\begin{array}{l}6 \text { energy ranges } \\
0: \text { all energy steps above } \\
\text { satellite potential } * 2 \\
1-5: 5 \text { energy steps below } \\
\text { satellite potential } * 2\end{array}$ \\
\hline 3D-M (8s) & $\begin{array}{l}\text { 3D count data } \\
88 \text { direction } \times 16 \text { energy }\end{array}$ & 8 & $($ MPPE mode $=01235$ ) \\
\hline 3D-M (4s) & $\begin{array}{l}3 \mathrm{D} \text { count data } \\
88 \text { direction } \times 16 \text { energy }\end{array}$ & 4 & $($ MPPE mode $=4678)$ \\
\hline
\end{tabular}

MEA2

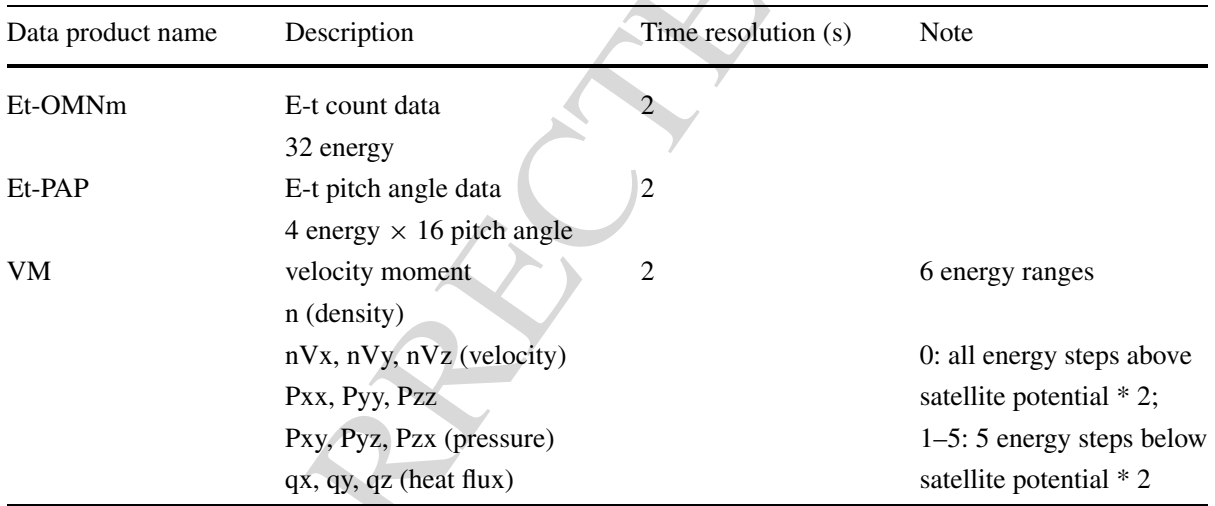
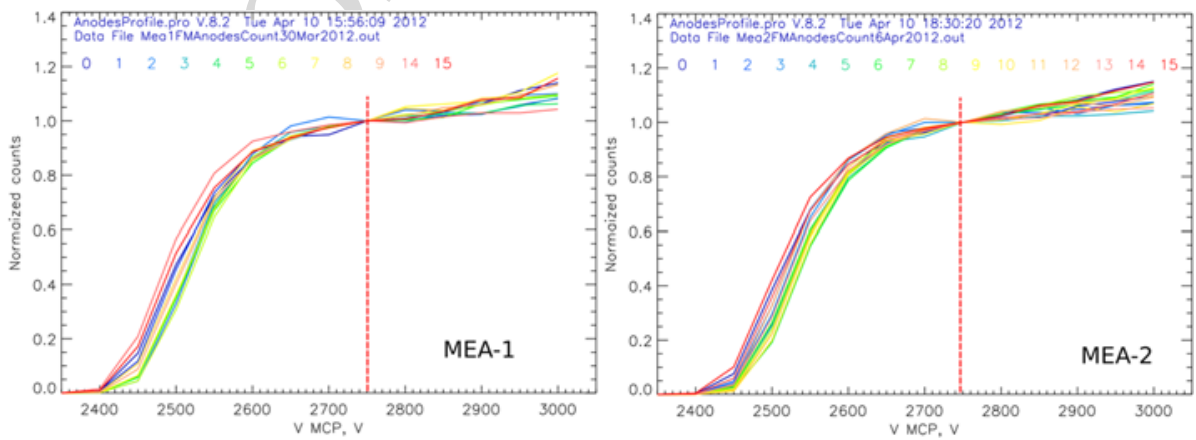

Fig. 15 MCP counts as a function of MCP bias voltage. Each of the 16 anodes is represented by a different color, with the anode number printed in the same color at the top of the plot 
"SPAC 11214 layout: Small Condensed file: spac839.tex (karolis.kavaliauskas) class: spr-small-v1 2020/06/02 v2.07 Prn:22/07/2021; 13:21 p. 20/91" doctopic: ReviewPaper numbering style: ContentOnly reference style: aps"

_\#\#\#_ Page 20 of 91

Y. Saito et al.

953

954

955

956

957

958

959

960

961

962

963

964

965

966

967

968

969

970

971

972

973

974

975

976

977

978

979

980

981

982

983

984

985

986

987

988

989

990

991

992

993

994

995

996

997

998

999

1000

Table 5 MEA1 and MEA2 data products for the high-resolution telemetry mode (H-mode)

\begin{tabular}{llll}
\hline H-mode & & & \\
\hline MEA1 & & Time resolution (s) & Note \\
\hline $\begin{array}{l}\text { Data product name } \\
\text { 3D-H }\end{array}$ & Description & 4 & 16 sectors, 8 channels \\
& 3D count data & & \\
\hline MEA2 & Description & Time resolution (s) & Note \\
\hline Data product name $\times 32$ energy & 2D count data & & 16 sectors, 8 channels + \\
\hline 3D-H & 88 direction $\times 32$ energy $\times 2$ & & 16 sectors, 8 channels \\
\hline
\end{tabular}
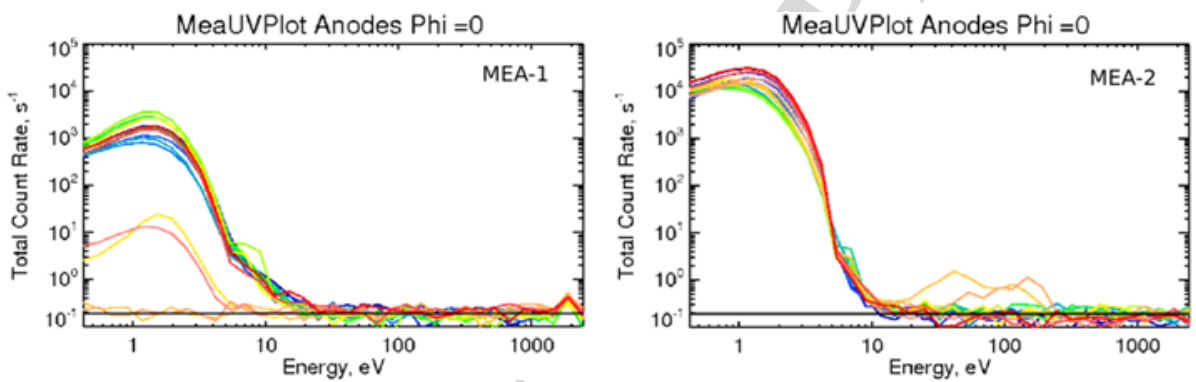

Fig. 16 UV contamination test results for MEA1 and MEA2. Each of the 16 anodes is represented by a different color as described in Fig. 15

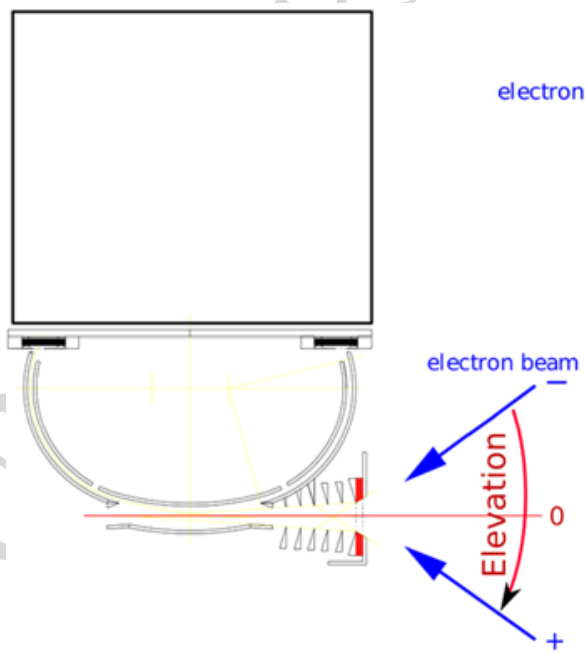

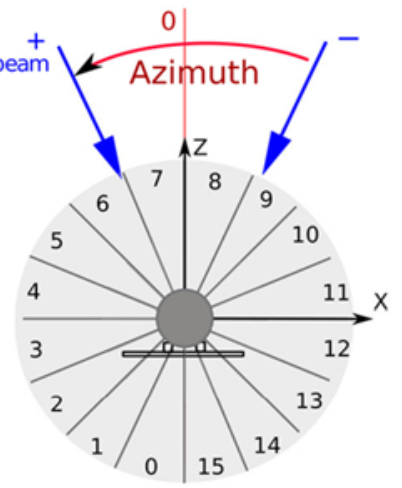

View from the top of the sensor head

Fig. 17 Definition of the elevation and azimuth angles for full calibration of the MEA sensors

Springer

Journal ID: 11214, Article ID: 839, Date: 2021-07-22, Proof No: 2, UNCORRECTED PROOF 


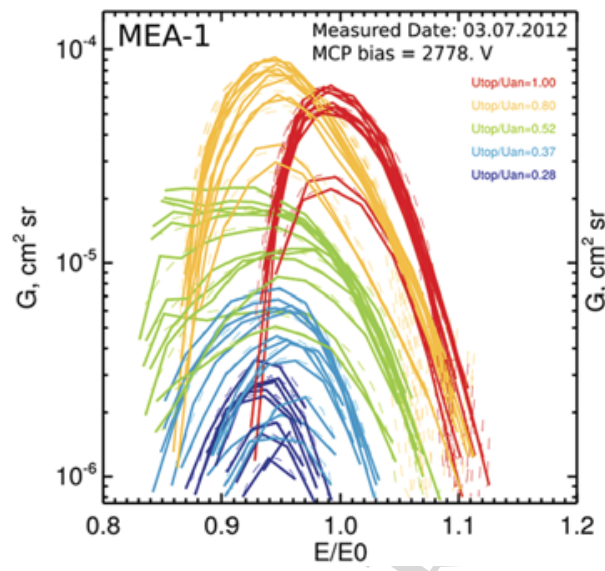

Incident electron energy, eV

Elevation angle

Azimuth angle

Analyzer voltage

Top part of analyzer voltage

$\begin{array}{ll}\text { K } & \mathrm{E}=\mathrm{Uan} \cdot \mathrm{K} \\ \mathrm{K} 0 & \text { Best K for the current } \Theta \text { and } \Phi\end{array}$

$\triangle \mathrm{E} / \mathrm{E} \quad$ Energy resolution of the analyzer

$\mathrm{P}_{\mathrm{BEAM}} \quad$ Electron beam flux $\mathrm{cm}^{-2} \mathrm{~s}^{-1}$ as a function of the elevation angle

$\Omega_{i} \quad$ One azimuthal sector aperture, $\mathrm{cm}^{2}$ for fixed $\Theta, \Phi$, Uan and Utop

$\mathrm{Ci} \quad$ Count rate, $\mathrm{s}^{-1}$ of one azimuthal sector

Gi One sector G-factor, $\mathrm{cm}^{2} \mathrm{sreV} / \mathrm{eV}$

G Total G-factor of the instrument, $\mathrm{cm}^{2} \mathrm{sreV} / \mathrm{eV}$ (used for numerical simulation) $\mathrm{HV}_{\mathrm{MCP}} \quad \mathrm{MCP}$ high voltage, $\mathrm{V}$, measured at the HV unit level

Fig. 18 Energy responses of different anodes for various G-factor levels. Each of the 16 anodes is represented for each G-factor level

enabled definition of $\Delta E / E$ and $\Delta \Omega$ with high accuracy. When the central part of the inner electrode (Utop) is biased with voltages lower than those applied to the toroidal part (Uan), the analyzer accepts particles coming from a slightly higher azimuth. The energy and angular acceptance are both reduced leading to a reduction of the G-factor. The G-factor for each anode versus the value of Utop/Uan is shown in Fig. 20, where the theoretical profile obtained from the numerical simulation is represented by a dashed curve. MEA2 has a maximum GF0/GF ratio of 1000, whereas that of MEA1 is only 60 because it includes a grid attenuator with 5\% transparency at its entrance. Table 7 summarizes the calibration results for MEA1 and MEA2.

\subsubsection{Near-Earth Commissioning Results of MEA}

On July 1 and 2, 2019, the two MEA sensors were turned on, respectively, when the BepiColombo spacecraft was about 29 million $\mathrm{km}$ from Earth. MEA1 and MEA2 have per- 

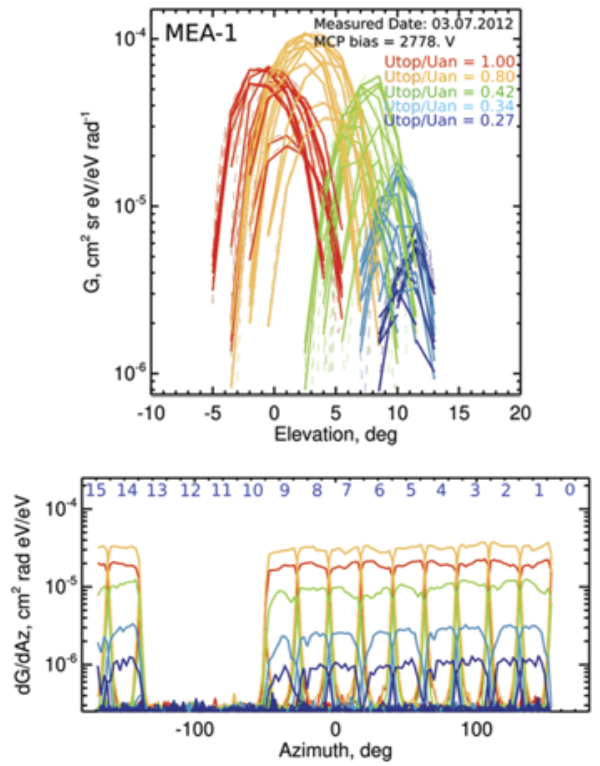
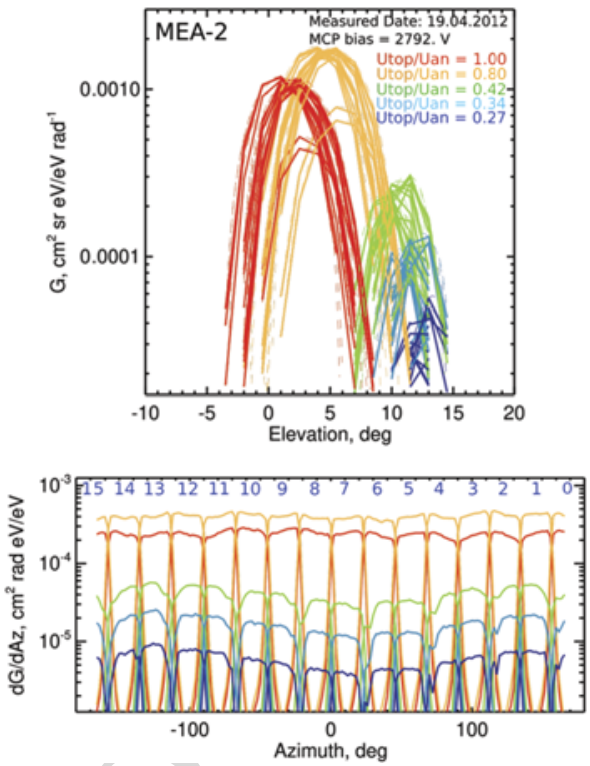

Fig. 19 Top: Elevation response of the 16 anodes for various G-factor levels (in color). Each of the 16 anodes is represented for each G-factor level. Bottom: azimuthal response of the 16 anodes for various G-factor levels (in color, same color code as used on the top panel)
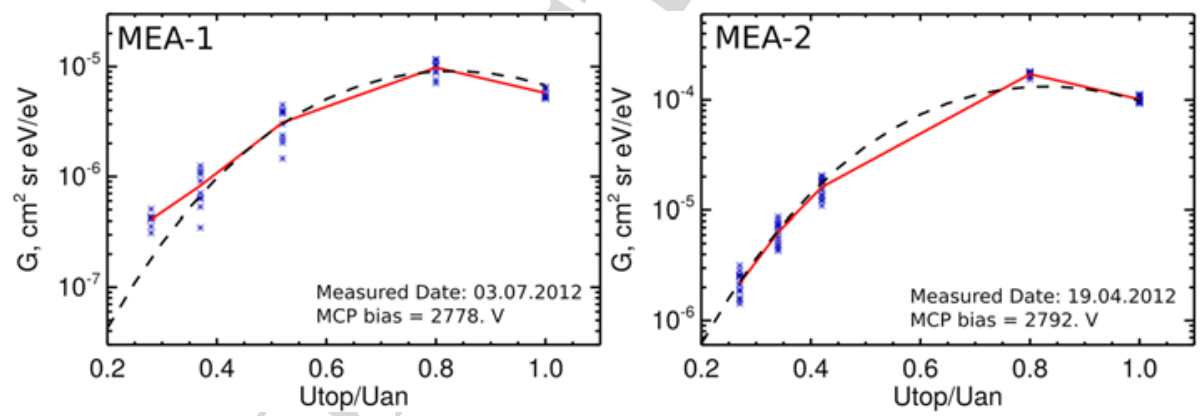

Fig. 20 Anodes G-factors as a functions of Utop/Uan. The dashed lines show the theoretical profile scaled with the appropriate factor

fectly responded to our commands up to their nominal working point of $2750 \mathrm{~V}$ applied to their MCPs. Hence, the very first electron spectra in the solar wind have been successfully obtained, even though Mio is behind the MOSIF thermal shield. Figures 21 and 22 show the MEA1 and MEA2 data, respectively. The solar wind electron moments were estimated from MEA1 3D data after noise removal (Fig. 23). The density calculated from the MEA1 data when integrated over all energies, including both core and halo (above $100 \mathrm{eV}$ ) solar wind electrons $\left(1.9 \mathrm{~cm}^{-3}\right)$, and the first eleven energies for the core solar wind electrons $\left(0.9 \mathrm{~cm}^{-3}\right)$, and the temperature $(13 \mathrm{eV})$, agree well with expected values at the location 
Table 7 Summary of MEA1 (top) and MEA2 (bottom) calibration. GF corresponds to the geometrical factor of the instrument $\left(\mathrm{cm}^{2} \mathrm{sreV} / \mathrm{eV}\right)$, and $\mathrm{GF} 0$ corresponds to the GF when Utop $=$ Uan. $\Delta \theta$ is the elevation FWHM. The remaining parameters are defined in Table 6

\begin{tabular}{|c|c|c|c|c|}
\hline $\mathrm{K}$ & 8.67 & 8.48 & 8.57 & 8.51 \\
\hline$\Delta \mathrm{E} / \mathrm{E}$ & 0.11 & 0.16 & 0.11 & 0.09 \\
\hline \multicolumn{5}{|l|}{ MEA2 } \\
\hline Utop/Uan & 0.8 & 0.52 & 0.37 & 0.28 \\
\hline $\mathrm{GF} \mathrm{cm}^{2} \mathrm{sreV} / \mathrm{eV}$ & $2 \mathrm{e}-4$ & $6.7 e-5$ & $1.6 \mathrm{e}-5$ & $4.0 \mathrm{e}-6$ \\
\hline GF0/GF & 20 & 60 & 250 & 1000 \\
\hline$\Theta \operatorname{deg}$ & 1.8 & 6.3 & 7.5 & 9.0 \\
\hline$\Delta \Theta \operatorname{deg}$ & 5.9 & 5.0 & 3.0 & 3.5 \\
\hline K & 8.67 & 8.62 & 8.57 & 8.51 \\
\hline$\Delta \mathrm{E} / \mathrm{E}$ & 0.11 & 0.13 & 0.11 & 0.08 \\
\hline
\end{tabular}

\section{MEA1}

\begin{tabular}{lllll} 
Utop/Uan & 0.8 & 0.42 & 0.34 & 0.27 \\
$\mathrm{GF} \mathrm{cm}^{2}$ sreV/eV & $4 \mathrm{e}-3$ & $6.7 \mathrm{e}-3$ & $2.0 \mathrm{e}-3$ & $6.7 \mathrm{e}-5$ \\
$\mathrm{GF} 0 / \mathrm{GF}$ & 1 & 6 & 20 & 60 \\
$\Theta \mathrm{deg}$ & 1.8 & 7.0 & 8.2 & 9.0 \\
$\Delta \Theta \mathrm{deg}$ & 5.9 & 4.5 & 3.0 & 3.6 \\
$\mathrm{~K}$ & 8.67 & 8.48 & 8.57 & 8.51 \\
$\Delta \mathrm{E} / \mathrm{E}$ & 0.11 & 0.16 & 0.11 & 0.09 \\
\hline
\end{tabular}

Produced by CLWeb

Fig. 21 First MEA1 data obtained in the solar wind on July 1, 2019. The first panel is the GF of the sensor that was varied by decreasing the ratio Utop/Uan from 1 to its lowest value at the end of the interval (for corresponding values of the GF see Fig. 20 from right to left). The vertical lines delineate the commands sent to MEA1 during the time period, particularly when the HV was raised to nominal values of $2750 \mathrm{~V}$. The three following panels indicate the energy-time spectrogram of Et-OMN data (in counts), 3D data, and pitch-angle distributions for four selected energies, in L-mode 
Fig. 23 MEA1 moment (density, temperature) estimated from 3D data (blue) and compared with a Maxwellian distribution function (red), after noise removal. The spike observed above $100 \mathrm{eV}$ corresponds to part of the halo solar wind electron distribution function

of the Mio spacecraft as well as those with a Maxwellian distribution with a density of $0.6 \mathrm{~cm}^{-3}$ and temperature of $10 \mathrm{eV}$.

The very first data obtained during the near-Earth orbit phase commissioning of the MEA instrument confirm that both MEA1 and ME2 are working normally. In the near future the MEA instrument will be turned on again during Earth, Venus, and Mercury fly-bys 
Fig. 24 MIA flight model delivered to the Mio system in June 2014

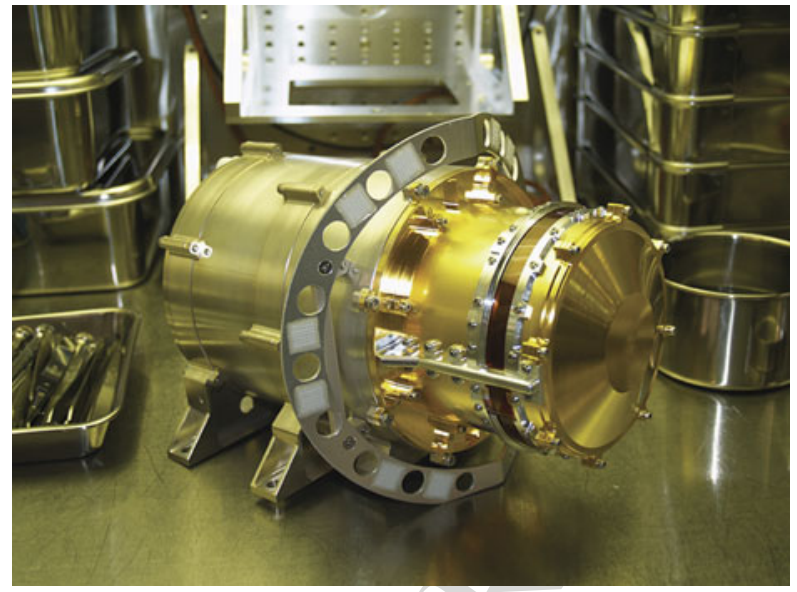

and during the cruise phase to enable multipoint measurements of electrons in the solar wind together with electron spectrometers onboard the Solar Orbiter and Parker Solar Probe missions.

\subsection{MIA}

\subsubsection{Instrument Description of MIA}

MIA as shown in Fig. 24 was developed to understand the structure and plasma dynamics of Mercury's magnetosphere; Mercury-solar wind interaction; atmospheric abundances, structures, and generation/loss processes; and the solar wind between 0.3 and 0.47 AU (Miyake et al. 2009). To achieve these research objectives, MIA should be able to measure both the $3 \mathrm{D}$ distribution function of solar wind ions around Mercury (0.3-0.47 AU) and the planet's magnetospheric ions. Figure 25 shows a block diagram of MIA, which consists of the (A) spacecraft interface board, (B) positive high voltage board, (C) negative high voltage board, and (D) analyzer. As shown in Fig. 25(D), MIA is a top-hat type electrostatic analyzer with toroidal deflectors (Saito et al. 2010a). Figure 26 shows the "top-cap" and upper part of the entrance collimator (panel (a)) and inner sphere and lower part of the entrance collimator (panel (b)). The surface of the analyzer is gold plated or blackened by copper sulfide black. The blackening process "Ultraviolet Absorbing Black plating" was developed by Mitsuya Co. Ltd. in Japan (https://www.mitsuya-plating.com). In addition, the inner and outer spheres are serrated with the tip-to-root length and tip angle of the sawtooth serrations at $0.5 \mathrm{~mm}$ and $60^{\circ}$, and the light traps are placed at the top part of the outer sphere to minimize the solar UV entering the detector (MCP). MIA measures 3D ion distribution function utilizing the spin motion of the spacecraft. The diameters of the inner and outer toroidal electrodes are $32 \mathrm{~mm}$ and $35 \mathrm{~mm}$, respectively, with the center shifted $5 \mathrm{~mm}$ toward the radial direction. The resultant analyzer constant is 5.66 .

Stepping high voltage between $0 \mathrm{~V}$ and $-5 \mathrm{kV}$ is applied to the inner toroidal electrode. Figure 27 shows the spacecraft interface board. On the rear side (panel (a)), two MDM connectors are shown including a 9-pin checkout connector and a 25-pin SpaceWire/power supply interface connector. On the front side (panel (b)), two Hypertac connectors are placed including one connected to the high voltage boards and the other connected to the application specific integrated circuit (ASIC) on the MCP anode. Figure 28 shows the negative 
Fig. 25 MIA block diagram
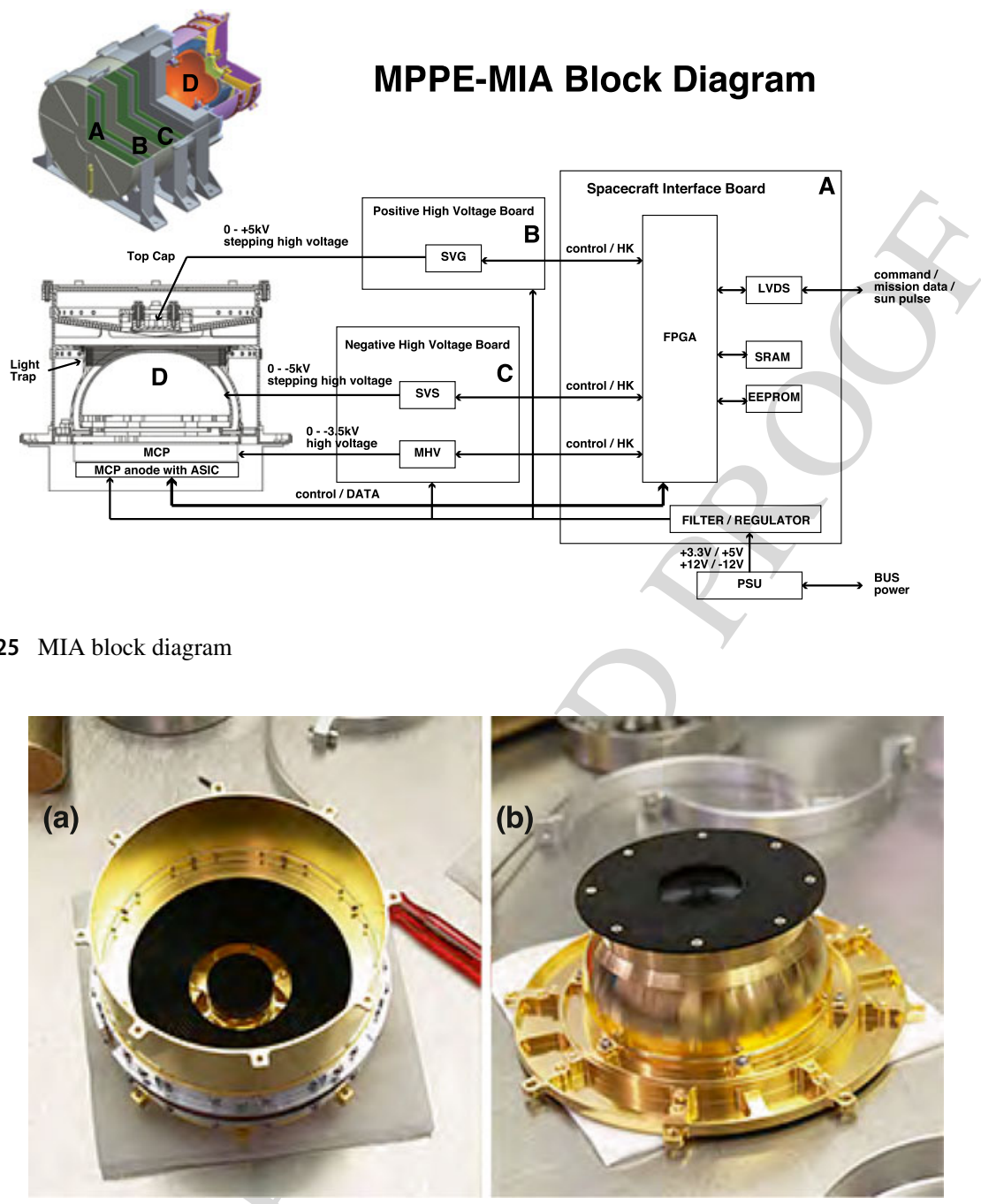

Fig. 26 (a) "Top-cap" and upper part of the entrance collimator of MIA. (b) Inner sphere and bottom part of the entrance collimator of MIA. The parts are gold plated or blackened by copper sulfide black

high voltage board installed in the chassis of MIA (Fig. 25(C)). Among the three electronics boards (Fig. 25(A)-(C)) shielding plates are installed to reduce the electrical noise and the risk of electrical discharge among the electronics boards. Ions enter the analyzer by passing through the collimator and are attracted down toward the inner electrode by receiving Coulomb force from the electric field between the "top-cap" and the inner electrode. Ions with specific energy ranges determined by the high voltage applied to the inner electrode can pass through the toroidal analyzer, enter the Z-stack MCP and become multiplied to generate detectable amounts of charge clouds. A grid having the same voltage as the input surface of the MCP is placed between the MCP and the toroidal analyzer.

The charge clouds from the MCP are detected by a 63-channel discrete anode (Saito et al. 2017). The incident azimuthal directions of the ions correspond to the positions at which 

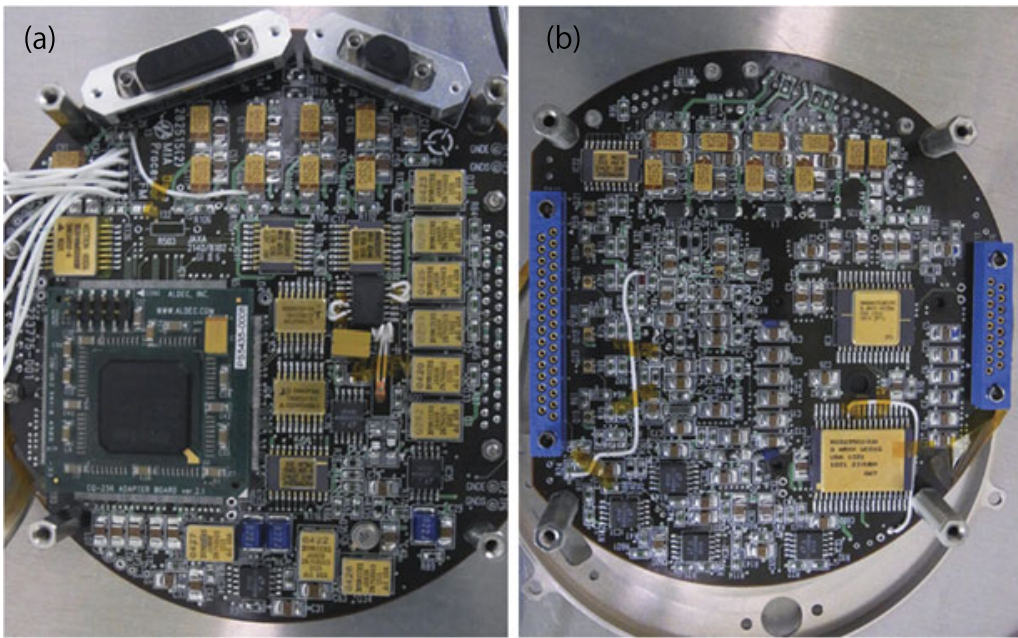

Fig. 27 Digital interface board of MIA. (a) Rear side, (b) front side

Fig. 28 Negative high voltage board installed in the chassis of MIA. A Hypertac connector from ASIC on the MCP anode is also shown

\section{the charge clouds are detected. The detected charge clouds are fed into a newly developed} ASIC of which the bare chip is installed at the back side of the discrete MCP anode. The ASIC consists of 64-channel discriminators, 64-channel fast preamplifiers, and 64-channel counters (Saito et al. 2017).

MIA should measure both intense solar wind ions and tenuous Mercury magnetospheric ions. Therefore, the required dynamic range for detecting low-energy ion flux is as wide as $10^{6}$ (Mukai et al. 2004). To measure both solar wind ions without saturation and Mercury magnetospheric ions with sufficient counting statistics, MIA includes an attenuation grid with $10 \%$ transmission placed at limited channels (one of the two $\pm 60^{\circ}$ angular ranges 
Fig. 29 Attenuation grid of MIA. (a) The relationship between the spin axis and the entrance of MIA with the mechanical attenuation grid. (b) Ion entrance of MIA with the mechanical attenuation grid. (c) Pattern of attenuation grid

Fig. 30 MIA with thermal shield installed on one of the eight corners of the Mio spacecraft. The entrance aperture is covered by a Kapton sheet that was removed before the launch

centered at the spin plane) of the entrance part of the analyzer. Figure 29 shows a schematic diagram of the attenuation grid pattern.

In addition, MIA includes a function for reducing the geometrical factor electrically for solar wind ion measurement. The sensitivity of the analyzer can be reduced by applying positive high voltage to the "top-cap" insulated from the surrounding structures. By applying stepping high voltage between $0 \mathrm{~V}$ and $+5 \mathrm{kV}$ and synchronizing with the inner sphere voltage, the G-factor can be reduced to $\sim 1 / 50$ (Miyake et al. 2009).

To reduce the strong thermal input to MIA on Mercury orbit, MIA is equipped with its own thermal shield (Fig. 30). The thermal shield is composed of titanium and the surface is painted with electrically conductive white paint.

According to our knowledge of Earth's magnetosphere, full 3D measurements of lowenergy ions with high time resolution are indispensable for understanding the structure and dynamics of the magnetosphere. Because no full 3D low-energy ion data have been obtained 
around Mercury, the low-energy ion data obtained by MIA together with MSA on Mio will provide unique opportunity for understanding the detailed structure and dynamics of the Mercury magnetosphere.

\subsubsection{Operation Mode and Data Products of MIA}

After its insertion into Mercury's orbit, MIA will continue its observation during all orbital phases except for periods in which MIA should be turned off owing to thermal/power constraints. Because the operation of all science instruments on Mio should be synchronized, the scientific operation of MIA obeys that of the Mio instrument suite. MIA has three operational modes: solar wind (SW), magnetospheric ion high angular resolution (MIHAR), and magnetospheric ion low angular resolution (MILAR) modes. The SW mode is used for fine angular resolution measurement of the solar wind around Mercury; the MIHAR mode is used for high angular resolution measurements of the Mercury magnetospheric ions; and the MILAR mode is used for low angular resolution measurements of the Mercury magnetospheric ions. These modes are changed depending on the satellite position and telemetry data rate by real-time commanding or stored commands. The MDP1 onboard software can also be used to change the mode. Table 8 shows the MIA operation mode and the data rate sent from MIA to MDP1. MIA always acquires data with a fixed sampling time of $\sim 2 \mathrm{~ms}$, a fixed spin angular sector of $5.625^{\circ}$ (64 equally divided spin sectors: $360^{\circ} / 64$ sectors $=5.625^{\circ}$ ), and 64 ASIC channels. The 64 ASIC channels are connected to the 62-channel discrete anode that detects the position of the energy analyzed ions and an annular anode that is used for monitoring the high-energy particle background (Saito et al. 2017). One ASIC channel is left open to monitor the electrical background noise (Fig. 36). Therefore MIA always acquires 64 channels $\times 64$ spin sectors $\times 32$ energy steps $=131072$ data for 1 spin.

Since this data quantity is too large for processing by MDP1, the FPGA in MIA will add adjacent counts (spin sectors and ASIC channels) depending on the MIA's data mode (modes 0,1 and 2). The data sent from MIA to MDP1 are processed by MDP1 and the telemetry data are transmitted to the ground according to the MPPE data mode described in Sect. 4.

Table 9 shows six different MIA energy sweep modes. Mode 0 and 1 are used mainly for solar wind ion observation. The energy range between $\sim 100 \mathrm{eV} / \mathrm{q}$ and $\sim 10 \mathrm{keV} / \mathrm{q}$ is exponentially divided into 128 energy steps. To cover the full energy range, four spin-periods (nominally $16 \mathrm{~s}$ ) are necessary. The difference between energy sweep modes 0 and 1 is that the sensitivity control function is either OFF or ON. For mode 1, the sensitivity is controlled by applying positive high voltage to the "top-cap" to reduce the G-factor. Energy sweep mode 2 is referred to as the MCP protection mode, which is used for protecting part of the MCP that detects ions from the analyzer azimuthal sector with no mechanical attenuation grid. The energy range is determined not to measure intense main component of the solar wind. Energy sweep modes 3, 4, and 5 are used mainly for magnetospheric ion observation. Energy sweep mode 3 is a "wide energy range mode" that covers the full energy range between $\sim 20 \mathrm{eV} / \mathrm{q}$ and $\sim 25 \mathrm{keV} / \mathrm{q}$ with 32 exponentially divided steps. Energy sweep mode 4 is a low energy range mode that covers the low energy range between $\sim 20 \mathrm{eV} / \mathrm{q}$ and $\sim 5 \mathrm{keV} / \mathrm{q}$ with 32 exponentially divided steps. Energy sweep mode 5 is a high energy range mode that covers the high-energy range between $\sim 5 \mathrm{keV} / \mathrm{q}$ and $\sim 25 \mathrm{keV} / \mathrm{q}$ with 32 exponentially divided steps. Different energy sweep modes can be selected for eight spin sector groups, where spin sector group 0 is from spin sector 0 to 7 , group 1 is from spin sector 8 to $15, \ldots$, and group 7 is from spin sector 56 to 63 . Spin sector 0 occurs when the axis of rotational symmetry of MIA is pointing away from the Sun (Fig. 31). In this case, the 
Table 8 MIA operation mode

\begin{tabular}{|c|c|c|}
\hline Mode & Produced data & Raw data rate \\
\hline $\begin{array}{l}\text { Solar Wind Mode } \\
\text { (DATA MODE 1) }\end{array}$ & $\begin{array}{l}\text { Count data } \\
\text { 1) }\left(8+8^{* 1}\left(22.5^{\circ}\right) \text { polar sectors }\right. \\
+2 \text { background counters }) \\
\times 8\left(22.5^{\circ}\right) \text { equatorial sectors } \\
\times 32 \text { energy steps/spin } \\
2)\left(8+8^{* 1}\left(22.5^{\circ}\right) \text { polar sectors }\right. \\
+2 \text { background counters }) \\
\times 8\left(11.25^{\circ}\right) \text { equatorial sectors } \\
\times 32 \text { energy steps/spin } \\
3)\left(8+4^{* 2}\left(22.5^{\circ}\right) \text { polar sectors }\right. \\
+2 \text { background counters }) \\
\times 16\left(5.625^{\circ}\right) \text { equatorial sectors } \\
\times 32 \text { energy steps } / \text { spin } \\
(\text { excluding } 90 \text { deg. } \times 90 \text { deg. sol } \\
4) 16 \text { polar sectors } \times 16 \text { equatori } \\
\times 128 \text { energy steps } / 4 \text { spins } \\
(90 \text { deg. } \times 90 \text { deg. solar wind se }\end{array}$ & $\begin{array}{l}\text { Max. } 245760 \mathrm{bps} \\
\text { Average } 98304 \mathrm{bps}\end{array}$ \\
\hline $\begin{array}{l}\text { Magnetospheric Ion } \\
\text { High Angular } \\
\text { Resolution Mode } \\
\text { (DATA MODE 2) }\end{array}$ & $\begin{array}{l}\text { count data } \\
\left(16+16^{* 3} \text { polar sectors }\right. \\
+2 \text { background counters }) \\
\times 32 \text { equatorial sectors } \\
\times 32 \text { energy steps/spin }\end{array}$ & $\begin{array}{l}139264 \text { bps } \\
16 \text { bits/data }\end{array}$ \\
\hline $\begin{array}{l}\text { Magnetospheric Ion } \\
\text { Low Angular } \\
\text { Resolution Mode } \\
\text { (DATA MODE 3) }\end{array}$ & $\begin{array}{l}\text { count data } \\
\left(8+8^{* 1} \text { polar sectors }\right. \\
+2 \text { background counters }) \\
\times 16 \text { equatorial sectors } \\
\times 32 \text { energy steps/spin }\end{array}$ & $\begin{array}{l}36864 \text { bps } \\
16 \text { bits/data }\end{array}$ \\
\hline
\end{tabular}

$* 1$ Sensitivity of about $120^{\circ}$ in the eight polar sectors is reduced down to $1 / 50$ with mechanical attenuation grid

$* 2$ Sensitivity of about $30^{\circ}$ in the four polar sectors is reduced down to $1 / 50$ with mechanical attenuation grid

$* 3$ Sensitivity of about $120^{\circ}$ in the 16 polar sectors is reduced down to $1 / 50$ with mechanical attenuation grid

solar wind channel with the mechanical attenuation grid observes solar wind at spin sector groups 1 and 2. Table 10 shows examples of the energy sweep mode allocated to the spin sector group. Numbers from 0 to 5 in the "waveform allocation" correspond to energy sweep modes 0 to 5 in Table 9.

The energy sweep of MIA is as follows: (1) 1 spin (4 s) is equally divided into 64 spin angle sectors; (2) 32 energy steps are swept in each spin angle sector resulting in a sampling time of $4 \mathrm{~s} / 64$ spin sectors/32 energy steps $=\sim 2 \mathrm{~ms}$ ); and (3) 128 energy steps are swept by accumulating 4-spin sets of 32 energy steps/spin. Figure 32 (left) shows an energy sweep waveform of MIA, specifically the voltage applied to the inner sphere for solar wind observation (energy sweep mode 0 or 1 ). In the solar wind, 4 spins are necessary to cover the full 
Table 9 MIA energy sweep

\begin{tabular}{lllll}
\hline Mode & Measurement & Sensitivity control & Spin/cycle & Energy range \\
\hline 0 & Solar wind & OFF & $4(128 \mathrm{steps})$ & $107 \mathrm{eV} / \mathrm{q}-10.3 \mathrm{keV} / \mathrm{q}$ \\
1 & Solar wind & ON & $4(128 \mathrm{steps})$ & $123 \mathrm{eV} / \mathrm{q}-11.6 \mathrm{keV} / \mathrm{q}$ \\
2 & Solar wind & OFF & $2(64 \mathrm{steps})$ & $28.0 \mathrm{eV} / \mathrm{q}-300 \mathrm{eV} / \mathrm{q}(32 \mathrm{steps})$ \\
& & & $2(64 \mathrm{steps})$ & $3.10 \mathrm{keV} / \mathrm{q}-25.8 \mathrm{keV} / \mathrm{q}(32 \mathrm{steps})$ \\
3 & Magnetosphere & OFF & $1(32 \mathrm{steps})$ & $24.0 \mathrm{eV} / \mathrm{q}-25.8 \mathrm{keV} / \mathrm{q}$ \\
4 & Magnetosphere & OFF & $1(32 \mathrm{steps})$ & $21.0 \mathrm{eV} / \mathrm{q}-5.15 \mathrm{keV} / \mathrm{q}$ \\
5 & Magnetosphere & OFF & $1(32 \mathrm{steps})$ & $5.17 \mathrm{keV} / \mathrm{q}-25.8 \mathrm{keV} / \mathrm{q}$
\end{tabular}

Table 10 Examples of energy sweep—sector allocation of MIA

\begin{tabular}{|c|c|c|c|c|c|c|c|c|c|}
\hline \multicolumn{2}{|c|}{ Sector group } & 0 & 1 & 2 & 3 & 4 & & 6 & 7 \\
\hline \multicolumn{2}{|c|}{ Sector } & $0-7$ & $8-15$ & $16-23$ & $24-31$ & $32-39$ & $40-47$ & $48-55$ & $56-63$ \\
\hline No. & Measurement & \multicolumn{8}{|c|}{ Waveform allocation $^{\mathrm{a}}$ (Mode) } \\
\hline 1 & Solar wind & 0 & 1 & 1 & 0 & 0 & 1 & 1 & 0 \\
\hline 2 & Solar wind & 0 & 1 & 1 & 0 & 0 & 2 & 2 & 0 \\
\hline 3 & Magnetosphere & 3 & 3 & 3 & & & 3 & 3 & 3 \\
\hline 4 & Magnetosphere & 4 & 4 & 4 & & 4 & 4 & 4 & 4 \\
\hline 5 & Magnetosphere & 5 & 5 & 5 & 5 & 5 & 5 & 5 & 5 \\
\hline
\end{tabular}

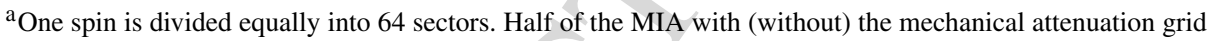
faces the solar wind direction in sectors 8-23 (40-55)

energy range with 128 steps. The full energy range with 32 steps is covered in each spin (energy coverage has some gaps). In this case, 32 steps in 4 different spins are slightly shifted so that 64 energy steps are covered with 2 consecutive spins, and 128 energy steps are covered with 4 consecutive spins. Concerning the attenuation factor, only one pre-defined "top-cap" voltage/inner sphere voltage ratio $\left(V_{t} /\left|V_{i}\right|=1.0\right)$ is used because the G-factor varies too rapidly when the ratio exceeds 1.0 . When we use energy sweep mode 1 , the attenuation is applied to all energy steps to reduce the flux of the solar wind ions. Figure 32 (right) shows the voltage applied to the inner sphere and the "top-cap" for solar wind observation (energy sweep mode 1). Electrical attenuation is enabled by applying the same voltage to the inner sphere and the "top-cap".

According to the MIA mode (modes 1-3; Table 8), the MIA application in the MDP1 continuously computes the VM data and energy spectra $(\mathrm{Et})$ for the mission packets of the L-mode, whereas medium-resolution 3D distribution functions (3D-L2 or SW-L2) are generated for the M-mode. The L-mode mission packets also contain 3D distribution functions (3D-LL), although they are provided in long intervals of (600-3600 s) (Table 11). For the Hmode mission packets, high-resolution 3D distribution functions (SW-L, 3D-L2, or 3D-H) are generated each spin (4 s). The VM consists of the density (n), net flux vector (nV), and pressure tensor $(\mathrm{P})$, which are computed using a lookup table. The detailed format, size and rate of each data product shown in Table 11 are shown in Table 12. For the 3D count data products in the M-mode mission packets, 3D-L2-M1 and 3D-L2-M3, and in the H-mode mission packets, 3D-L2-M1, 89 directions (DIR) are selected from eight spin sectors (SC) 


\section{(a) Spin Sector Definition (FOV direction)} of the ASIC Channels with Mechanical Attenuation Grid 64 spin sectors

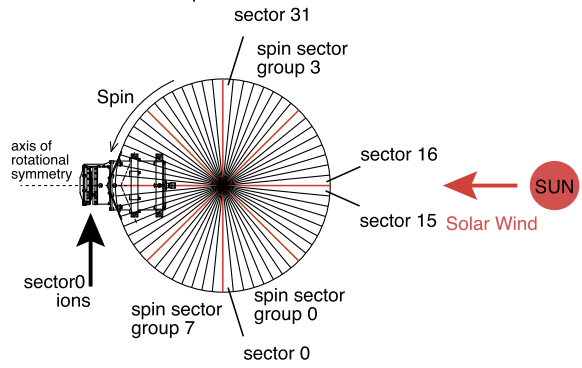

(b) Spin Sector Definition (FOV direction) of the ASIC Channels without Mechanical Attenuation Grid 64 spin sectors

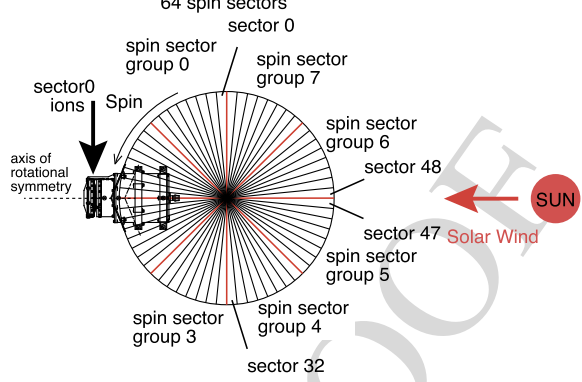

Fig. 31 Definition of the spin sector 0 . Spin Sector 0 is defined when the axis of the rotational symmetry of MIA (dashed line) is pointing away from the Sun. The ion flow direction observed at Spin Sector 0 is indicated by a black arrow and the solar wind ion flow direction is indicated by a red arrow. (a) The solar wind channel with mechanical attenuation grid observes solar wind at spin sector group 1 (from spin sector 8 to 15 ) and spin sector group 2 (from spin sector 16 to spin sector 23). (b) The other channels without the mechanical attenuation grid observe solar wind at spin sector group 5 (from spin sector 40 to 47 ) and spin sector group 6 (from spin sector 48 to spin sector 55)

(a) 64 spin sectors
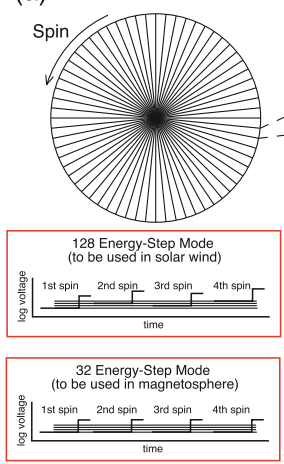

Inner Sphere Voltage

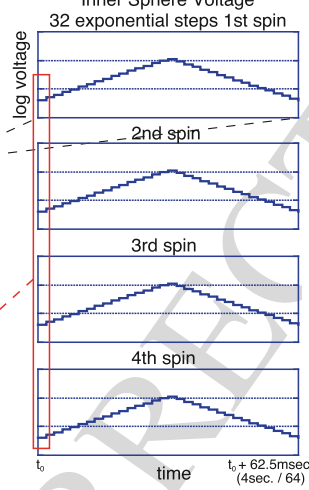

(b)

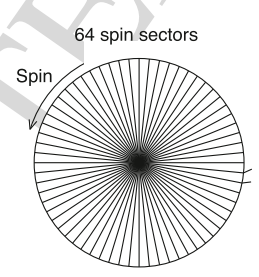

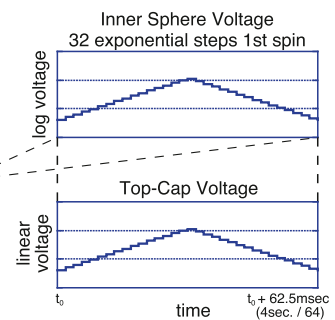

Fig. 32 Energy sweep waveform of MIA. (a) When observing solar wind ions, 128 energy steps are swept by using 4 different 32 energy steps during 4 consecutive spins. (b) When electrical attenuation is enabled, the same voltages are applied to the inner sphere and the top-cap part

$\times 17$ channel $(\mathrm{CH})$ directions. The $\mathrm{VM}$ consists of density $\mathrm{n}$; net flux vectors $\mathrm{nVx}, \mathrm{nVy}$, nVz; and pressure tensors Pxx, Pyy, Pzz, Pxy, Pyz, Pxz. EN in the table represents energy.

\subsubsection{Pre-flight Calibration of MIA}

Pre-flight calibration of the MIA sensor was performed at a calibration facility at the Institute of Space and Astronautical Science/Japan Aerospace Exploration Agency (Wüest et al. 2007). MIA was installed in a vacuum chamber and nitrogen ions were injected. Figure 33 shows schematic diagram of the calibration experiment configuration. The sensor under calibration was installed on a rotation Table 1, which had a rotation axis parallel to the sensor's axis of rotational symmetry. This rotation table was installed on another rotation table ro- 
Table 11 MIA mission data products in the L-mode, M-mode, and H-mode mission packets. Format of each product is shown in Table 12

\begin{tabular}{llll}
\hline MIA operation mode & \\
\hline & MIA Mode 1 & MIA Mode 2 & MIA Mode 3 \\
\hline L-mode & Et-M1 (32s), VM-M1 (4s, 16s) & Et-M2 (4s), VM-M2 (4s) & Et-M3 (4s), VM-M3 (4s) \\
& 3D-LL-M1 (3600s) & 3D-LL-M2 (3600s) & 3D-LL-M3 (600s) \\
M-mode & 3D-L2-M1 (8s) & 3D-L2-M2 (8s) & 3D-L2-M3 (4s) \\
& (MPPE mode =0-5) & 3D-L2-M2 (8s) & (MPPE mode $=0-5)$ \\
M-mode & SW-L2-M1 (4s) & & 3D-L2-M3 (8s) \\
& (MPPE mode =6-8) & (MPPE mode $=6-8)$ \\
H-mode & SW-L-M1 (4s), 3D-L2-M1 (4s) & 3D-H-M2 (4s) & 3D-H-M3 (4s)
\end{tabular}

Table 12 Format, size and rate of the MIA data products. For the 3D count data products, 89 directions (DIR) are selected from 8 spin sectors $(\mathrm{SC}) \times 17$ channel $(\mathrm{CH})$ directions. Velocity moments (VM) consist of density $(\mathrm{n})$, net flux $(\mathrm{nV})(\mathrm{x}, \mathrm{y}, \mathrm{z})$, and pressure $(\mathrm{P})(\mathrm{xx}, \mathrm{yy}, \mathrm{zz}, \mathrm{xy}, \mathrm{yz}, \mathrm{xz})$. EN represents energy

\begin{tabular}{|c|c|c|}
\hline Products & Format (16 bits) & Size/rate \\
\hline Et-M1 (32s) & $\begin{array}{l}8 \text { bits } \times 128(\mathrm{EN}) \\
(\text { Solar wind direction) } \\
8 \text { bits } \times 128(\mathrm{EN}) \times 4 \\
(4 \text {-divided Omni direction) }\end{array}$ & $640 \mathrm{~B} / 160 \mathrm{bps}$ \\
\hline Et-M2, $3(4 \mathrm{~s})$ & $\begin{array}{l}8 \text { bits } \times 16(\mathrm{EN}) \times 4 \\
(4 \text {-divided Omni direction })\end{array}$ & $64 \mathrm{~B} / 128$ bps \\
\hline VM-M1 (4s) & $\begin{array}{l}\text { 16-bit float } \times 10(\mathrm{VM}) \times 2 \\
(\text { Solar wind and Omni })\end{array}$ & $40 \mathrm{~B} / 80 \mathrm{bps}$ \\
\hline VM-M2, 3 (4s) & 16-bit float $\times 10(\mathrm{VM})$ & $20 \mathrm{~B} / 40 \mathrm{bps}$ \\
\hline 3D-LL-M1 (3600s) & 16-bit counter $\times 16 \times 17 \times 32(\mathrm{SC}, \mathrm{CH}, \mathrm{EN})$ & $17 \mathrm{kB} / 39 \mathrm{bps}$ \\
\hline 3D-LL-M2 (3600s) & 16-bit counter $\times 32 \times 17 \times 32(\mathrm{SC}, \mathrm{CH}, \mathrm{EN})$ & $35 \mathrm{kB} / 77 \mathrm{bps}$ \\
\hline 3D-LL-M3 (600s) & 16-bit counter $\times 16 \times 9 \times 32(\mathrm{SC}, \mathrm{CH}, \mathrm{EN})$ & $8 \mathrm{kB} / 110 \mathrm{bps}$ \\
\hline 3D-L2-M1 (8s) & $\begin{array}{l}\text { 16-bit counter } \times 89 \times 32(\mathrm{DIR}, \mathrm{EN}) \\
(\mathrm{Omni})\end{array}$ & $6 \mathrm{kB} / 6 \mathrm{kbps}$ \\
\hline SW-L2-M1 (4s) & $\begin{array}{l}\text { 16-bit counter } \times 4 \times 17 \times 32(\mathrm{SC}, \mathrm{CH}, \mathrm{EN}) \\
(\text { Solar wind })\end{array}$ & $4 \mathrm{kB} / 9 \mathrm{kbps}$ \\
\hline 3D-L2-M2 (8s) & 16-bit counter $\times 16 \times 9 \times 32(\mathrm{SC}, \mathrm{CH}, \mathrm{EN})$ & $9 \mathrm{kB} / 9 \mathrm{kbps}$ \\
\hline 3D-L2-M3 (8s) & 16 -bit counter $\times 89 \times 32(\mathrm{DIR}, \mathrm{EN})$ & $6 \mathrm{kB} / 6 \mathrm{kbps}$ \\
\hline 3D-L2-M3 (4s) & 16 -bit counter $\times 89 \times 16(\mathrm{DIR}, \mathrm{EN})$ & $3 \mathrm{kB} / 6 \mathrm{kbps}$ \\
\hline SW-L-M1 (4s) & 16 -bit counter $\times 16 \times 17 \times 32(\mathrm{SC}, \mathrm{CH}, \mathrm{EN})$ & $17 \mathrm{kB} / 35 \mathrm{kbps}$ \\
\hline 3D-L2-M1 (4s) & 16 -bit counter $\times 89 \times 32(\mathrm{DIR}, \mathrm{EN})$ & $6 \mathrm{kB} / 11 \mathrm{kbps}$ \\
\hline $3 \mathrm{D}-\mathrm{H}-\mathrm{M} 2(4 \mathrm{~s})$ & 16 -bit counter $\times 32 \times 17 \times 32(\mathrm{SC}, \mathrm{CH}, \mathrm{EN})$ & $35 \mathrm{kB} / 70 \mathrm{kbps}$ \\
\hline 3D-H-M3 (4s) & 16-bit counter $\times 16 \times 17 \times 32(\mathrm{SC}, \mathrm{CH}, \mathrm{EN})$ & $17 \mathrm{kB} / 34 \mathrm{kbps}$ \\
\hline
\end{tabular}

tation Table 2 in which the rotation axis was perpendicular to both the beam line and the rotation axis of rotation Table 1 . Most of the data were obtained using $6 \mathrm{keV}$ nitrogen ion beams because the beam profile was uniform and stable. 
Table 13 Summary of MIA performance

\begin{tabular}{|c|c|}
\hline \multirow[t]{4}{*}{ Field of view } & $3.8^{\circ} \times 90^{\circ}$ (high G-factor, solar wind) \\
\hline & $5.1^{\circ} \times 90^{\circ}$ (low G-factor, solar wind) \\
\hline & $9.6^{\circ} \times 270^{\circ}$ (high G-factor, Mercury ion) \\
\hline & $6.4^{\circ} \times 270^{\circ}$ (low G-factor, Mercury ion) \\
\hline \multirow[t]{2}{*}{ Angular resolution } & $5.625^{\circ} \times 5.625^{\circ}($ solar wind $)$ \\
\hline & $22.5^{\circ} \times 22.5^{\circ}$ (Mercury ion) \\
\hline Energy range & $15 \mathrm{eV} / \mathrm{q}-29 \mathrm{keV} / \mathrm{q}$ \\
\hline \multirow[t]{4}{*}{ Energy resolution } & $\Delta E / E \sim 8.3 \%$ (FWHM, high G-factor, sol \\
\hline & $\Delta E / E \sim 2.2 \%$ (FWHM, low G-factor, sola \\
\hline & $\Delta E / E \sim 12.7 \%$ (FWHM, high G-factor, \\
\hline & $\Delta E / E \sim 3.6 \%$ (FWHM, low G-factor, Me \\
\hline \multirow[t]{2}{*}{ Time resolution } & (32 energy steps) 4 s/3D distribution funct \\
\hline & (128 energy steps) $16 \mathrm{~s} / 3 \mathrm{D}$ distribution \\
\hline \multicolumn{2}{|l|}{ Geometrical factor } \\
\hline High G-factor mode & $3.39 \times 10^{-6} \mathrm{~cm}^{2} \mathrm{sreV} / \mathrm{eV}$ (solar wind) \\
\hline$\left(5.625^{\circ}: \mathrm{SW} 22.5^{\circ}: \mathrm{MI}\right)$ & $4.64 \times 10^{-4} \mathrm{~cm}^{2} \mathrm{sreV} / \mathrm{eV}$ (Mercury ion) \\
\hline Low G-factor mode & $2.81 \times 10^{-7} \mathrm{~cm}^{2} \mathrm{sreV} / \mathrm{eV}$ (solar wind) \\
\hline$\left(5.625^{\circ}: \mathrm{SW} 22.5^{\circ}: \mathrm{MI}\right)$ & $1.23 \times 10^{-5} \mathrm{~cm}^{2} \mathrm{sreV} / \mathrm{eV}$ (Mercury ion) \\
\hline \multicolumn{2}{|l|}{ Mass } \\
\hline Power & $2.96 \mathrm{~W}$ \\
\hline Dimensions & $180 \mathrm{~mm} \times 254 \mathrm{~mm} \times 146 \mathrm{~mm}$ \\
\hline \multirow[t]{3}{*}{ Data rate } & 0.11 kbits/s (L-mode) \\
\hline & $2.5 \mathrm{kbits} / \mathrm{s}$ (M-mode) \\
\hline & 17 kbits/s (H-mode) \\
\hline
\end{tabular}

Table 13 gives a summary of MIA performance data determined by pre-flight calibration. The definition for the quantities in this table is general and is the same as that used by Wüest et al. (2007). Figure 34(a) and (b) compares the E- $\alpha$ characteristics of MIA with and without electrical attenuation. When electrical attenuation is enabled, both the angular spread and energy spread become narrower and the measured ion energy increases. Since angular resolution and energy resolution become higher, electrical attenuation is appropriate for solar wind ion observation. The G-factor is reduced to about 1/50 where the mechanical attenuation grid is installed. Figure 34(b) and (c) compares the E- $\alpha$ characteristics of MIA with and without a mechanical attenuation grid. It is clear that part of the $\mathrm{E}-\alpha$ contour with large $\alpha$ angle is reduced by the mechanical attenuation grid. Because the attenuation grid is placed only at the bottom part of the entrance aperture and the upper part is closed as shown in Fig. 29, the ions entering MIA with large $\alpha$ angles are blocked. Consequently, the center of the measured $\alpha$ angle becomes smaller and that of the measured energy becomes lower compared to those without a mechanical attenuation grid. 
Fig. 33 Schematic diagram showing the configuration of MIA calibration. Rotation Table 1 is installed on Rotation Table 2. The ion entrance angles $\alpha$ and $\theta$ with the definitions of their positive and negative directions are shown

(a)

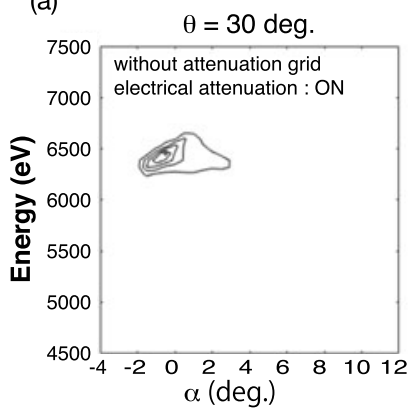

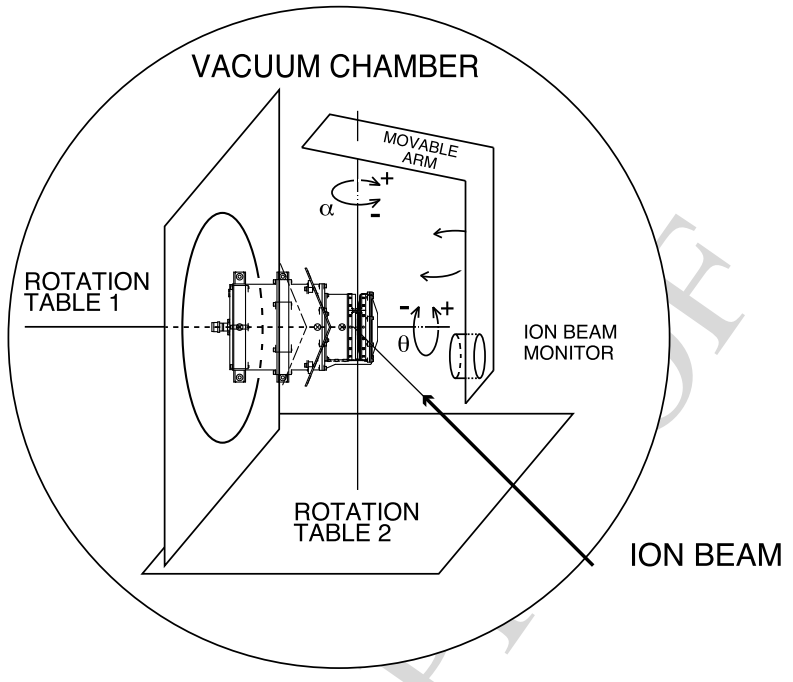

(b)

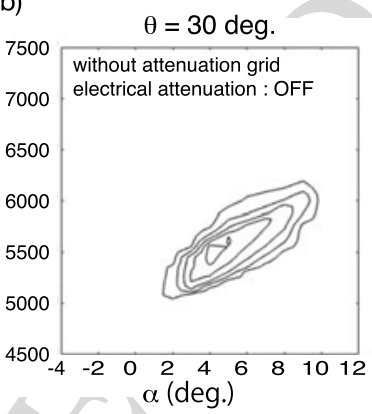

(c)

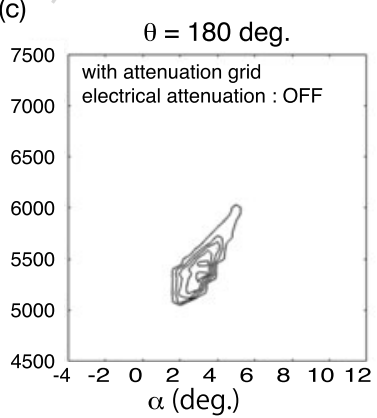

Fig. 34 Examples of E- $\alpha$ contour. An ion beam with a sufficiently large area was injected at $\theta=30^{\circ}$ for panels (a) and (b) and $\theta=180^{\circ}$ for panel (c). The counts are normalized by the maximum count in each E- $\alpha$ contour; five contour lines with levels of $0.1,0.3,0.5,0.7$ and 0.9 are shown. (a) Without the mechanical attenuation grid; the electrical attenuation is ON. (b) Without the mechanical attenuation grid; the electrical attenuation is OFF. (c) With the mechanical attenuation grid; the electrical attenuation is OFF

Figure 35(a) shows the $\theta$ (azimuthal angle) resolution of MIA. The $\theta$ angle coverage of all anode channels are shown when electrical attenuation is OFF (left panel) and ON (right panel). It is clear that the sensitivity is reduced where the mechanical attenuation grid is installed at the entrance of the analyzer (CH38-CH57). Figure 35(b) and (c) shows the $\theta$ angle coverage in greater detail. When the electrical attenuation is ON (panel (c)), the $\theta$ angular resolution becomes much higher than that when the electrical attenuation OFF (panel (b)).

Figure 36 shows the G-factor of MIA for all 64 channels. MIA has physical supports across the entrance aperture every $120^{\circ}$. The light and thick blue boxes indicate the channels affected by these physical supports (CH15, CH16, CH36, CH37, CH58 and CH59). The G-factor is reduced to about 1/50 where the mechanical attenuation grid is installed (CH38-CH57). Although the geometrical attenuation factor of the mechanical grid is $1 / 10$ (Fig. 29(c)), the G-factor reduction is $\sim 1 / 50$ because the mechanical grid also changes the angular and energy characteristics of MIA as shown in Fig. 34. Slight variation of the 
(a)

Fig. $35 \theta$ (azimuthal angle) resolution of MIA. (a) The $\theta$ angle coverage of each anode channel is shown (left panel: electrical attenuation OFF; right panel: electrical attenuation $\mathrm{ON}$ ). (b) The $\theta$ angle coverage of anode channels $\mathrm{CH} 23-\mathrm{CH} 34$ without the mechanical attenuation grid and the electrical attenuation is OFF. (c) The $\theta$ angle coverage of anode channels CH42-CH53 with the mechanical attenuation grid and the electrical attenuation is $\mathrm{ON}$

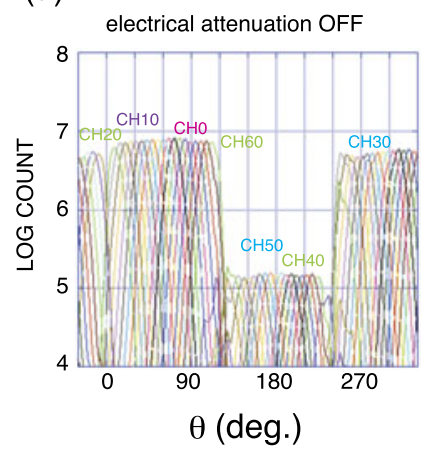

(b)

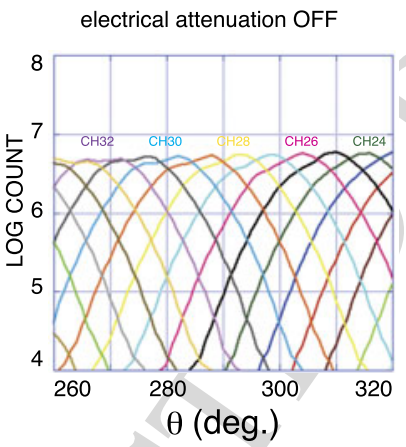

electrical attenuation $\mathrm{ON}$

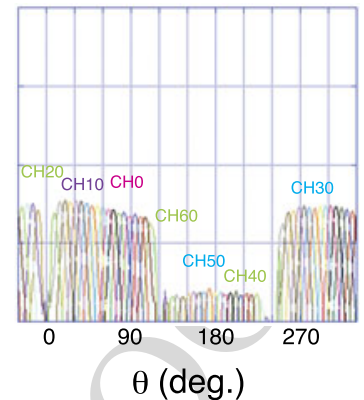

(c)

electrical attenuation $\mathrm{ON}$

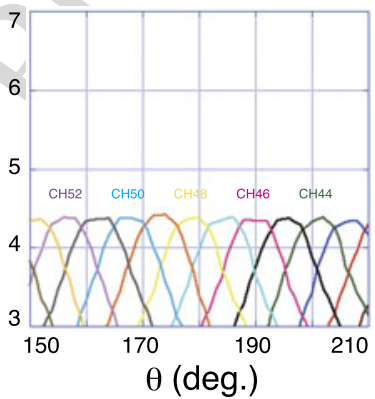

Fig. 36 Geometrical factor of MIA. Red dots show G-factor without electrical attenuation. Blue dots show Gfactor with electrical attenuation (energy sweep mode 1). The light and thick blue boxes indicate the channels affected by the physical supports across the entrance aperture. Channel 15 is connected to an annular anode where no ions from the analyzer are expected to enter for monitoring the background count (Saito et al. 2017). Channel 16, which is not connected to an anode is used to monitor the electrical background noise. The relationship between the ion entrance angle $\theta$ (Fig. 33) and the corresponding ASIC channel is also shown

\section{르 Springer}


G-factor also exists among the different channels without the mechanical attenuation grid with large values around $\mathrm{CH} 0$ and small values around $\mathrm{CH} 31$. The variation of the analyzer constant was also occurred simultaneously with the variation of the G-factor. This variation can be explained by the slight inclination of the inner sphere with respect to the outer sphere where the inclination angle was as small as $0.2^{\circ}$ that is within the manufacturing tolerance.

\subsubsection{Near-Earth Commissioning Results of MIA}

The low voltage part of MIA was turned on for the first time on November 25, 2018, about one month after the launch. No problems were identified during the low voltage function tests, which included calibration pulse injection into all channels of the pre-amplifier.

The high voltage tests were performed on July 3 and 4, 2019, about eight months after the launch when Mio was in the solar wind. High voltage up to $+2500 \mathrm{~V},-3610 \mathrm{~V}$, and $-2471 \mathrm{~V}$ were successfully tested for stepping high voltage power supply connected to the "top-cap" (SVG), stepping high voltage power supply connected to the inner sphere (SVS), and high voltage power supply connected to the MCP detector (MHV), respectively. Dark counts of the MCP were observed indicating that the detector part of MIA was functioning normally. Because Mio is surrounded by the MMO Sunshield and Interface Structure (MOSIF) during the cruise phase, most parts of the MIA's FOV is blocked by MOSIF. Although all of the high voltage necessary for observation were successfully applied to MIA, no solar wind ion signature was observed because the solar wind ion thermal velocity was much lower than its bulk velocity, and MOSIF blocked the solar wind ions to enter MIA.

Because MIA is able to measure hot plasmas in the magnetospheres of Earth and Mercury and near-Venus when the plasma thermal velocity is higher than its bulk velocity, MIA will be turned on during the Earth fly-by and the Venus/Mercury fly-bys before arriving at Mercury in December 2025.

\subsection{MSA}

\subsubsection{Instrument Description of MSA}

MSA, part of the MPPE particle consortium, is dedicated to plasma composition measurement onboard Mio (Delcourt et al. 2016). The main objectives of this instrument are (1) to study the role and efficiency of the solar wind and planetary surface as sources of plasma for the Hermean magnetosphere; (2) to study the transport, acceleration and loss of plasma in the Hermean magnetosphere, particularly for investigating the dynamics of heavy ions of planetary origin that have large gyroradii and large gyroperiods as compared to the characteristic scales of the magnetosphere; (3) to contribute to the understanding of magnetosphere electrodynamics, substorms, and the nature of current carriers; (4) to analyze the interaction of magnetospheric plasma with the planetary surface and to study the processes by which particles escape from the surface and access the magnetosphere; (5) to provide data that will help to identify Mercury's surface composition; (6) to monitor the solar wind and to study interstellar pick-up ions. To achieve these issues, MSA will provide 3D distribution functions in one Mio spin (4 s). In addition, in contrast to Earth where ions of planetary origin are essentially $\mathrm{O}^{+}$and $\mathrm{He}^{+}$, a wide variety of species populate Mercury's magnetosphere owing to various interaction processes with the planet surface (such as solar wind sputtering, micro-meteoritic bombardment, and thermal desorption). To characterize these populations, a spectrometer with enhanced mass resolution capability is necessary; hence, the "reflectron" design was adopted for MSA. 
Fig. 37 MSA flight model delivered to Mio system in June 2014

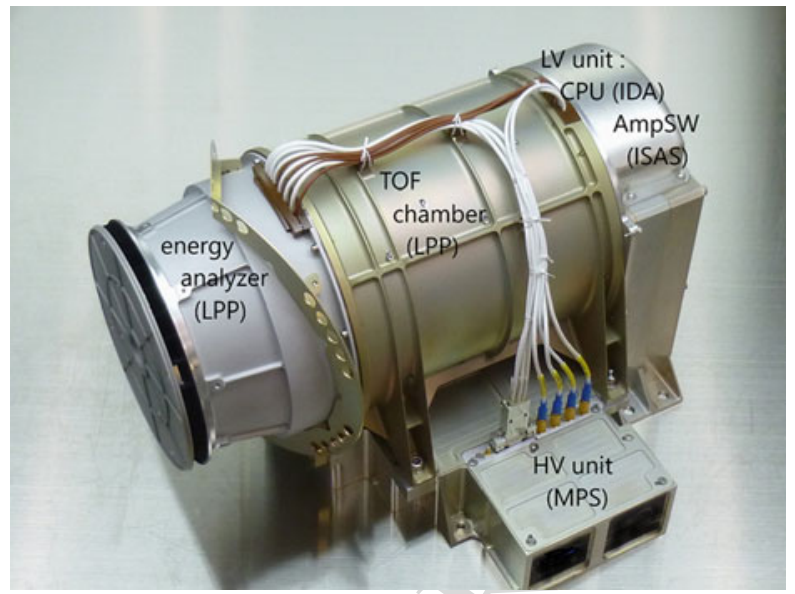

Fig. 38 Block diagram of MSA

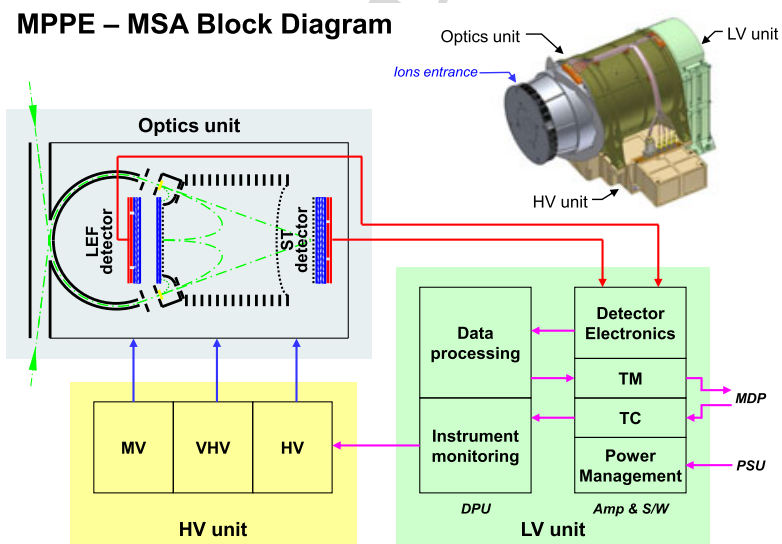

As summarized in Fig. 37, MSA is the result of collaboration of four different teams: (1) ISAS (Sagamihara, Japan; PI), which provided the amplifier board and spacewire interface; (2) LPP (Palaiseau, France; Co-PI), which provided the electrostatic optics; (3) MPS (Göttingen, Germany), which provided the high voltage power supplies; and (4) IDA (Braunschweig, Germany), which provided the dedicated central processing unit (CPU) board. A block diagram of MSA is shown in Fig. 38. MSA has dimensions of $325 \mathrm{~mm} \times 287 \mathrm{~mm} \times 232 \mathrm{~mm}$, a weight of $4.46 \mathrm{~kg}$, and medium telemetry mode power of $9.1 \mathrm{~W}$.

This instrument, which is derived from CAPS-IMS onboard CASSINI (Young et al. 2004), consists of a 2D mass spectrometer of cylindrical symmetry with respect to the main axis as illustrated in Fig. 39. It operates over a large energy range of $\sim 1 \mathrm{eV} / \mathrm{q}$ to $\sim 38 \mathrm{keV} / \mathrm{q}$ with an instantaneous FOV of $5^{\circ} \times 260^{\circ}$. The MSA entrance (blackened for UV rejection) features 32 angular sectors of $11.25^{\circ}, 9$ of which are blinded by the Mio magnetometer mast. After MSA entrance, a spherical analyzer enables measurement of the full range of ion energies in 1/32 of Mio spin. The grounded external electrode of this analyzer is designed in three mechanical parts, including polarization of the central one leading to de-focusing (thus, effective control) of the incoming ion flux. After the energy analysis, ions are ac- 
Fig. 39 Schematic illustration of MSA principles

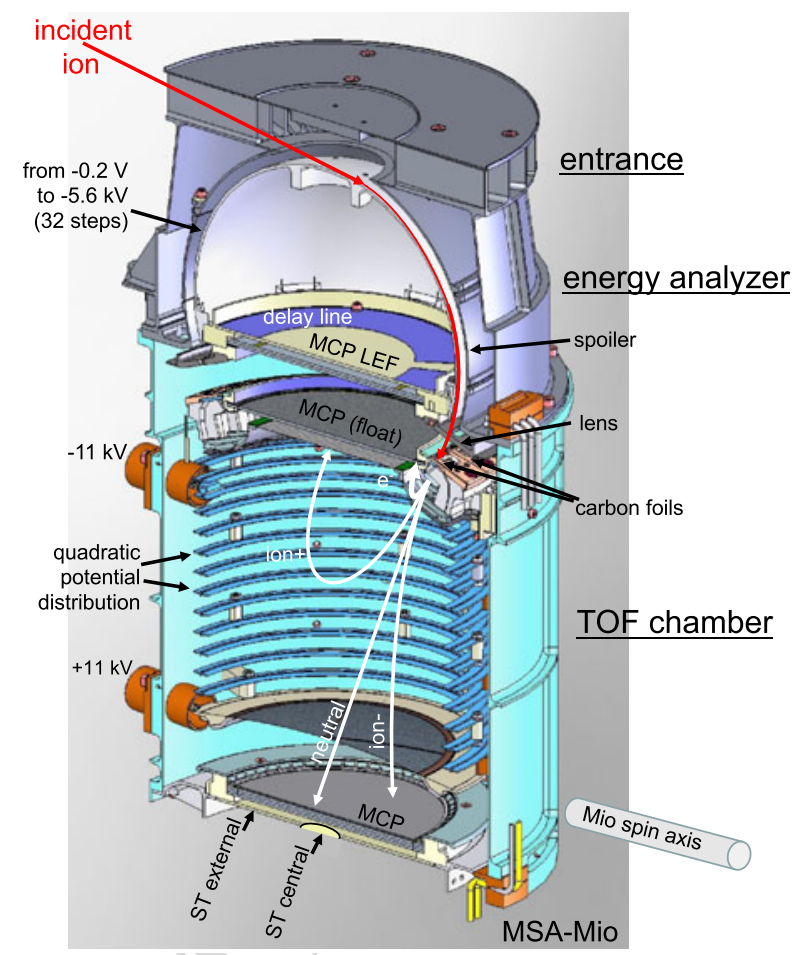

celerated $( \pm 12 \mathrm{kV}$ in maximum operation voltage) toward a time of flight (TOF) chamber polarized with a linear electric field (LEF) along the instrument's main axis. This leads to isochronous (independent of energy) TOF for reflected ions up to $\pm 12 \mathrm{keV}$ for maximum operation voltage (Delcourt et al. 2016), hence, enhanced mass resolution $(m / \Delta m>40)$ over a large range of masses up to $60 \mathrm{amu}$. The straight-through (ST) particles are collected at the bottom end of the MSA TOF chamber, and a lower mass resolution is obtained that is somewhat improved using the central detector with reduced path lengths rather than the external one (Fig. 39). Details of the TOF signal of MSA has been described by Saito et al. (2017).

Figure 40 shows the thermal shield of MSA. Some selected parts of the MSA electrostatic optics are shown in Figs. 41 and 42. In particular, Fig. 42 shows the 21 angular sectors of entry equipped with carbon foils at the TOF chamber entrance. This figure also shows the bi-metallized Hamamatsu MCP at the back of the energy analyser, which enables differentiation of the MCP gain on the outer edge, where START electrons are collected, from that in the inner part, where LEF counts are obtained.

The MSA high voltage power Supply (HVPS Figs. 43 and 44) has 10 different sub-units supplying voltages of $30 \mathrm{~V}$ to $\pm 12 \mathrm{kV}$. These voltages can be individually modified by commands during flight. A radiation hard Actel RTSX FPGA controls the output-voltage of each sub-unit, whereas monitoring and commanding of the voltages are performed by the MSA CPU. The sub-units comprise the power supplies for (i) the 2 MCP stacks of MSA, (ii) the adjustable MCP anode-voltages, (iii) the two flux spoilers, (iv) MSA reflectron with an operational voltage range up to $\pm 12 \mathrm{kV}$, and (v) a stepping voltage for the energy analyzer with a dynamic range between $0.1 \mathrm{~V}$ and $5.6 \mathrm{kV}$ and a settling time $<1 \mathrm{~ms}$. 
Fig. 40 Thermal shield of MSA
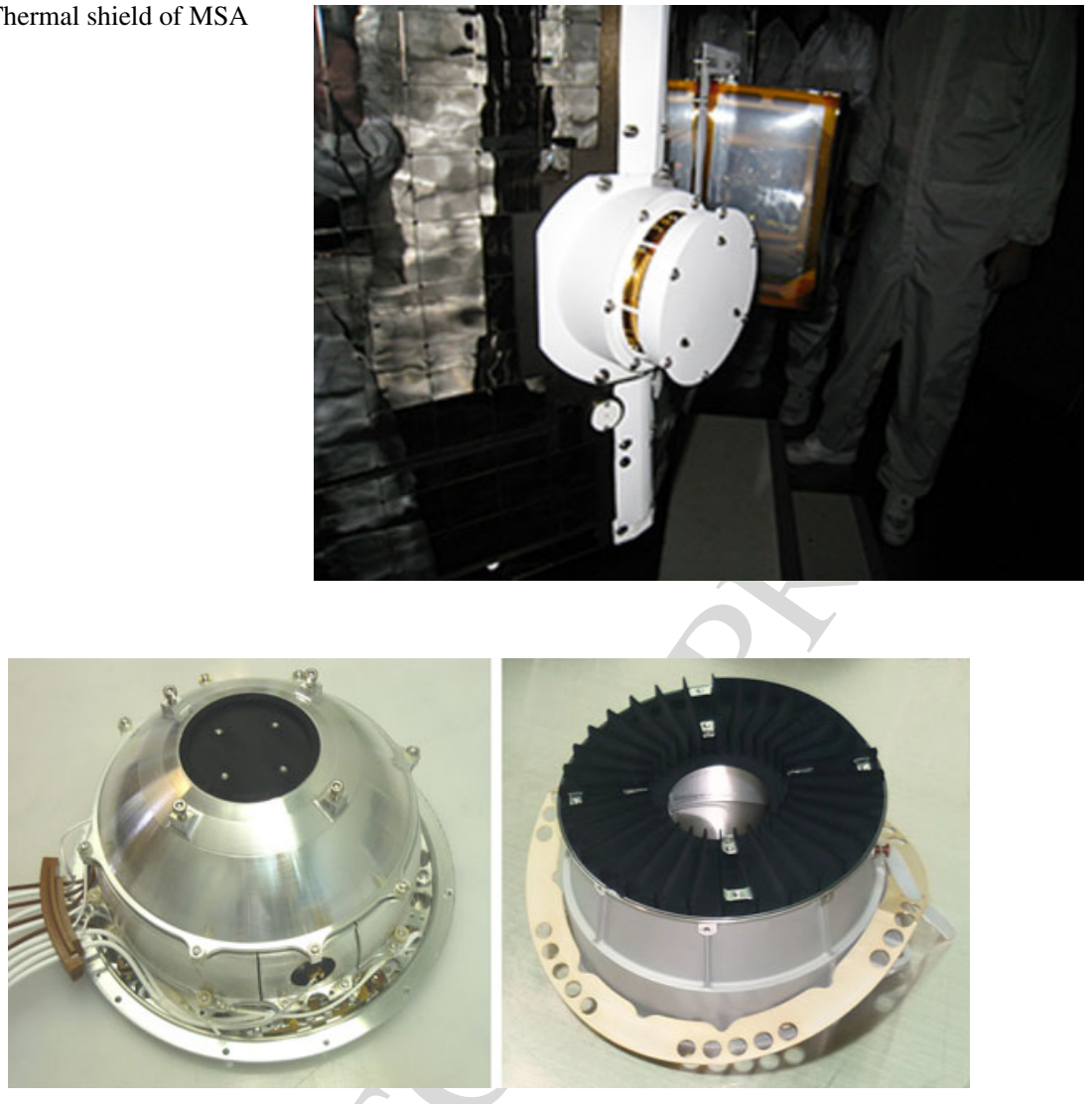

Fig. 41 Copper sulfide blackened energy analyzer (left) and entrance section (right)

Fig. 42 Energy analyzer (left, viewed from the back), and TOF chamber (right, view from top)
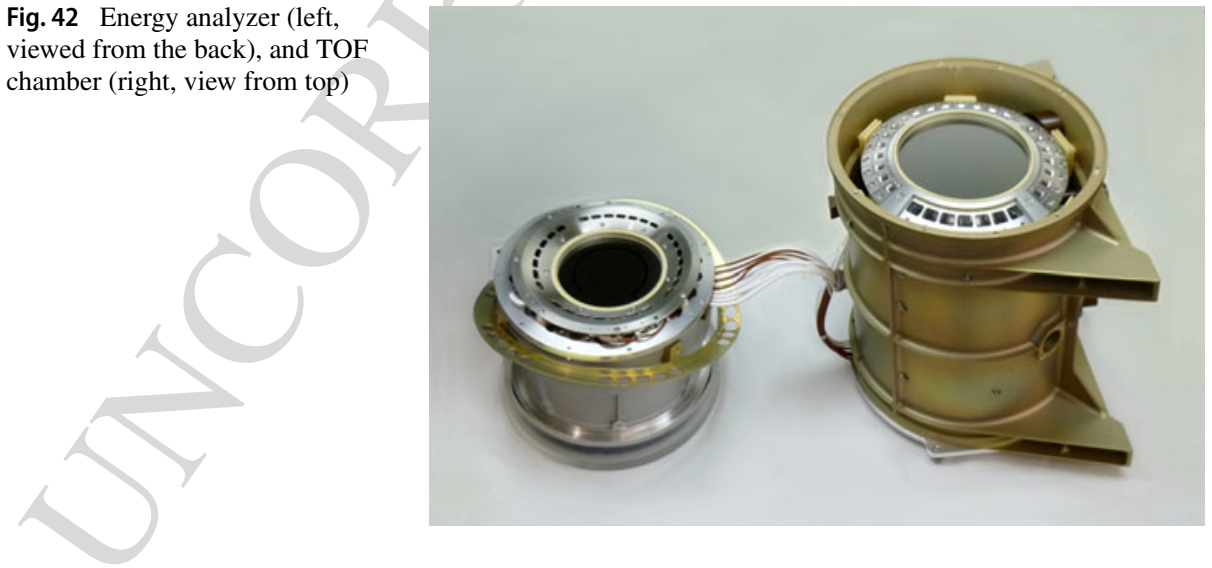

The CPU (Figs. 45 and 46) performs instrument control and dedicated data processing. The advanced design is based on the AT697, a $48 \mathrm{MHz}$ LEON2-FT fault tolerant SPARC processor architecture implemented in radiation hard ASIC, including Triple Mod-

\section{Springer}


2001

2002

2003

2004

2005

2006

2007

2008

2009

2010

2011

2012

2013

2014

2015

2016

2017

2018

2019

2020

2021

2022

2023

2024

2025

2026

2027

2028

2029

2030

2031

2032

2033

2034

2035

2036

2037

2038

2039

2040

2041

2042

2043

2044

2045

2046

2047

2048

2049

2050
Fig. 43 MSA HPVS (top view)

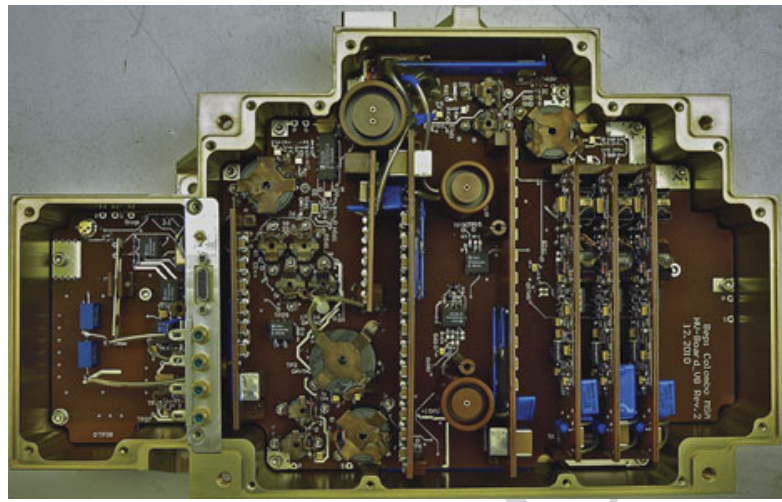

Fig. 44 MSA HVPS (bottom view)

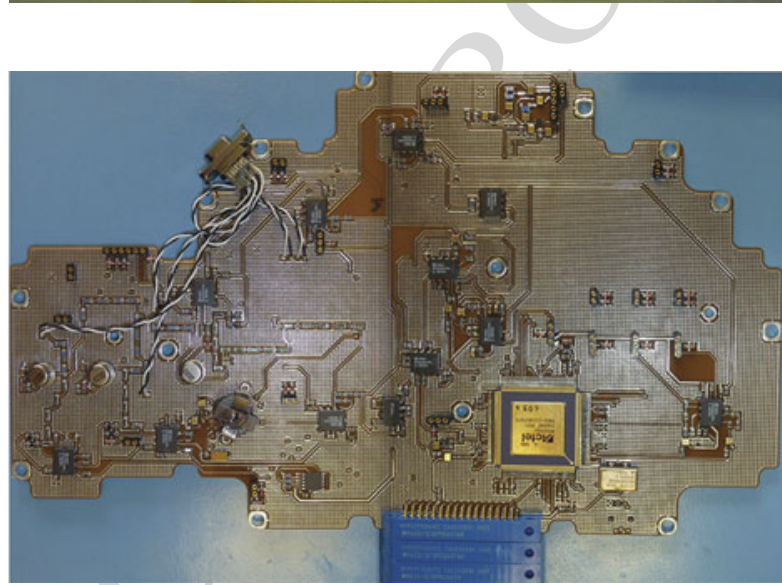

Fig. 45 MSA CPU (top view)

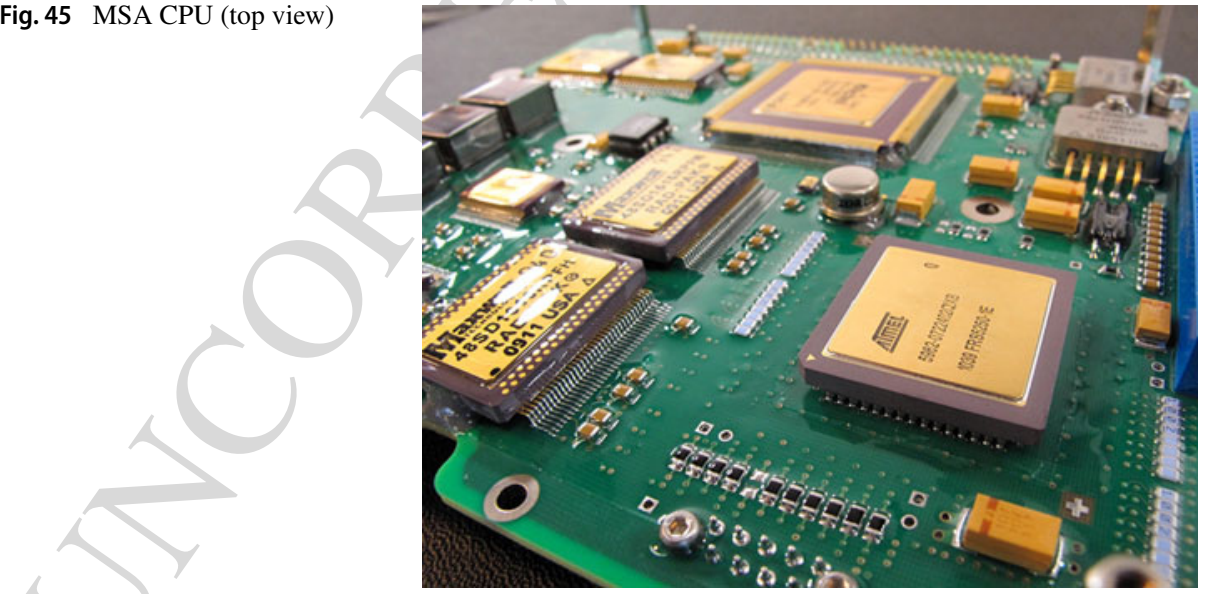

ular Redundancy (TMR) and advanced Error Detection And Correction (EDAC) against SEU errors. Together with a radiation hardened, one-time programmable FPGA (Microsemi RTAX), the processor is employed for high-level instrument control and monitoring, control of sensor electronics and compression of scientific data. All internal interfaces and glue 
Fig. 46 MSA CPU (bottom view)
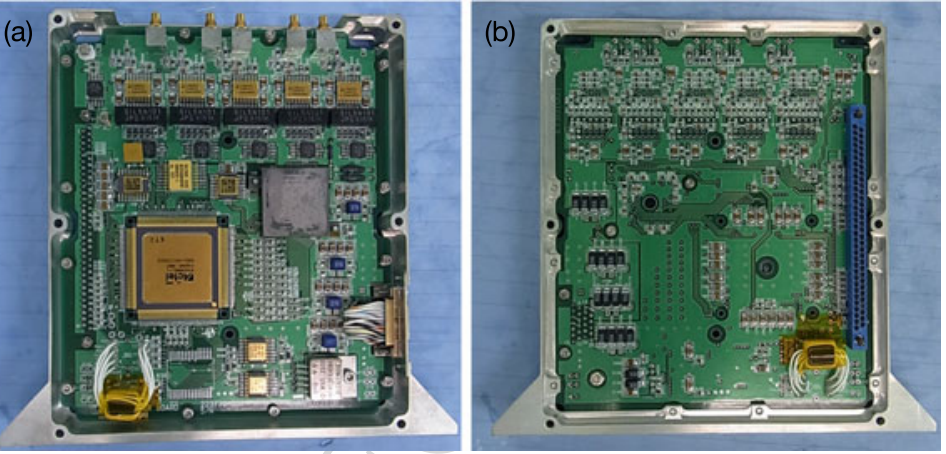

Fig. 47 MSA TOF signal amplifiers and SpaceWire interface. (a) top view (b) bottom view

logic are embedded in the radiation hardened RTAX system FPGA to achieve the highest reliability. Program code images can be uploaded to the non-volatile memory and executed in the EDAC protected SDRAM module.

Figure 47 shows the top view (panel (a)) and bottom view (panel (b)) of the amplifier board and spacewire interface. Five charge signals from the MCP anodes are fed into five fast transistor amplifiers and the amplified signals are discriminated by constant fraction discriminators. A custom gate array with a titanium radiation shield calculates and buffers the signal timing with a time resolution of 781.25 ps. An FPGA Actel RTAX2000 that communicates with the CPU interfaces with MDP1, controls the custom gate array, and processes the signal timing calculated by the custom gate array.

The performance of the MSA instrument are given in Table 14. Its capability is summarized as follows. (1) ST data: high temporal resolution, high sensitivity measurements and medium mass resolution $(m / \Delta m$ up to $\sim 10)$ to focus on major ion species and allow rapid analysis of magnetospheric phenomena, which makes MSA appropriate for plasma analysis. (2) LEF data: high mass resolution measurements with $m / \Delta m$ up to 60 for ions with energies smaller than the MSA operation voltage, up to a maximum of $12 \mathrm{kV}$ because ions with larger energies cannot be turned around by the LEF and are detected on the ST MCP. This 


\section{Table 14 Summary of MSA} performance

\begin{tabular}{|c|c|}
\hline Field of view & $5^{\circ} \times 260^{\circ}$ \\
\hline Angular resolution & $5^{\circ} \times 11.25^{\circ}$ \\
\hline Energy range & $1 \mathrm{eV} / \mathrm{q}-38 \mathrm{keV} / \mathrm{q}$ \\
\hline Energy resolution & $\Delta E / E=8 \%$ \\
\hline $\mathrm{k}$-factor & 6.85 \\
\hline Mass range & $1-60 \mathrm{amu}$ \\
\hline \multirow[t]{2}{*}{ Mass resolution } & $m / \Delta m>40(<13 \mathrm{keV} / \mathrm{q})$ \\
\hline & $m / \Delta m=10(>13 \mathrm{keV} / \mathrm{q})$ \\
\hline \multirow[t]{4}{*}{ Time resolution } & 3D distribution function in $4 \mathrm{~s}$ \\
\hline & (32 energy steps) \\
\hline & 3D distribution function in $8 \mathrm{~s}$ \\
\hline & (64 energy steps) \\
\hline \multirow[t]{2}{*}{ Geometrical factor (21 windows) } & $7 \times 10^{-3} \mathrm{~cm}^{2} \mathrm{sreV} / \mathrm{eV}(\mathrm{ST})$ \\
\hline & $5 \times 10^{-4} \mathrm{~cm}^{2} \mathrm{sreV} / \mathrm{eV}(\mathrm{LEF})$ \\
\hline \multicolumn{2}{|l|}{ Mass } \\
\hline Power & $9.1 \mathrm{~W}$ \\
\hline Dimensions & $325 \mathrm{~mm} \times 287 \mathrm{~mm} \times 232 \mathrm{~mm}$ \\
\hline \multirow[t]{3}{*}{ Data rate } & $0.15 \mathrm{kbits} / \mathrm{s}$ (L-mode) \\
\hline & 1.4 kbits/s (M-mode) \\
\hline & 25 kbits/s (H-mode) \\
\hline
\end{tabular}

process focuses on ions of planetary origin and enables precise composition measurement, which demonstrates the appropriateness of MSA for planetology studies.

\subsubsection{Operation Mode and Data Products of MSA}

MSA energy sampling features 32 energy steps over the full energy range $(1 \mathrm{eV} / \mathrm{q}$ to $38 \mathrm{keV} / \mathrm{q}$ ) of the instrument during one spin sector: $1 / 32$ spin $=125 \mathrm{~ms}$. To more effectively cover the wide energy range of MSA, two distinct sets of interleaved energy steps are used during two consecutive spins. At the exit of the energy analyzer, ions enter the TOF chamber, and secondary electrons collected as START pulses are emitted upon crossing the carbon foils. Upon impact of the ions on either the LEF or ST detectors, STOP pulses are recorded and the resulting coincidences (i.e., the given STOP signal associated with the given START signal) provide information on the particle TOF or, equivalently, on their mass to charge $(\mathrm{m} / \mathrm{q})$ ratio.

Owing to the differences in ST and LEF spectra, two different approaches are implemented. For LEF particles, TOF spectra with $2 \times 32$ energies (corresponding to the two distinct sets of interleaved steps described above) are produced every 16 spins (64 s). For ST particles, once the STOP-START calculations are completed, allocation of the ion mass is performed onboard using fixed mass groups. In total, 15 count rate matrices are built. The first four are "all ions" or "all START counts", $\mathrm{H}^{+}, \mathrm{He}^{2+}$, and heavy ions including all ions with $\mathrm{m} / \mathrm{q}$ ratios larger than 2 . These matrices are produced at each $4 \mathrm{~s}$ interval and only these first four count rate matrices are transmitted in the Mio low telemetry mode. The next 11 count rate matrices relate to $\mathrm{He}^{+}, \mathrm{C}^{-}, \mathrm{O}^{-}, \mathrm{C}, \mathrm{N}, \mathrm{O}, \mathrm{Na}, \mathrm{Si}, \mathrm{S}, \mathrm{K}$, and $\mathrm{Fe}$ ions and are produced at each 16 spins (64 s). Please note that negative ions here are not external ions (the voltage configuration of MSA energy analyzer does not admit negative ions) but ions 
Table 15 MSA normal operation mode. M\#: count rate matrix of given ion species; A: anode (or entrance window); S: spin sector (32 in one spin); E: energy step (32 in one spin sector); V: velocity direction (36 view directions); T: time of flight

\begin{tabular}{|c|c|c|c|c|c|c|c|}
\hline \multirow[t]{2}{*}{ Name } & \multirow[t]{2}{*}{ Content } & \multicolumn{2}{|l|}{ Internal (32 bit) } & \multicolumn{2}{|c|}{ External (16 bit) } & \multicolumn{2}{|c|}{ Time resolution } \\
\hline & & Dimension & kbit & Dimension & kbit & Int & Med \\
\hline M0 & Starts & $32 \mathrm{~S} \times 32 \mathrm{E} \times 21 \mathrm{~A}$ & 688 & $36 \mathrm{~V} \times 64 \mathrm{E}$ & 37 & $4 \mathrm{~s}$ & $24 \mathrm{~s}$ \\
\hline A0 & Starts & & & $21 \mathrm{~A} \times 32 \mathrm{~S}$ & 11 & $64 s$ & $256 \mathrm{~s}$ \\
\hline M1 & Protons & $32 \mathrm{~S} \times 32 \mathrm{E} \times 21 \mathrm{~A}$ & 688 & $36 \mathrm{~V} \times 64 \mathrm{E}$ & 37 & $4 \mathrm{~s}$ & $24 \mathrm{~s}$ \\
\hline M2 & $\mathrm{He}^{++}$ & $32 \mathrm{~S} \times 32 \mathrm{E} \times 21 \mathrm{~A}$ & 688 & $36 \mathrm{~V} \times 64 \mathrm{E}$ & 37 & $4 \mathrm{~s}$ & $48 \mathrm{~s}$ \\
\hline M3 & HeavyIons & $32 \mathrm{~S} \times 32 \mathrm{E} \times 21 \mathrm{~A}$ & 688 & $36 \mathrm{~V} \times 64 \mathrm{E}$ & 37 & $4 s$ & $48 \mathrm{~s}$ \\
\hline M4 & OtherIons & $32 \mathrm{~S} \times 64 \mathrm{E} \times 21 \mathrm{~A}$ & 1376 & $36 \mathrm{~V} \times 64 \mathrm{E}$ & 37 & $64 \mathrm{~s}$ & $256 \mathrm{~s}$ \\
\hline M5 & OtherIons & $32 \mathrm{~S} \times 64 \mathrm{E} \times 21 \mathrm{~A}$ & 1376 & $36 \mathrm{~V} \times 64 \mathrm{E}$ & 37 & $64 \mathrm{~s}$ & $256 \mathrm{~s}$ \\
\hline M6 & OtherIons & $32 \mathrm{~S} \times 64 \mathrm{E} \times 21 \mathrm{~A}$ & 1376 & $36 \mathrm{~V} \times 64 \mathrm{E}$ & 37 & $64 \mathrm{~s}$ & $256 \mathrm{~s}$ \\
\hline M7 & OtherIons & $32 \mathrm{~S} \times 64 \mathrm{E} \times 21 \mathrm{~A}$ & 1376 & $36 \mathrm{~V} \times 64 \mathrm{E}$ & 37 & $64 \mathrm{~s}$ & $256 \mathrm{~s}$ \\
\hline M8 & OtherIons & $32 \mathrm{~S} \times 64 \mathrm{E} \times 21 \mathrm{~A}$ & 1376 & $36 \mathrm{~V} \times 64 \mathrm{E}$ & 37 & $64 \mathrm{~s}$ & $256 \mathrm{~s}$ \\
\hline M9 & OtherIons & $32 \mathrm{~S} \times 64 \mathrm{E} \times 21 \mathrm{~A}$ & 1376 & $36 \mathrm{~V} \times 64 \mathrm{E}$ & 37 & $64 \mathrm{~s}$ & $256 \mathrm{~s}$ \\
\hline M10 & OtherIons & $32 \mathrm{~S} \times 64 \mathrm{E} \times 21 \mathrm{~A}$ & 1376 & $36 \mathrm{~V} \times 64 \mathrm{E}$ & 37 & $64 \mathrm{~s}$ & $256 \mathrm{~s}$ \\
\hline M11 & OtherIons & $32 \mathrm{~S} \times 64 \mathrm{E} \times 21 \mathrm{~A}$ & 1376 & $36 \mathrm{~V} \times 64 \mathrm{E}$ & 37 & $64 \mathrm{~s}$ & $256 \mathrm{~s}$ \\
\hline M12 & OtherIons & $32 \mathrm{~S} \times 64 \mathrm{E} \times 21 \mathrm{~A}$ & 1376 & $36 \mathrm{~V} \times 64 \mathrm{E}$ & 37 & $64 \mathrm{~s}$ & $256 \mathrm{~s}$ \\
\hline M13 & OtherIons & $32 \mathrm{~S} \times 64 \mathrm{E} \times 21 \mathrm{~A}$ & 1376 & $36 \mathrm{~V} \times 64 \mathrm{E}$ & 37 & $64 \mathrm{~s}$ & $256 \mathrm{~s}$ \\
\hline M14 & OtherIons & $32 \mathrm{~S} \times 64 \mathrm{E} \times 21 \mathrm{~A}$ & 1376 & $36 \mathrm{~V} \times 64 \mathrm{E}$ & 37 & $64 \mathrm{~s}$ & $256 \mathrm{~s}$ \\
\hline M15 & OtherIons & $32 \mathrm{~S} \times 64 \mathrm{E} \times 21 \mathrm{~A}$ & 1376 & $36 \mathrm{~V} \times 64 \mathrm{E}$ & 37 & $64 \mathrm{~s}$ & $256 \mathrm{~s}$ \\
\hline TSTC & STC TOF & $64 \mathrm{E} \times 1024 \mathrm{~T}$ & 2097 & $32 \mathrm{E} \times 1024 \mathrm{~T}$ & 524 & $64 \mathrm{~s}$ & $256 \mathrm{~s}$ \\
\hline TSTE & STE TOF & $64 \mathrm{E} \times 1024 \mathrm{~T}$ & 2097 & $32 \mathrm{E} \times 1024 \mathrm{~T}$ & 524 & $64 \mathrm{~s}$ & $256 \mathrm{~s}$ \\
\hline TLEF & LEF TOF & $64 \mathrm{E} \times 2048 \mathrm{~T}$ & 4194 & $8 \mathrm{E} \times 2048 \mathrm{~T}$ & 262 & $64 \mathrm{~s}$ & $256 \mathrm{~s}$ \\
\hline SUM & & & 27652 & & 1913 & & \\
\hline
\end{tabular}

produced via charge exchange during crossing of the carbon foil (e.g., Funsten et al. 2001). Using this mass identification, the moments are subsequently calculated by MSA DPU (assuming $\mathrm{Na}$ mass for heavy ions because this latter group of ions corresponds to all ions with $\mathrm{m} / \mathrm{q}$ ratios larger than 2 ).

MSA internal products thus contain two types of count rate matrices: (i) TOF matrices with no angular information for LEF data, and (ii) mass matrices as described above for ST data. These internal matrices are then transformed to external matrices for telemetry. In the latter matrices, energy sampling is reduced to eight steps in the LEF data. For ST data, angular resolution is reduced to 36 view directions per spin. These external matrices are then transmitted according to different modes selected on a scientific basis; normal mode (default mode) as described in Table 15, high angular resolution mode (Table 16), and high time resolution mode (Table 17). Other modes (not shown) include event mode and burst mode (similar to Table 17 but with a higher production frequency).

The data downlink includes three distinct telemetry rates for Mio data: L-mode (L) available most of the time, M-mode (M) during $~ 25 \%$ of the time, and $\mathrm{H}$-mode $(\mathrm{H})$ in some very limited portions of the Mio orbit. MSA is part of the MPPE particle consortium; thus, the data modes are defined for specific MPPE science research targets (e.g., survey mode, solar wind or exosphere dedicated modes, reconnection mode) which is consistent with the modes adopted for the other particle instruments. Table 18 shows the MSA data that will 
Table 16 MSA high spatial resolution mode

\begin{tabular}{|c|c|c|c|c|c|c|c|}
\hline \multirow[t]{2}{*}{ Name } & \multirow[t]{2}{*}{ Content } & \multicolumn{2}{|l|}{ Internal (32 bit) } & \multicolumn{2}{|l|}{ External (16 bit) } & \multicolumn{2}{|c|}{ Time resolution } \\
\hline & & Dimension & kbit & Dimension & kbit & Int & Med \\
\hline M0 & Starts & $32 \mathrm{~S} \times 32 \mathrm{E} \times 21 \mathrm{~A}$ & 688 & $21 \mathrm{~A} \times 32 \mathrm{~S} \times 2 \mathrm{E}$ & 21 & $4 \mathrm{~s}$ & $16 \mathrm{~s}$ \\
\hline M1 & Protons & $32 \mathrm{~S} \times 32 \mathrm{E} \times 21 \mathrm{~A}$ & 688 & $21 \mathrm{~A} \times 32 \mathrm{~S} \times 4 \mathrm{E}$ & 43 & $4 \mathrm{~s}$ & $16 \mathrm{~s}$ \\
\hline M2 & $\mathrm{He}^{++}$ & $32 \mathrm{~S} \times 32 \mathrm{E} \times 21 \mathrm{~A}$ & 688 & $21 \mathrm{~A} \times 32 \mathrm{~S} \times 4 \mathrm{E}$ & 43 & $4 \mathrm{~s}$ & $48 \mathrm{~s}$ \\
\hline M3 & HeavyIons & $32 \mathrm{~S} \times 32 \mathrm{E} \times 21 \mathrm{~A}$ & 688 & $21 \mathrm{~A} \times 32 \mathrm{~S} \times 4 \mathrm{E}$ & 43 & & $48 \mathrm{~s}$ \\
\hline M4 & OtherIons & $32 \mathrm{~S} \times 64 \mathrm{E} \times 21 \mathrm{~A}$ & 1376 & $21 \mathrm{~A} \times 32 \mathrm{~S} \times 4 \mathrm{E}$ & 43 & $64 \mathrm{~s}$ & $256 \mathrm{~s}$ \\
\hline M5 & OtherIons & $32 \mathrm{~S} \times 64 \mathrm{E} \times 21 \mathrm{~A}$ & 1376 & $21 \mathrm{~A} \times 32 \mathrm{~S} \times 4 \mathrm{E}$ & 43 & $64 \mathrm{~s}$ & $256 \mathrm{~s}$ \\
\hline M6 & OtherIons & $32 \mathrm{~S} \times 64 \mathrm{E} \times 21 \mathrm{~A}$ & 1376 & $21 \mathrm{~A} \times 32 \mathrm{~S} \times 4 \mathrm{E}$ & 43 & $64 \mathrm{~s}$ & $256 \mathrm{~s}$ \\
\hline M7 & OtherIons & $32 \mathrm{~S} \times 64 \mathrm{E} \times 21 \mathrm{~A}$ & 1376 & $21 \mathrm{~A} \times 32 \mathrm{~S} \times 4 \mathrm{E}$ & 43 & $64 \mathrm{~s}$ & $256 \mathrm{~s}$ \\
\hline M8 & OtherIons & $32 \mathrm{~S} \times 64 \mathrm{E} \times 21 \mathrm{~A}$ & 1376 & $21 \mathrm{~A} \times 32 \mathrm{~S} \times 4 \mathrm{E}$ & 43 & $64 s$ & $256 \mathrm{~s}$ \\
\hline M9 & OtherIons & $32 \mathrm{~S} \times 64 \mathrm{E} \times 21 \mathrm{~A}$ & 1376 & $21 \mathrm{~A} \times 32 \mathrm{~S} \times 4 \mathrm{E}$ & 43 & $64 \mathrm{~s}$ & $256 \mathrm{~s}$ \\
\hline M10 & OtherIons & $32 \mathrm{~S} \times 64 \mathrm{E} \times 21 \mathrm{~A}$ & 1376 & $21 \mathrm{~A} \times 32 \mathrm{~S} \times 4 \mathrm{E}$ & 43 & $64 \mathrm{~s}$ & $256 \mathrm{~s}$ \\
\hline M11 & OtherIons & $32 \mathrm{~S} \times 64 \mathrm{E} \times 21 \mathrm{~A}$ & 1376 & $21 \mathrm{~A} \times 32 \mathrm{~S} \times 4 \mathrm{E}$ & 43 & $64 \mathrm{~s}$ & $256 \mathrm{~s}$ \\
\hline M12 & OtherIons & $32 \mathrm{~S} \times 64 \mathrm{E} \times 21 \mathrm{~A}$ & 1376 & $21 \mathrm{~A} \times 32 \mathrm{~S} \times 4 \mathrm{E}$ & 43 & $64 \mathrm{~s}$ & $256 \mathrm{~s}$ \\
\hline M13 & OtherIons & $32 \mathrm{~S} \times 64 \mathrm{E} \times 21 \mathrm{~A}$ & 1376 & $21 \mathrm{~A} \times 32 \mathrm{~S} \times 4 \mathrm{E}$ & 43 & $64 \mathrm{~s}$ & $256 \mathrm{~s}$ \\
\hline M14 & OtherIons & $32 \mathrm{~S} \times 64 \mathrm{E} \times 21 \mathrm{~A}$ & 1376 & $21 \mathrm{~A} \times 32 \mathrm{~S} \times 4 \mathrm{E}$ & 43 & $64 \mathrm{~s}$ & $256 \mathrm{~s}$ \\
\hline M15 & OtherIons & $32 \mathrm{~S} \times 64 \mathrm{E} \times 21 \mathrm{~A}$ & 1376 & $21 \mathrm{~A} \times 32 \mathrm{~S} \times 4 \mathrm{E}$ & 43 & $64 \mathrm{~s}$ & $256 \mathrm{~s}$ \\
\hline TSTC & STC TOF & $64 \mathrm{E} \times 1024 \mathrm{~T}$ & 2097 & $32 \mathrm{E} \times 1024 \mathrm{~T}$ & 524 & $64 s$ & $256 \mathrm{~s}$ \\
\hline TSTE & STE TOF & $64 \mathrm{E} \times 1024 \mathrm{~T}$ & 2097 & $32 \mathrm{E} \times 1024 \mathrm{~T}$ & 524 & $64 \mathrm{~s}$ & $256 \mathrm{~s}$ \\
\hline TLEF & LEF TOF & $64 \mathrm{E} \times 2048 \mathrm{~T}$ & 4194 & $8 \mathrm{E} \times 2048 \mathrm{~T}$ & 262 & $64 \mathrm{~s}$ & $256 \mathrm{~s}$ \\
\hline SUM & & & 27652 & & 1976 & & \\
\hline
\end{tabular}

be transmitted in the various Mio telemetry regimes depending upon the targeted research (Medium A for survey, Medium C for solar wind or exosphere analysis). Since the MSA possesses a CPU board that processes the mission data into the mission products such as moment data, the MSA application in the MDP1 relays them to the data recorder. The MSA application removes the dummy data and packs only effective data into the mission packets.

\subsubsection{Pre-flight Calibration of MSA}

Because the MSA instrument is a collaborative effort between four different teams, both the Flight Model and the Spare Model were assembled at the PI institute (ISAS, Sagamihara, Japan). Numerous test campaigns including pre-flight calibration were subsequently conducted at this institute. The present section provides a summary of the calibration results.

Downstream of the MSA entrance, ions travel through the spherical energy analyser, which has an external electrode at ground potential. On the inner electrode of the analyzer, the voltage applied varies up to a maximum value of $-5.6 \mathrm{kV}$, which enables selection of ions with specific energy per charge (E/q) ratios. This is illustrated in Fig. 48, which shows

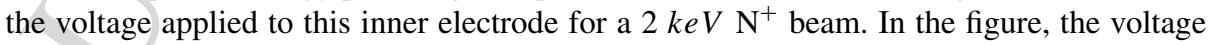
median value is approximately $-292 \mathrm{~V}$, yielding an analyzer constant $\mathrm{k}$ of $\sim 6.85$. With minimum and maximum voltages of $-0.2 \mathrm{~V}$ and $-5.6 \mathrm{kV}$, respectively, MSA can thus operate from a few electron volts per charge (eV/q) up to $\sim 38 \mathrm{keV} / \mathrm{q}$. The figure also shows a modulation of the ion count rate within each of the 21 entrance windows owing to the partition walls at each $11.25^{\circ}$ step. Further analysis of the energy analyzer response revealed 
Table 17 MSA high time resolution mode

\begin{tabular}{|c|c|c|c|c|c|c|c|}
\hline \multirow[t]{2}{*}{ Name } & \multirow[t]{2}{*}{ Content } & \multicolumn{2}{|l|}{ Internal (32 bit) } & \multicolumn{2}{|c|}{ External (16 bit) } & \multicolumn{2}{|c|}{ Time resolution } \\
\hline & & Dimension & kbit & Dimension & kbit & Int & Med \\
\hline M0 & Starts & $32 \mathrm{~S} \times 32 \mathrm{E} \times 21 \mathrm{~A}$ & 688 & $36 \mathrm{~V} \times 32 \mathrm{E}$ & 18 & $4 \mathrm{~s}$ & $8 \mathrm{~s}$ \\
\hline A0 & Starts & & & $21 \mathrm{~A} \times 32 \mathrm{~S}$ & 11 & $64 s$ & $64 s$ \\
\hline M1 & Protons & $32 \mathrm{~S} \times 32 \mathrm{E} \times 21 \mathrm{~A}$ & 688 & $36 \mathrm{~V} \times 32 \mathrm{E}$ & 18 & $4 \mathrm{~s}$ & $8 \mathrm{~s}$ \\
\hline M2 & $\mathrm{He}++$ & $32 \mathrm{~S} \times 32 \mathrm{E} \times 21 \mathrm{~A}$ & 688 & $36 \mathrm{~V} \times 16 \mathrm{E}$ & 9 & & $12 \mathrm{~s}$ \\
\hline M3 & HeavyIons & $32 \mathrm{~S} \times 32 \mathrm{E} \times 21 \mathrm{~A}$ & 688 & $36 \mathrm{~V} \times 16 \mathrm{E}$ & 9 & $4 \mathrm{~s}$ & $12 \mathrm{~s}$ \\
\hline M4 & OtherIons & $32 \mathrm{~S} \times 64 \mathrm{E} \times 21 \mathrm{~A}$ & 1376 & $36 \mathrm{~V} \times 16 \mathrm{E}$ & 9 & $64 s$ & $64 \mathrm{~s}$ \\
\hline M5 & OtherIons & $32 \mathrm{~S} \times 64 \mathrm{E} \times 21 \mathrm{~A}$ & 1376 & $36 \mathrm{~V} \times 16 \mathrm{E}$ & 9 & $64 s$ & $64 s$ \\
\hline M6 & OtherIons & $32 \mathrm{~S} \times 64 \mathrm{E} \times 21 \mathrm{~A}$ & 1376 & $36 \mathrm{~V} \times 16 \mathrm{E}$ & 9 & $64 \mathrm{~s}$ & $64 \mathrm{~s}$ \\
\hline M7 & OtherIons & $32 \mathrm{~S} \times 64 \mathrm{E} \times 21 \mathrm{~A}$ & 1376 & $36 \mathrm{~V} \times 16 \mathrm{E}$ & 9 & $64 \mathrm{~s}$ & $64 s$ \\
\hline M8 & OtherIons & $32 \mathrm{~S} \times 64 \mathrm{E} \times 21 \mathrm{~A}$ & 1376 & $36 \mathrm{~V} \times 16 \mathrm{E}$ & 9 & $64 \mathrm{~s}$ & $64 s$ \\
\hline M9 & OtherIons & $32 \mathrm{~S} \times 64 \mathrm{E} \times 21 \mathrm{~A}$ & 1376 & $36 \mathrm{~V} \times 16 \mathrm{E}$ & 9 & $64 s$ & $64 \mathrm{~s}$ \\
\hline M10 & OtherIons & $32 \mathrm{~S} \times 64 \mathrm{E} \times 21 \mathrm{~A}$ & 1376 & $36 \mathrm{~V} \times 16 \mathrm{E}$ & 9 & $64 \mathrm{~s}$ & $64 \mathrm{~s}$ \\
\hline M11 & OtherIons & $32 \mathrm{~S} \times 64 \mathrm{E} \times 21 \mathrm{~A}$ & 1376 & $36 \mathrm{~V} \times 16 \mathrm{E}$ & 9 & $64 \mathrm{~s}$ & $64 \mathrm{~s}$ \\
\hline M12 & OtherIons & $32 \mathrm{~S} \times 64 \mathrm{E} \times 21 \mathrm{~A}$ & 1376 & $36 \mathrm{~V} \times 16 \mathrm{E}$ & 9 & $64 \mathrm{~s}$ & $64 s$ \\
\hline M13 & OtherIons & $32 \mathrm{~S} \times 64 \mathrm{E} \times 21 \mathrm{~A}$ & 1376 & $36 \mathrm{~V} \times 16 \mathrm{E}$ & 9 & $64 \mathrm{~s}$ & $64 \mathrm{~s}$ \\
\hline M14 & OtherIons & $32 \mathrm{~S} \times 64 \mathrm{E} \times 21 \mathrm{~A}$ & 1376 & $36 \mathrm{~V} \times 16 \mathrm{E}$ & 9 & $64 s$ & $64 s$ \\
\hline M15 & OtherIons & $32 \mathrm{~S} \times 64 \mathrm{E} \times 21 \mathrm{~A}$ & 1376 & $36 \mathrm{~V} \times 16 \mathrm{E}$ & 9 & $64 \mathrm{~s}$ & $64 \mathrm{~s}$ \\
\hline TSTC & STC TOF & $64 \mathrm{E} \times 1024 \mathrm{~T}$ & 2097 & $4 \mathrm{E} \times 1024 \mathrm{~T}$ & 65 & $64 \mathrm{~s}$ & $64 \mathrm{~s}$ \\
\hline TSTE & STE TOF & $64 \mathrm{E} \times 1024 \mathrm{~T}$ & 2097 & $4 \mathrm{E} \times 1024 \mathrm{~T}$ & 65 & $64 \mathrm{~s}$ & $64 \mathrm{~s}$ \\
\hline TLEF & LEF TOF & $64 \mathrm{E} \times 2048 \mathrm{~T}$ & & $2 \mathrm{E} \times 2048 \mathrm{~T}$ & 65 & $64 \mathrm{~s}$ & $64 s$ \\
\hline SUM & & & 27652 & & 368 & & \\
\hline
\end{tabular}

Fig. 48 Color-coded count rate as functions of azimuth and voltage applied on the inner sphere of the energy analyzer for a $2 \mathrm{keV} \mathrm{N}^{+}$beam

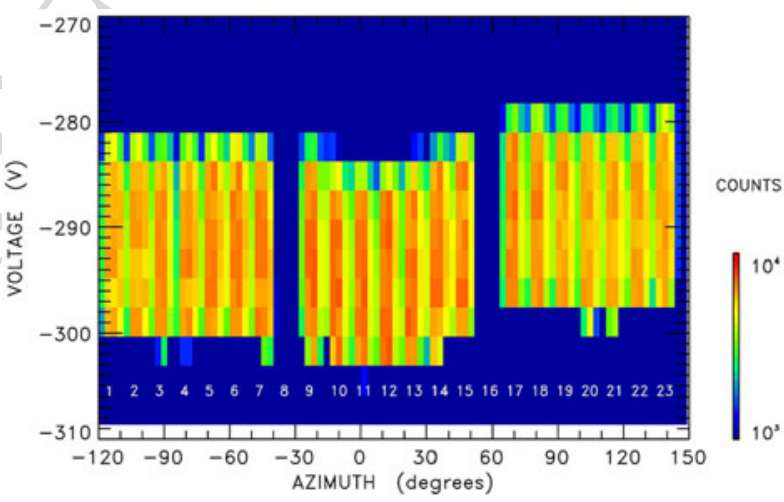

energy resolution of $\sim 8.5 \%$. Finally, the figure shows the MSA response as a function of azimuth or, equivalently, the entrance windows. In the other dimension (i.e., elevation or polar angle), the MSA FOV is centered at $\sim 5^{\circ}$ with an angular resolution (full width at half maximum, FWHM) of $\sim 5^{\circ}$. This is illustrated in Fig. 49 , which shows particle counts versus voltage and polar angle (elevation).

At exit of the energy analyzer, ions are accelerated toward the entrance of the TOF chamber with nominal operation voltages of $\pm 11 \mathrm{kV}$ corresponding to $85 \%$ of the MSA quali- 
"SPAC 11214 layout: Small Condensed file: spac839.tex (karolis.kavaliauskas) class: spr-small-v1 2020/06/02 v2.07 Prn:22/07/2021; 13:21 p. 47/91" doctopic: ReviewPaper numbering style: ContentOnly reference style: aps"

MPPE on MMO (Mio)

Page 47 of 91

\#\#\#\#_-

2301

2302

2303

2304

2305

2306

2307

2308

2309

2310

2311

2312

2313

2314

2315

2316

2317

2318

2319

2320

2321

2322

2323

2324

2325

2326

2327

2328

2329

2330

2331

2332

2333

2334

2335

2336

2337

2338

2339

2340

2341

2342

2343

2344

2345

2346

2347

2348

2349

2350

Table 18 MSA data for the different Mio telemetry regimes

\begin{tabular}{|c|c|c|c|c|c|c|}
\hline \multirow[t]{2}{*}{ Mode } & \multirow[t]{2}{*}{ Products } & \multirow[t]{2}{*}{ bits/spin } & \multirow[t]{2}{*}{ bits/64 s } & \multicolumn{3}{|l|}{ Compressed } \\
\hline & & & & /64 s(TBC) & /spin & $/ \mathrm{s}$ \\
\hline \multirow[t]{4}{*}{ Low } & P0L-P3L, SP & 357 & 5712 & 5712 & & \\
\hline & M1L-M3L & 192 & 3072 & 300 (L10) & & \\
\hline & TSTL & 2048 & 32768 & 3300 (LL-10) & & \\
\hline & DataOut & & & 9312 & 582 & 145 \\
\hline \multirow[t]{10}{*}{ Med A } & $\mathrm{P} 0-\mathrm{P} 3, \mathrm{SP}$ & 645 & 10320 & 10320 & & \\
\hline & P4-P15 & 60 & 960 & 960 & & \\
\hline & M0, M1 & 12288 & 196608 & 19700 (L10) & & \\
\hline & A0 & 184 & 2944 & 290 (L10) & & \\
\hline & M2, M3 & 6144 & 98304 & 9800 (L10) & & \\
\hline & M4-M15 & 7135 & 114160 & $11400(\mathrm{~L} 10)$ & & \\
\hline & TSTC & & 131072 & 13100 (LL-10) & & \\
\hline & TSTE & & 131072 & 13100 (LL-10) & & \\
\hline & TLEF & & 65536 & $6500(\mathrm{LL}-10)$ & & \\
\hline & DataOut & & & 85190 & 5324 & 1331 \\
\hline \multirow[t]{9}{*}{ Med C } & P0-P3, SP & 645 & 10320 & 10320 & & \\
\hline & P4-P15 & 60 & 960 & 960 & & \\
\hline & M0, M1 & 17664 & 282624 & $28300(\mathrm{~L} 10)$ & & \\
\hline & $\mathrm{M} 2, \mathrm{M} 3$ & 8832 & 141312 & 14100 (L10) & & \\
\hline & M4-M15 & 8832 & 141312 & $14100(\mathrm{~L} 10)$ & & \\
\hline & TSTC & & 131072 & 13100 (LL-10) & & \\
\hline & TSTE & & 131072 & 13100 (LL-10) & & \\
\hline & TLEF & & 65536 & 6500 (LL-10) & & \\
\hline & DataOut & & & 100480 & 6280 & 1570 \\
\hline \multirow[t]{10}{*}{ Med D } & $\mathrm{P} 0-\mathrm{P} 3, \mathrm{SP}$ & 645 & 10320 & 10320 & & \\
\hline & P4-P15 & 60 & 960 & 960 & & \\
\hline & M0, M1 & 18432 & 294912 & 29500 (L10) & & \\
\hline & $\mathrm{A} 0$ & 736 & 11776 & 1200 (L10) & & \\
\hline & M2, M3 & 6144 & 98304 & 9800 (L10) & & \\
\hline & M4-M15 & 7135 & 114160 & 11400 (L10) & & \\
\hline & TSTC & & 131072 & 13100 (LL-10) & & \\
\hline & TSTE & & 131072 & 13100 (LL-10) & & \\
\hline & TLEF & & 65536 & 6500 (LL-10) & & \\
\hline & DataOut & & & 96280 & 6017 & 1504 \\
\hline \multirow[t]{7}{*}{ Med E } & P0-P3, SP & 645 & 10320 & 10320 & & \\
\hline & P4-P15 & 60 & 960 & 960 & & \\
\hline & M0, M1 & 14592 & 233472 & 23500 (L10) & & \\
\hline & M2, M3 & 6144 & 98304 & 9800 (L10) & & \\
\hline & TLEF & & 69905 & 3500 (L20) & & \\
\hline & Events & 2618 & 41900 & 41900 & & \\
\hline & DataOut & & & 86480 & 5405 & 1351 \\
\hline
\end{tabular}



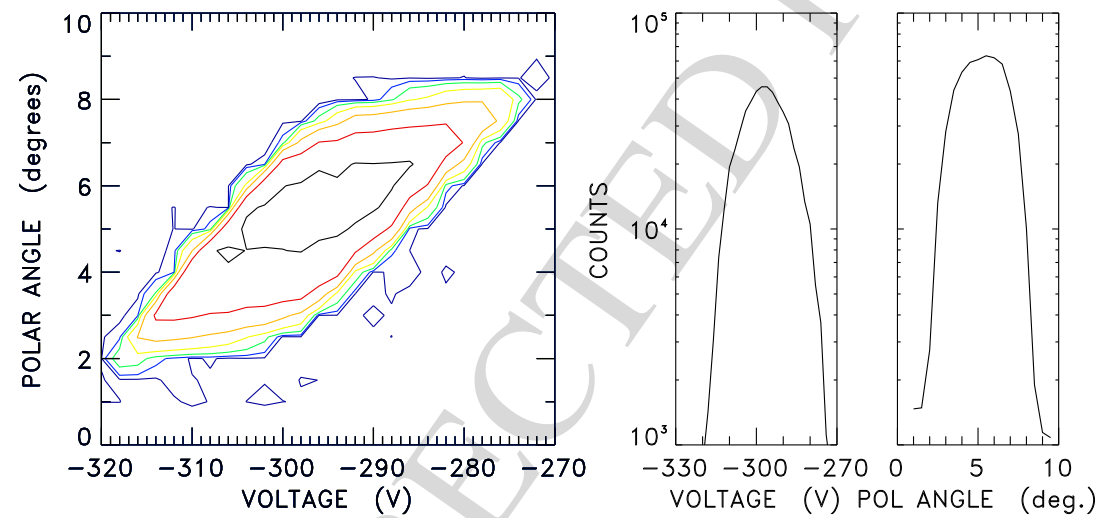

Fig. 49 Count rate contours as functions of the polar angle (elevation) and voltage (energy) for MSA entrance window No. 11 using a $2 \mathrm{keV} \mathrm{N}^{+}$beam (left). Integrated counts versus voltage and integrated counts versus elevation (right) (after Delcourt et al. 2016)

fication voltages; the maximum operation voltages are $\pm 12 \mathrm{kV}$. Notably a grid, effectively acting as an electrostatic lens polarized at $1 \mathrm{kV}$ located near the exit of the energy analyzer prevents the large accelerating electric field from penetrating deep into this analyzer and has a detrimental effect on ion trajectories. Upon entry to the TOF chamber, ions interact with thin $\left(\sim 1 \mu \mathrm{g} / \mathrm{cm}^{2}\right)$ carbon foils, which leads to emission of one or several secondary electrons in both forward and backward directions. With the help of an electrode tailored for this purpose, forward electrons are deflected toward the outer part of the LEF MCP (Fig. 39). At the back of this MCP, electrons are collected on a delay line and the START pulse obtained is used to trigger TOF measurement. That is, the START pulse opens a TOF window of $\sim 1560$ ns during which a STOP pulse is expected; in practice, up to three STOP pulses can be recorded. Moreover, the position of the electron impact on the delay line provides information on the azimuthal sector of the incoming ion. This is illustrated in Fig. 50, where the color map shows data obtained between the 60 delay line sectors and the 21 entrance windows (or azimuth). Similar to that shown in Fig. 48, the modulation of the ion count rate is 
Fig. 50 Color-coded count rate as functions of azimuth and delay line sector for a $2 \mathrm{keV} \mathrm{N}^{+}$beam

attributed to the regularly spaced partition walls at the entrance of the instrument. Measurements of the START rate in the different delay line sectors yield the 3D angular and energy distributions of the ions without mass identification but with high temporal resolution.

To protect MCP from the potentially intense solar wind flux, a "spoiler" capability has been included in the design of the energy analyzer. As previously mentioned, the external electrode of this analyzer has been designed in three mechanical parts, with the capability to polarize the central one independently from the other two for de-focusing or spoiling the incoming ion beam. When the ion count rate over one spin exceeds $10^{3}$ START events in one energy bin and one entrance window, the voltage on the central electrode is increased by one step at the end of the spin. Conversely, when the count rate drops below $5 \times 10^{2}$ START events, the spoiler voltage is decreased by one step. At the first switch-on of the spoiler, the voltage value used is that corresponding to $10 \%$ efficiency $(56 \%$ of the inner electrode voltage) to effectively protect the MCP. Subsequently, the spoiler voltage is further increased (decreased) if the count rate is excessively high (low).

After crossing the carbon foils, the ions travel inside the TOF chamber and impact either the LEF MCP or ST MCP depending on their charge state; hence, a STOP pulse can be associated with the corresponding START to derive the particle TOF and its $\mathrm{m} / \mathrm{q}$ ratio. As previously mentioned, LEF data are characterized by low count rates owing to the small fraction of ions that remain positively charged after crossing the carbon foils but a high mass resolution, which is of primary interest for planetology science. In contrast, ST data are characterized by high count rates (owing to the large fraction of ions neutralized during carbon foil crossing) but lower mass resolution, although this mass resolution can be improved to some degree by considering a small collection area at the center of the ST MCP. An example of the TOF data obtained is provided in Fig. 51. The top panel in the figure shows LEF data for different ion species of $\mathrm{N}^{+}, \mathrm{O}^{+}, \mathrm{Na}^{+}$and $\mathrm{K}^{+}$. Notably, the width of the measured spectra is narrow regardless of the ion mass. The bottom panel of the figure shows ST data for conditions similar to those in the top panel. These ST spectra, which resemble those of MESSENGER FIPS, clearly contrast with those of LEF (top panel) with larger count rates and much lower mass resolution. In particular, the spectrum achieved for $\mathrm{K}^{+}$ions that spreads over a large TOF interval, owing to large angular diffusion and energy straggling upon crossing the carbon foils, cannot be clearly identified in the long TOF tail. As expected, the center panel of Fig. 51 that shows the TOF spectra obtained on the central ST exhibits a somewhat enhanced mass resolution and lower count rates. Moreover, different ghost peaks appearing in the figure are attributed to secondary emissions inside the TOF 


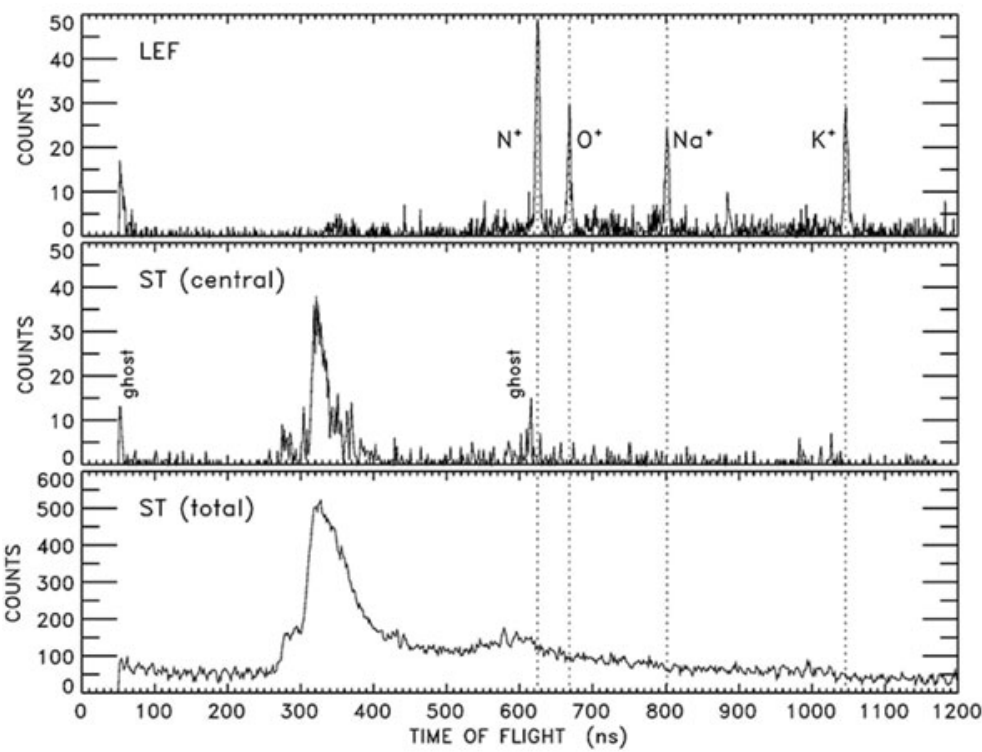

Fig. 51 TOF spectra for a $5 \mathrm{keV}$ beam of $\mathrm{N}^{+}, \mathrm{O}^{+}, \mathrm{Na}^{+}$and $\mathrm{K}^{+}$ions with TOF chamber high voltage set to $\pm 11 \mathrm{kV}$. From top to bottom: LEF, central ST and total ST (after Delcourt et al. 2016)

chamber (Fig. 10 of Delcourt et al. 2016). Generally, these ghost peaks are less pronounced at larger operation voltages.

Tables 19 and 20 provide a more quantitative view of the results obtained from TOF chamber calibration. As an example, Table 19 shows the TOF parameters obtained for two different energies and two different operation voltages for $\mathrm{He}^{+}, \mathrm{Na}^{+}$and $\mathrm{K}^{+}$ions. For a given operation voltage $( \pm 8 \mathrm{kV}$ or $\pm 11 \mathrm{kV})$, the isochronous nature of the LEF spectra is clearly apparent with similar $\mathrm{T}_{m}$ values regardless of the ion energy. In addition, the narrow width of the spectra led to enhanced mass resolution (computed as $\mathrm{T}_{m} / \Delta \mathrm{T}$ ) of at least 100 .

In contrast to that in Table 19, the ST results in Table 20 clearly exhibit $\mathrm{T}_{m}$ values that decrease with increasing energy, regardless of ion species $\left(\mathrm{He}^{+}\right.$or $\left.\mathrm{K}^{+}\right)$or operation voltage $( \pm 8$ or $\pm 11 \mathrm{kV})$. Moreover, the TOF spectra width increased significantly for $\mathrm{K}^{+}$, exemplifying the poor mass resolution capability of ST for heavy ions.

A global view of the MSA TOF-mass mapping is provided in Fig. 52, which shows the TOF intervals used to identify the ion species depending upon their energy.

\subsubsection{Near-Earth Commissioning Results of MSA}

In contrast to MPO, the Mio spacecraft will spin (4 s) during the orbit phase at Mercury. During the seven-year cruise, Mio is hidden behind the MOSIF thermal shield to avoid harsh solar radiation. As a result, MPPE particle instruments have narrow FOVs pointing along the Mio spin axis; thus, very limited data are expected throughout the cruise phase. In contrast to electrons that are nearly isotropic, the highly collimated solar wind ions cannot be recorded in the cruise phase configuration, because the solar wind direction is obstructed by MOSIF; only dark counts can be obtained. To simulate ions entering the energy analyzer, MSA features a calibration pulse that can be parameterized with different frequencies and delay line sectors. This START-like calibration pulse was used during near-Earth commissioning to check the MSA status and data flow. 
"SPAC 11214 layout: Small Condensed file: spac839.tex (karolis.kavaliauskas) class: spr-small-v1 2020/06/02 v2.07 Prn:22/07/2021; 13:21 p. 51/91" doctopic: ReviewPaper numbering style: ContentOnly reference style: aps"

MPPE on MMO (Mio)

Page 51 of 91

\#\#\#\#

2501

2502

2503

2504

2505

2506

2507

2508

2509

2510

2511

2512

2513

2514

2515

2516

2517

2518

2519

2520

2521

2522

2523

2524

2525

2526

2527

2528

2529

2530

2531

2532

2533

2534

2535

2536

2537

2538

2539

2540

2541

2542

2543

2544

2545

2546

2547

2548

2549

2550
Table 19 Selected parameters of MSA LEF spectra. The mass resolution is derived from $\mathrm{Tm} / \Delta \mathrm{T}$ where $\mathrm{Tm}$ is the median value and $\Delta \mathrm{T}$ is the TOF spectra FWHM

Table 20 Sam as Table 19 but for central ST and total ST

\begin{tabular}{lllll}
\hline $\mathrm{He}^{+}$ & & & & \\
\hline TOF voltage & Energy & $\mathrm{Tm}(\mathrm{ns})$ & $\Delta \mathrm{T}(\mathrm{ns})$ & $\mathrm{Tm} / \Delta \mathrm{T}$ \\
\hline $\pm 8 \mathrm{kV}$ & $1-2 \mathrm{keV}$ & 398.1 & 27.1 & 14.7 \\
& $4-6 \mathrm{keV}$ & 394.9 & 33.4 & 11.8 \\
$\pm 11 \mathrm{kV}$ & $1-2 \mathrm{keV}$ & 336.3 & 5.5 & 61.4 \\
& $4-6 \mathrm{keV}$ & 335.2 & 27.2 & 12.3 \\
\hline $\mathrm{Na}^{+}$ & & & & \\
\hline $\mathrm{TOF}$ voltage & Energy & $\mathrm{Tm}(\mathrm{ns})$ & $\Delta \mathrm{T}(\mathrm{ns})$ & $\mathrm{Tm} / \Delta \mathrm{T}$ \\
\hline $\pm 8 \mathrm{kV}$ & $1-2 \mathrm{keV}$ & 957.6 & 5.8 & 166.0 \\
& $4-6 \mathrm{keV}$ & 955.4 & 4.3 & 222.4 \\
$\pm 11 \mathrm{kV}$ & $1-2 \mathrm{keV}$ & 810.7 & 6.8 & 118.7 \\
& $4-6 \mathrm{keV}$ & 809.7 & 4.7 & 172.7 \\
\hline $\mathrm{K}^{+}$ & & & & \\
\hline TOF voltage & Energy & $\mathrm{Tm}(\mathrm{ns})$ & $\Delta \mathrm{T}(\mathrm{ns})$ & $\mathrm{Tm} / \Delta \mathrm{T}$ \\
\hline $\pm 8 \mathrm{kV}$ & $1-2 \mathrm{keV}$ & 1249.4 & 11.1 & 112.5 \\
$\pm 11 \mathrm{kV}$ & $4-6 \mathrm{keV}$ & 1247.2 & 11.2 & 111.1 \\
& $1-2 \mathrm{keV}$ & 1057.5 & 7.2 & 146.5 \\
$1-6 \mathrm{keV}$ & 1055.5 & 9.3 & 113.3 \\
\hline
\end{tabular}

\begin{tabular}{llllll}
\hline $\mathrm{He}^{+}$ & & & & & \\
\hline TOF yoltage & ST & Energy & Tm $(\mathrm{ns})$ & $\Delta \mathrm{T}(\mathrm{ns})$ & $\mathrm{Tm} / \Delta \mathrm{T}$ \\
\hline $\pm 8 \mathrm{kV}$ & \multirow{2}{*}{ central } & $4 \mathrm{keV}$ & 193.7 & 8.6 & 22.4 \\
& & $10 \mathrm{keV}$ & 154.9 & 7.8 & 19.8 \\
& \multirow{2}{*}{ total } & $4 \mathrm{keV}$ & 195.5 & 14.1 & 13.9 \\
& & $10 \mathrm{keV}$ & 158.2 & 12.8 & 12.3 \\
& \multirow{3}{*}{ central } & $4 \mathrm{keV}$ & 171.3 & 7.6 & 22.5 \\
& & $10 \mathrm{keV}$ & 142.5 & 7.4 & 19.4 \\
& \multirow{3}{*}{ total } & $4 \mathrm{keV}$ & 173.2 & 12.4 & 14.0 \\
& & $10 \mathrm{keV}$ & 144.6 & 9.6 & 15.0 \\
\hline
\end{tabular}

\begin{tabular}{llllll}
\hline $\mathrm{K}^{+}$ & & & & & \\
\hline TOF voltage & ST & Energy & Tm (ns) & $\Delta \mathrm{T}(\mathrm{ns})$ & $\mathrm{Tm} / \Delta \mathrm{T}$ \\
\hline $\pm 8 \mathrm{kV}$ & \multirow{2}{*}{ central } & $4 \mathrm{keV}$ & 662.5 & 322.7 & 2.1 \\
& & $10 \mathrm{keV}$ & 581.3 & 342.2 & 1.7 \\
& \multirow{2}{*}{ total } & $4 \mathrm{keV}$ & 681.8 & 261.4 & 2.4 \\
& & $10 \mathrm{keV}$ & 609.5 & 268.2 & 2.3 \\
$\pm 11 \mathrm{kV}$ & \multirow{2}{*}{ central } & $4 \mathrm{keV}$ & 620.4 & 173.0 & 3.6 \\
& & $10 \mathrm{keV}$ & 525.0 & 116.1 & 4.5 \\
& \multirow{3}{*}{ total } & $4 \mathrm{keV}$ & 644.7 & 236.7 & 2.7 \\
& & $10 \mathrm{keV}$ & 556.5 & 251.1 & 2.2 \\
\hline
\end{tabular}




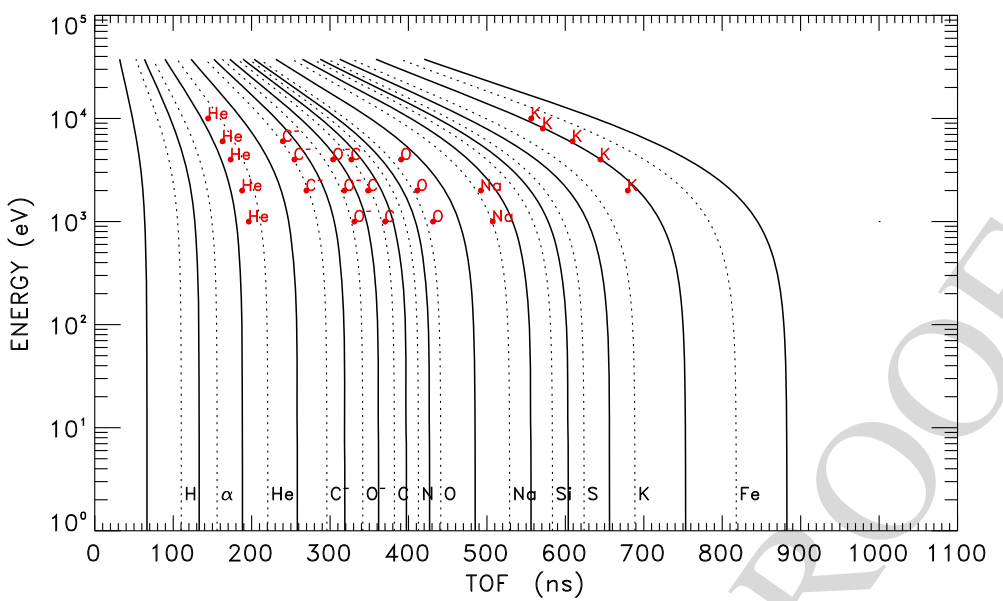

Fig. 52 TOF variation versus energy for different ion species (dotted lines) as obtained from equation (3) of Delcourt et al. (2016) with $\mathrm{L}=16 \mathrm{~cm}$. The solid lines depict the TOF intervals attributed to each species and the red dots show the results of MSA FM calibration. The TOF chamber voltage is set to $11 \mathrm{kV}$

Similar to that for other MPPE instruments, near-Earth commissioning of MSA has been organized in two sequences, with the first in November 2018 to check basic functionalities, and the second in June 2019 to check high voltages. Owing to the transmission of data via the MPO spacecraft during the cruise, only the L-mode telemetry regime is available. A problem was encountered during the first MSA commissioning sequence that appeared nominal until a calibration pulse triggered was not followed by L-mode data reception. Debriefing was performed and possible causes including hardware failure were explored according to fault tree analysis scheme. The second MSA commissioning sequence provided new information on this problem because the L-mode data acquisition was successful in June 2019 but was contingent upon rebooting of MDP1, the MPPE dedicated DPU, before operating MSA. Although this rules out a hardware failure, the reason for this faulty behavior is not yet understood and still is under investigation.

Throughout the commissioning and operations, voltages are monitored through MSA dedicated CPUs via comparison of HK values with the command values. If both values are not consistent (with 15\% margin) after one spin, an error message is produced in the corresponding Mission Data packet, if such an error message is obtained during four consecutive spins, an emergency MSA shutdown is issued. HV monitoring is initiated via upload of the On-board Command Language (OCL) procedure from EEPROM. When this HV monitoring is correctly enabled, the HK return value is "ON". Prior to HV setup, nine commands are sent to enable measurements and to set thresholds for START and STOP signals. The HV setup procedure is then conducted according to six different procedures corresponding to $\pm 8 \mathrm{kV}, \pm 10 \mathrm{kV}$ and $\pm 11 \mathrm{kV}$ in normal or safe modes with execution times varying among the procedures. As a general rule, safe mode is used during preliminary MSA operations. In chronological order, MSA HVs are set on LEF MCPs, ST MCPs, TOF chamber, and floating MCP. The TOF chamber and floating MCP voltages are then adjusted before triggering the energy analyzer sweep. During the second MSA commissioning sequence in June 2019, the following results were obtained.

- Nominal voltage $(1500 \mathrm{~V})$ on the LEF MCP stack

- Nominal voltage (2250 V) on the ST MCP stack 
Fig. 53 HEP-ele and HEP-ion with some non-flight items (blue) in a clean bench

- Nominal voltage $(450 \mathrm{~V})$ on the floating MCP

- Half-initial operation voltage $( \pm 4 \mathrm{kV})$ in the TOF chamber

- Fixed voltage $(500 \mathrm{~V})$ in the energy analyzer

To finalize the MSA HV tests, delta commissioning was scheduled in August 2019, but was postponed until early 2020. Delta HV commissioning of MSA was performed at ESOC on February 4 and 5, 2020. All nominal voltages were successfully applied, including TOF chamber VHV ( $\pm 8 \mathrm{kV})$, which will be used in the first phase of MSA operations. The energy analyzer sweep was also successfully triggered, making MSA ready for operations, although $\mathrm{L}$-mode data transmission issue still is under investigation.

\subsection{HEP-ele and HEP-ion}

\subsubsection{Instrument Description of HEP}

The high-energy particle instruments for electrons and ions onboard Mio consist of two sensor heads, HEP-electron (HEP-ele) and HEP-ion. Figure 53 shows photographs of these instruments in a clean bench in the calibration facility at Nagoya University. Non-flight items (blue) protected the flight models during transportation before the final calibrations in April-May, 2014, as will be discussed in Sect. 3.5.3. The specifications of HEP-ele and HEP-ion are summarized in Tables 21 and 22, respectively.

Both instruments are based on the new high-energy particle detection technology developed in Japanese research communities, for the X-ray astrophysics and space physics groups for space missions. The newest space exploration satellite in Japan, the ERG satellite, is also carrying a similar type of high-energy particle detection system using a single-sided strip silicon solid-state detector (SSSD) and an ASIC (VA32TA, IDEAS, Norway: Mitani et al. 2018) as well as the Japanese X-ray Astrophysics mission, Astro-H (Hitomi) (Watanabe et al. 2014). In the HEP instruments onboard Mio, the strip system is not used for position detection, rather, it is used for noise reduction of SSDs owing to the small capacitance and dark current in each strip. In the case of the flight-model HEP-ele onboard Mio, similar to HEP onboard ERG, the SSSD-ASIC system is applied to obtain angular directions of incident electrons used together with a pinslit type aperture. The SSSD-ASIC system reduces the noise level (dark currents) by separating into 158 strip-shaped areas with small capacitance connected and controlled by 5 ASICs. Figure 54 depicts two sections of HEP-ele, in which two separate assemblies of this combination of an SSSD and five ASICs cover up- 
Table 21 Summary of HEP-ele performance

$\begin{array}{ll}\text { Field of view } & \left(18^{\circ} \times 57^{\circ}\right) \times 2 \\ \text { Angular resolution } & 18^{\circ} \times 12^{\circ} \\ \text { Energy range } & 30-700 \mathrm{keV} \\ \text { Energy resolution } & 20 \mathrm{keV}\left(\leq 20^{\circ} \mathrm{C}\right) \\ & \Delta E / E=50 \% \\ \text { Time resolution } & 4 \mathrm{~s}(1 \mathrm{spin})(\text { normal mode }) \\ & 100 \mathrm{~ms}(\text { burst mode }) \\ \text { Geometrical factor } & 0.036 \mathrm{~cm}^{2} \mathrm{sr} \\ \text { Mass } & 0.27 \mathrm{~kg} \\ \text { Power } & 3.04 \mathrm{~W} \\ \text { Dimensions } & 82 \mathrm{~mm} \times 134 \text { mm } \times 115 \mathrm{~mm} \\ \text { Data rate } & 0.0064 \mathrm{kbits} / \mathrm{s}(\mathrm{L}-\text { mode }) \\ & 1.3 \mathrm{kbits} / \mathrm{s}(\mathrm{M}-\text { mode }) \\ & 5.1 \mathrm{kbits} / \mathrm{s}(\mathrm{H} \text {-mode })\end{array}$

Table 22 Summary of HEP-ion performance

\begin{tabular}{ll}
\hline Field of view & $11^{\circ} \times 110^{\circ}$ \\
Angular resolution & $11^{\circ} \times 20^{\circ}$ \\
Energy range & $30-1500 \mathrm{keV}$ \\
Energy resolution & $20 \mathrm{keV}\left(\leq 20^{\circ} \mathrm{C}\right)$ \\
& $\Delta E / E=50 \%$ \\
Time resolution & $4 \mathrm{~s}(1 \mathrm{spin})($ normal mode) \\
& $100 \mathrm{~ms}($ burst mode $)$ \\
Geometrical factor & $0.36 \mathrm{~cm} 2 \mathrm{sr}$ \\
Mass & $1.71 \mathrm{~kg}$ \\
Power & $4.81 \mathrm{~W}$ \\
Dimensions & $212 \mathrm{~mm} \times 169.2 \mathrm{~mm} \times 180 \mathrm{~mm}$ \\
Data rate & $0.0085 \mathrm{kbits} / \mathrm{s}($ energy), $0.0064 \mathrm{kbits} / \mathrm{s}$ (TOF) (L-mode) \\
& $0.77 \mathrm{kbits} / \mathrm{s}($ energy), $0.19 \mathrm{kbits} / \mathrm{s}$ (TOF) (M-mode)
\end{tabular}

and down-looking FOVs from the spacecraft spin plane, respectively. Each SSSD-ASIC assembly has a rectangular SSSD forming a one-layered stack with 158 strips and 5 ASICs to cover $18^{\circ} \times 57^{\circ}$ in total, and an angular resolution of $18^{\circ} \times 11^{\circ}$ is achieved by binning these 158 strips into 5 directions according to their ASIC connections. The accumulated pulse height levels corresponding to the deposited energies in the neighboring three strips are used to calculate the incident particle energy. These slow-shaped pulse height signals from the 10 total ASICs are processed in an analogue-digital converter (ADC) board and an FPGA in the HEP-ele sensor head electronics. Another FPGA is designated for the spacewire interface to the mission data processor (MDP). The analogue-digital conversion of the pulse height for the neighboring three strips is conducted sequentially by the ADC, and their accumulation for the total energy analysis is performed in the FPGA. The incidence direction for each particle detection is also identified and tagged in the FPGA using the ASIC fast-shaped signals. Figure 55 shows the SSSD-ASIC assemblies for HEP-ele in which 158 strips on the SSSDs and 5 ASICs are connected by wire bonding. To avoid contamination by heavier space particles with energies less than several hundreds of kilo electronvolts for 
Fig. 55 SSSD-ASIC assembly of

HEP-ele
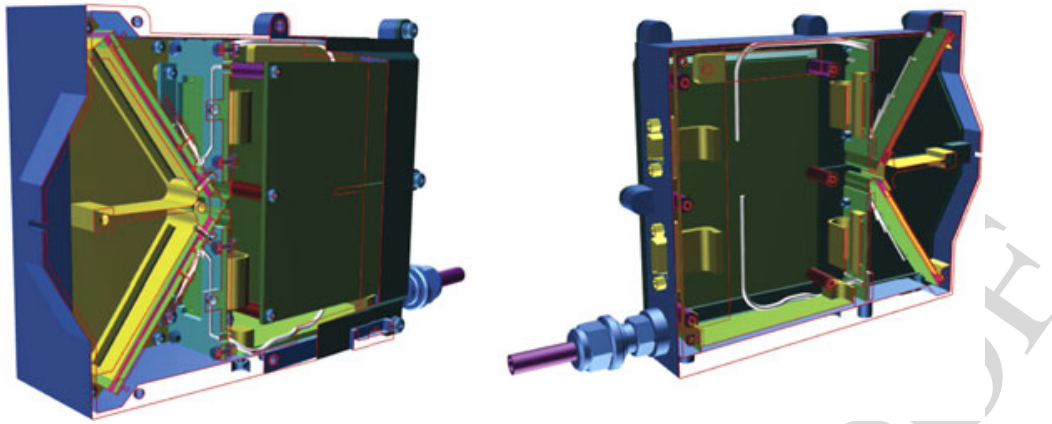

Fig. 54 Cross sections with slightly oblique cutaway of 3D drawing of HEP-ele from two directions

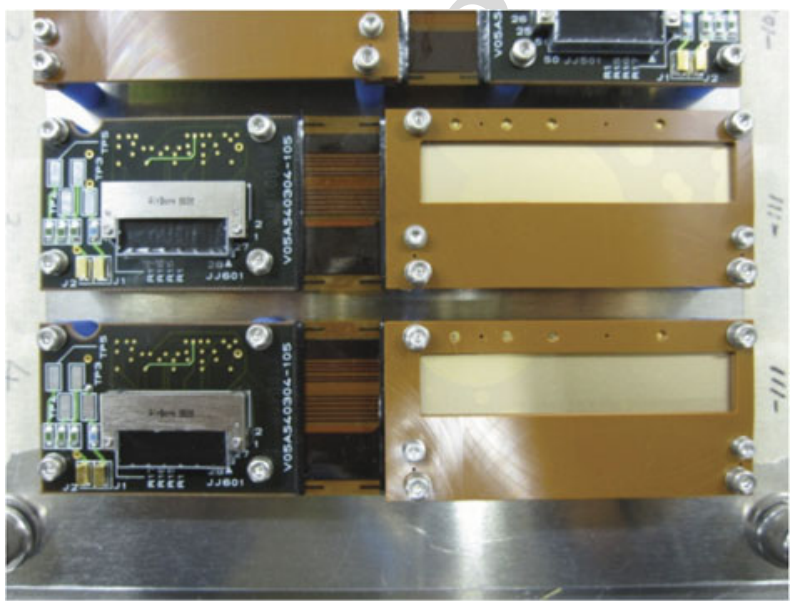

protons and stray solar photons, the incident areas of SSSD are coated by Al with an appropriate thickness of $100 \mu \mathrm{m}$. The inner walls of the detector section are blackened with conductive paint for decreasing the reflected stray photons (Fig. 56). This black carbon painting was developed by Nishiura Paint Industry in Japan (https://www.nishiura-p.com) and was also applied to the plasma particle instruments in the previous Japanese space exploration missions, e.g., PSA-ESA on Nozomi (Planet-B) (Machida et al. 1998) and MAP-PACE on 
Fig. 56 HEP-ele detector section structures blackened with conductive paint

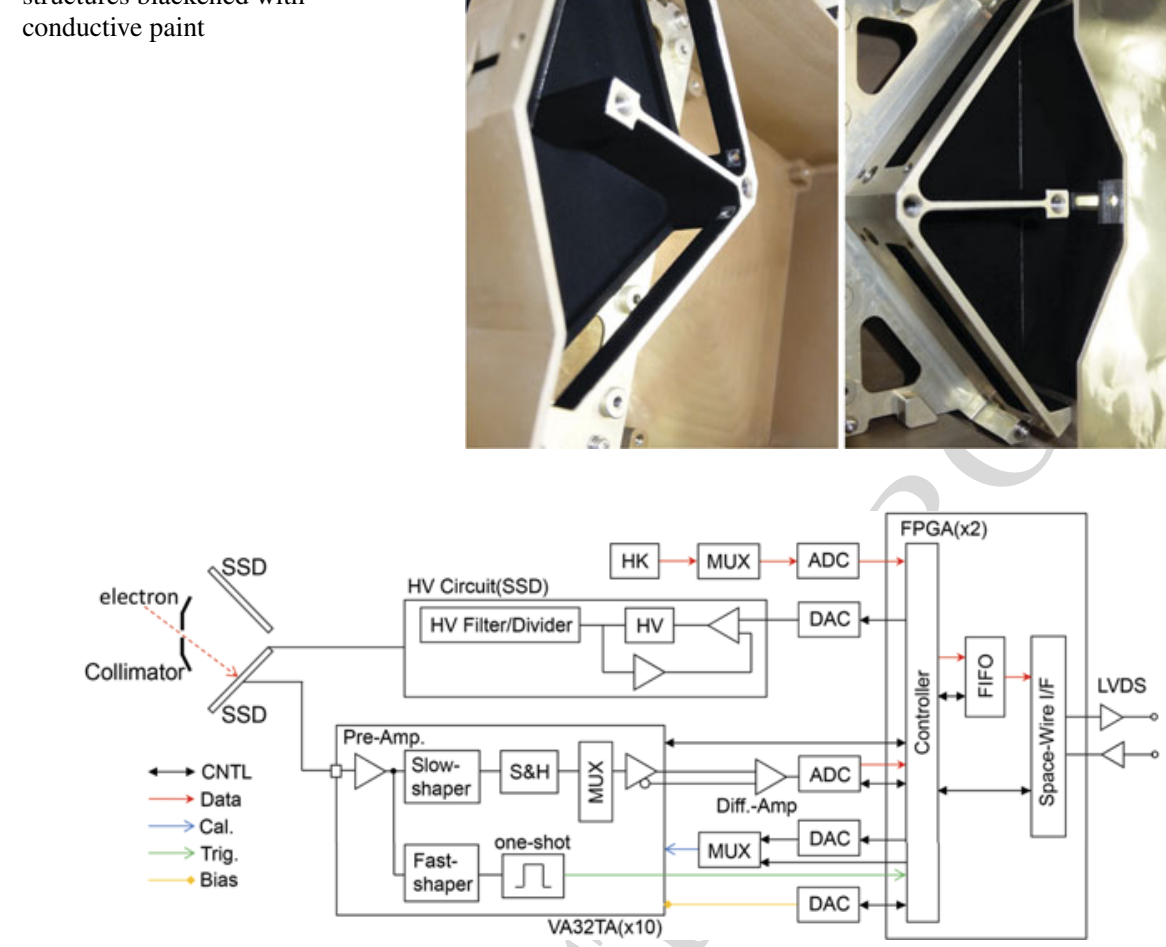

Fig. 57 Block diagram of HEP-ele

Kaguya (SELENE) (Saito et al. 2010b). The entire sensor head of HEP-ele contains highvoltage supply and front-end processing boards behind the detector section that consists of the pinslit aperture and two SSSD-ASIC assemblies. These components and data/signals are controlled/processed by two FPGAs, as schematically illustrated in the block diagram in Fig. 57. The on-board calibration signals and the bias voltages for the pulse discrimination in each ASIC are also issued via DACs from the FPGA. For preventing the intense solar irradiation near the Mercury orbit from entering the detector section through the pinslit aperture, a rectangular thermal shield is equipped in the Mio spin plane in the overall FOV of HEP-ele at the outside of the aperture (Fig. 58). Therefore, the angular area of $\pm 4^{\circ}$ from the spin plane is blocked from the effective HEP-ele FOV by two SSSD-ASIC assemblies as a dead angle range.

The HEP-ion sensor has two measurement capabilities for energy and TOF analyses for the incident ions. These measurement principles, as schematically given in Fig. 59, are essentially the same as those introduced by Saito et al. (2010a). Some structures and performances have been simplified and omitted according to the weight reduction requirement from the viewpoint of the overall spacecraft mission management. The energy/TOF measurement section of the flight-model HEP-ion has five types of components: a conic-shaped collimator, an ultra-thin carbon foil with $0.5 \mu \mathrm{g} / \mathrm{cm}^{2}$ put on electroformed mesh folder with transmission of $66 \%$, two assemblies consisting of three SSSD-ASIC pairs and electron leading meshes, an electrostatic mirror as one of outer structures of the sensor, an MCP assembly with an electron attracting mesh, a TOF start-signal anode and six TOF stop-signal 
Fig. 58 HEP-ele onboard BepiColombo-Mio photographed during the final works before launch

Fig. 59 Measurement principles illustrated on a cross-section of HEP-ion

anodes. The optimal voltages in the TOF analysis unit are also shown in Fig. 59 by red. The SSSD incident surface of the engineering-type model is presented in Fig. 60, in which the electron leading meshes are not set up. As illustrated in Fig. 61, the number of the HEP-ion FOV directions is six, each of which corresponds to one of six SSSD-ASIC pairs for the energy analysis measurement mode or TOF stop-signal anodes of the MCP assembly for the TOF analysis measurement mode. Similar to the data processing in the HEP-ele sensor head, the energy/TOF analysis results are sorted into eight steps. Different from the collimator of HEP-ele, the collimator system of HEP-ion is more complicated because the total FOV configuration of HEP-ion is nearly half of a conical shape rather than a planar type. The closure of the inner conical part of the collimator is designed to protect the ultra-thin carbon foil with a 2-cm diameter from the acoustic vibrations/shocks during the spacecraft launch operation. A biphenyl block sublimable in vacuum is loaded in the cylinder to keep the inner conical collimator closed before the launch operation. Several days after the spacecraft launch, the biphenyl block is sublimated in space to release the inner conical collimator to the measurement position by the extension of a mechanical spring(not shown in Fig. 61). The collimator and the measurement section of HEP-ion are also blackened with the same black paint as that used for HEP-ele (Fig. 62). Because the aperture size of the conical colli- 
Fig. 60 Proto-type SSSD-ASIC assembly for HEP-ion, which is similar to that of the flight model
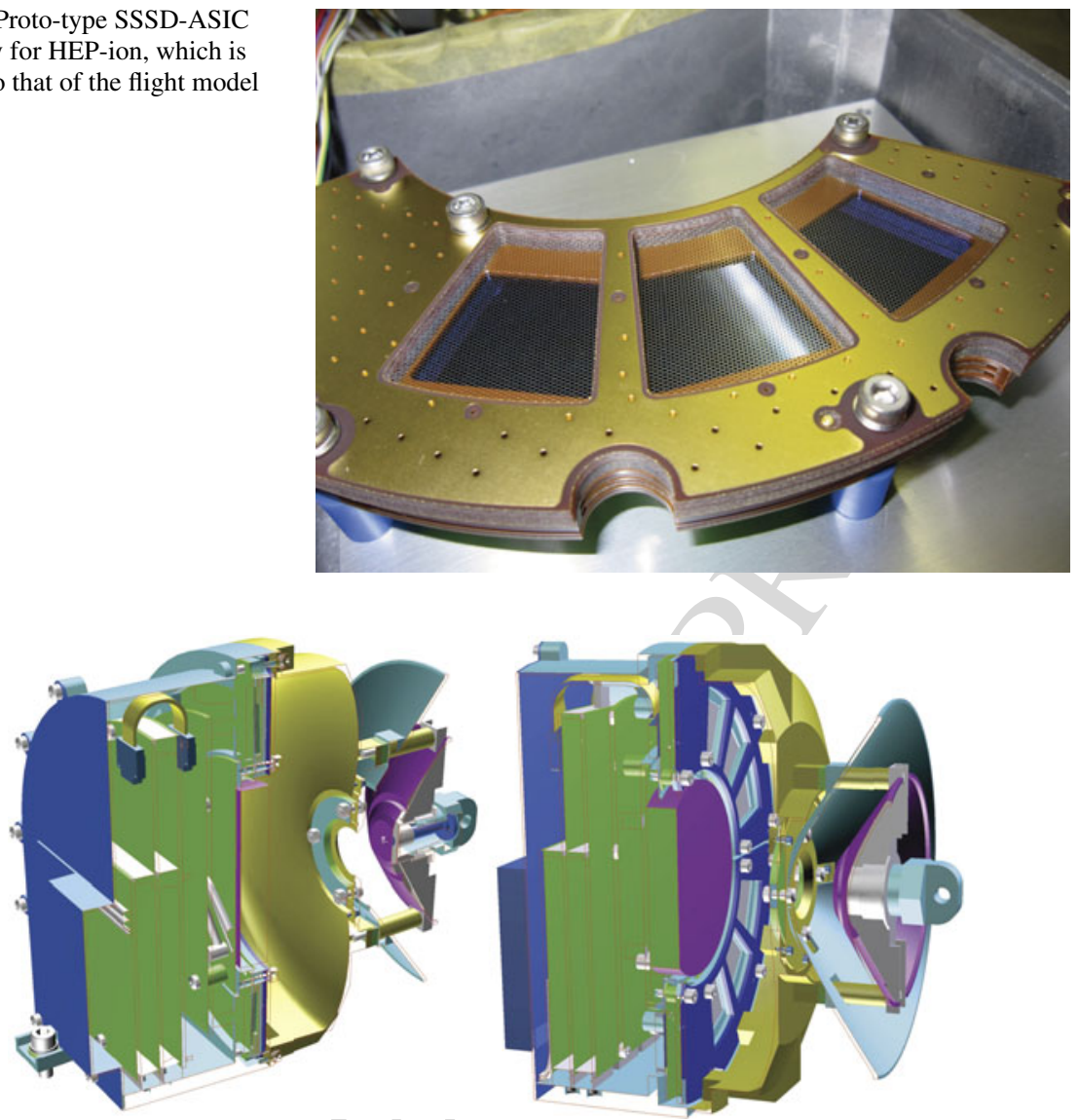

Fig. 61 Cross sections with slightly oblique cutaway of 3D drawings of HEP-ion from two directions

mator of HEP-ion is much larger than that of HEP-ele, as shown in Figs. 54 and 61, the large thermal shield for HEP-ion is installed on Mio (Fig. 63). The dead angle range is $\pm 12.5^{\circ}$ with respect to the spin plane because a side stay blocks the center of the effective HEP-ion FOV between two SSSD-ASIC assemblies. Figure 64 illustrates a block diagram of HEPion, in which the TOF analysis circuit is added to the HEP-ele diagram. It should also be noted that the numbers of the high-voltage supply units (three for HEP-i, one for HEP-e) and the ASICs (six for HEP-i, ten for HEP-e) are different from those of HEP-ele.

\subsubsection{Operation Modes and Data Products of HEP-ele and HEP-ion}

Because of the severe restriction of the telemetry data allocation to HEP-ele and HEP-ion observations, the energy/TOF analysis data need to be compressed by using accumulation over several FOV directions and observational intervals. The measurements themselves are quite simple compared with those of other instruments of MPPE such as MPPE-MSA. As described in Sect. 3.5.1, HEP-ele originally had 10 FOV directions in the spinning spacecraft frame, corresponding to the polar angles in the direction perpendicular to the spin plane, and eight energy steps. However, the spin motion is divided into 16 sectors, which indicates that 
Fig. 63 HEP-ion onboard BepiColombo-Mio photographed during the final works before launch

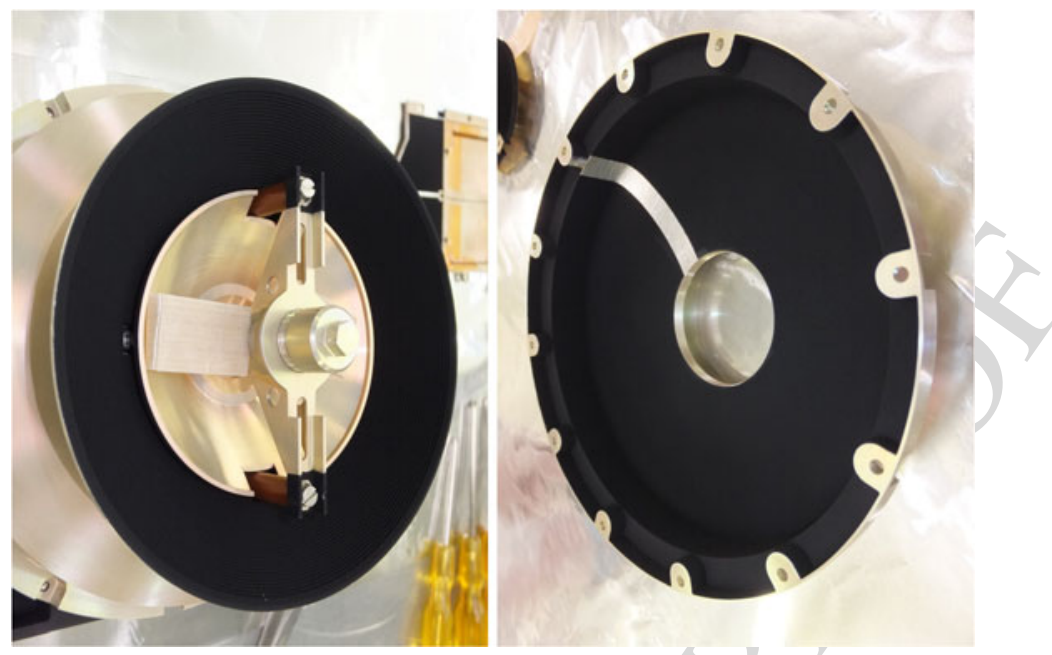

Fig. 62 HEP-ion collimator (left) and electrostatic mirror (right) of the TOF unit blackened with conductive paint

the raw count data in 10 (polar angle) $\times 8$ (energy) $\times 16$ (sector) bins are produced with every spin motion. Whereas HEP-ion has a similar raw count data structure, the number of FOVs is six. The measurement modes of HEP-ion could be switched between the energy and TOF analyses by changing the high voltages applied in the measurement section. These count data could be compressed in MDP1 according to three spacecraft operation modes, e.g., L- and M-modes. The H-mode of HEP-i is not allocated in the current observational plan for reducing the total HEP data because the HEP-e observations and their data with the high data rates are considered to be more important. Tables 23 and 24 show the data modes for HEP-ele and HEP-ion, respectively, in which the values in round brackets indicate the angle resolutions for the polar (FOV) and sector directions. The energy channels for HEP-ele and HEP-ion and the TOF channels of HEP-ion depend on the energy/TOF binning tables selectable by operation commands. 
Table 24 Observation Mode of HEP-ion

Table 23 Observation Mode of HEP-ele

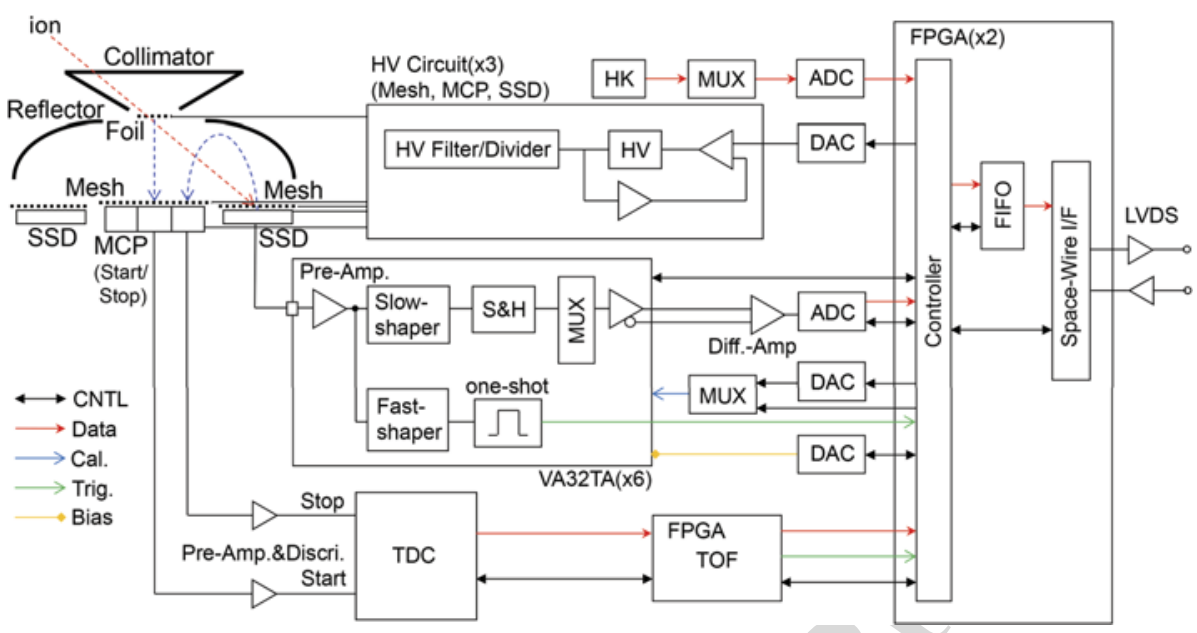

Fig. 64 Block diagram of HEP-ion

\begin{tabular}{lll}
\hline L-mode & Polar FOV $\left(57^{\circ}\right): 2 \times \operatorname{Sector}\left(90^{\circ}\right): 4$ & 48 bytes $/ 15$ spin \\
& $\times$ Energy Step: 3 & 48 bytes $/ 15$ spin \\
M-mode & Polar FOV $\left(11^{\circ}\right): 10 \times \operatorname{Sector}\left(45^{\circ}\right): 8$ & 640 bytes $/ 1$ spin \\
& $\times$ Energy Step:4 & 640 bytes $/ 1$ spin \\
H-mode & Polar FOV $\left(11^{\circ}\right): 10 \times \operatorname{Sector}\left(22.5^{\circ}\right): 16$ & 5120 bytes $/ 1$ spin \\
& $\times$ Energy Step $(95 \mathrm{keV}): 8$ & 5120 bytes $/ 1$ spin \\
\hline
\end{tabular}

Energy analysis mode

\begin{tabular}{lll}
\hline L-mode & Polar FOV $\left(60^{\circ}\right): 2 \times \operatorname{Sector}\left(90^{\circ}\right): 4$ & 64 bytes $/ 15$ spin \\
& $\times$ Energy step $(440 \mathrm{keV}): 4$ & 64 bytes $/ 15$ spin \\
M-mode & Polar FOV $\left(20^{\circ}\right): 6 \times \operatorname{Sector}\left(45^{\circ}\right): 8$ & 384 bytes $/ 1$ spin \\
& $\times$ Energy step $(220 \mathrm{keV}): 8$ & 384 bytes $/ 1$ spin
\end{tabular}

TOF analysis mode

$\begin{array}{lll}\text { L-mode } & \text { Polar FOV }\left(60^{\circ}\right): 2 \times \operatorname{Sector}\left(90^{\circ}\right): 4 & 48 \text { bytes } / 15 \text { spin } \\ & \times \operatorname{TOF}(300 \mathrm{~ns}): 3 \text { bin } & 48 \text { bytes } / 15 \text { spin } \\ \text { M-mode } & \operatorname{Polar} \operatorname{FOV}\left(20^{\circ}\right): 2 \times \operatorname{Sector}\left(45^{\circ}\right): 4 & 96 \text { bytes } / 1 \text { spin } \\ & \times \operatorname{TOF}(150 \mathrm{~ns}): 6 \text { bin } & 96 \text { bytes } / 1 \text { spin }\end{array}$

\subsubsection{Pre-flight Calibration of HEP}

The standalone SSSD-ASIC systems of HEP-ele and HEP-ion were calibrated with a radioactive source $\left({ }^{137} \mathrm{Cs}\right)$ emitting high-energy electrons with energies of more than $600 \mathrm{keV}$ to check the basic performance including the readout capabilities of the ASIC. Figure 65 presents the pulse-height analysis results obtained with 2 of the 158 strips in the SSSDASIC assembly for HEP-ele. Two separate peaks are clear near the uppermost channels 

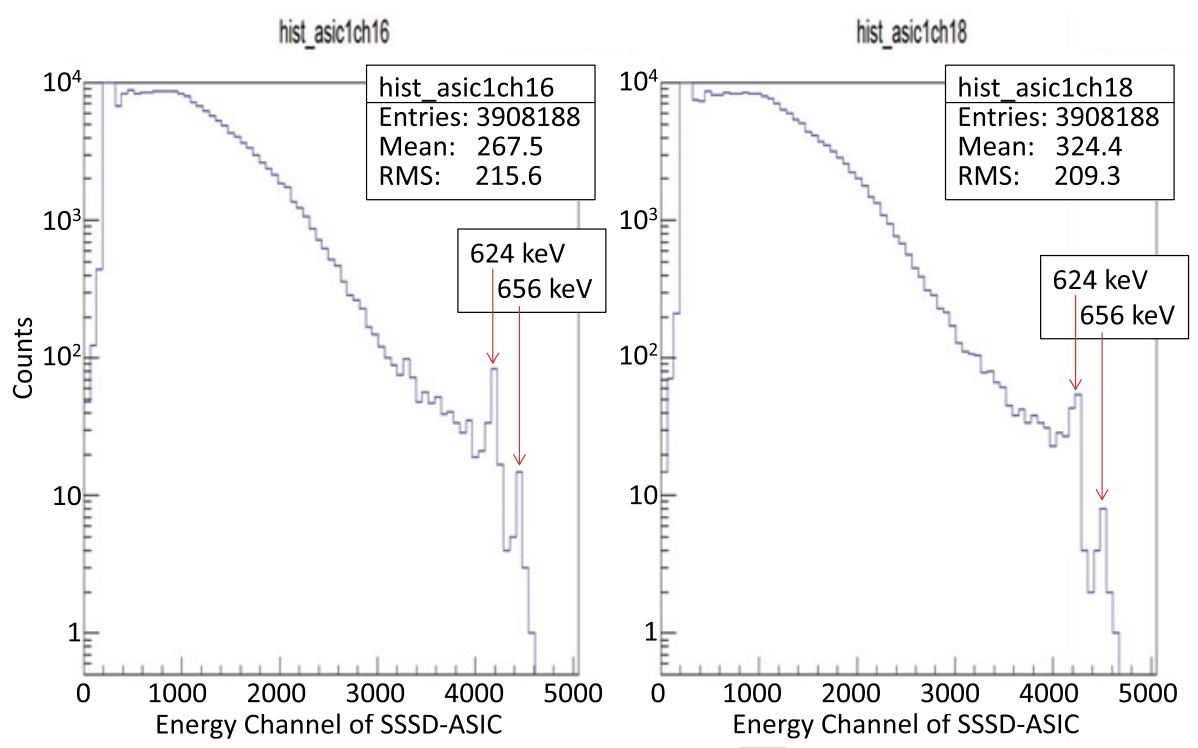

Fig. 65 Examples of pulse height analysis obtained for two strips of the SSSD-ASIC assemblies in HEP-ele

Fig. 66 Calibration facility at the Solar-Terrestrial Environment Laboratory (currently the Institute for Space-Earth Environmental research, ISEE) at Nagoya University

corresponding to electric charges produced in the depletion layer of the SSSD by the incident electrons with $624 \mathrm{keV}$ and $656 \mathrm{keV}$, respectively. Broad distributions spreading over lower channels are caused by contaminations by a continuum component composed of highenergy photons (X-rays and gamma rays) and scattered electrons in a wide range of energy originating from the radioactive source used for the calibration. This type of readout signal from SSSD by ASIC could be analyzed according to the pulse heights for all $158 \times 2=316$ strips in the 2 SSSD-ASIC assemblies and determines the total energy and incident direction for each of the incident electrons.

The electron/ion beamlines in the calibration facility at Nagoya University (Fig. 66) were also used to check the performance in the lower-energy ranges of the HEP-ele and HEP-ion measurements. The energy ranges used for the HEP instruments were $40-100 \mathrm{keV}$ for elec- 
Fig. 67 HEP-ion set on the multi-axial turntable system in the vacuum chamber at the beamline calibration facility

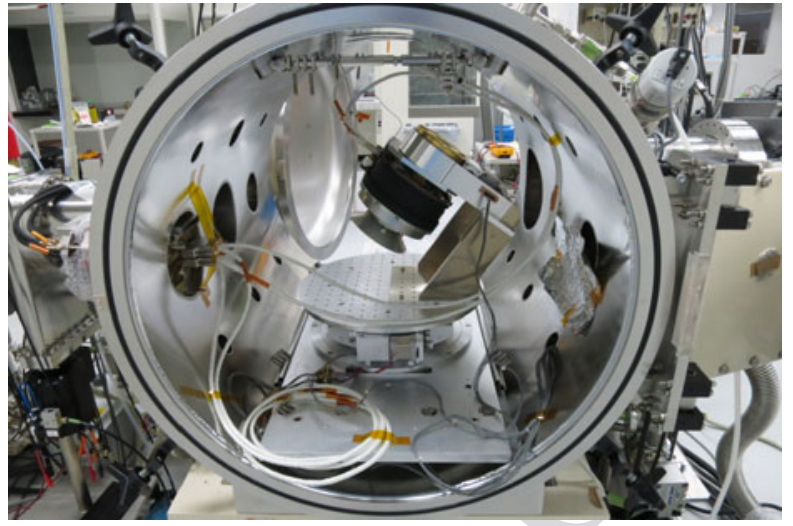

trons and 60-140 keV for protons, and ions of $\mathrm{He}$ and N. To check the instrumental response according to the incident angles, we rotated the HEP-ele or HEP-ion instrument around three independent axes in the vacuum chamber during the beamline calibrations. Using a multiaxial turntable system, the FOV direction was set parallel to or oblique by aimed angle with respect to the charged particle beam with diameters of a few tens of millimeters at a given energy. Figure 67 shows the HEP-ion instrument with the harness set on the turntable system in the vacuum chamber.

The histograms showing the pulse height distributions produced by irradiating electrons with the energy of $100 \mathrm{keV}$ are given in Fig. 68, in which six plots are correspondent to incident (polar) angles identified by the strip and ASIC numbers of HEP-ele. In each plot, two distinct peaks present a so-called pedestal (background noise level) owing to the SSSDASIC characteristics and an actual signal distribution for the $100-\mathrm{keV}$ electron energy deposits in the depletion layer of the SSSD (e.g., Mitani et al. 2018; Kasahara et al. 2009). The channel numbers of the peak distributions in the abscissa are not always identical for all six plots because the dark current levels measured in the 10 ASICs could be different. These level differences among the 10 ASICs are subtracted after the pulse height analyses in the HEP-ele FPGA procedures to achieve sufficient energy discrimination capability over the entire energy range of HEP-ele. Similar calibrations have been performed for two SSSD-ASIC assemblies in HEP-ion by emitting typical types of ion species with several levels of energies. Figure 69 shows examples of the pulse height analyses for $140-\mathrm{keV}$ protons, in which six panels correspond to six FOV directions, each of which is detected by the corresponding ASIC. The lower portions of the energy distributions for the $140-\mathrm{keV}$ proton beams overlap with the pedestal distributions at lower channels than the proton distributions, which is different from the distributions in Fig. 68. This occurred because the energies of ions injected into the solid state detectors with a certain thickness of the dead layer can be reduced more significantly than those of electron cases. Similar to the HEPele energy distributions in Fig. 68, the energy and pedestal peaks are not identical for six ASICS of HEP-ion regarding the energy channels in the abscissas. These channel discrepancies among the ASICs can be reduced by the onboard routine process for channel difference subtraction in the FPGA of HEP-ion, which is also similar to the HEP-ele procedures.

The HEP-ion instrument has the capability to measure the velocities of the injected particles by using the TOF unit, as described in the previous subsection. We also checked the TOF performance with the ion beamlines at Nagoya University and JAXA. Figure 70 summarizes the TOF experimental results for four different energies $(60,120,250,1000 \mathrm{keV})$ and three ion species (proton, singly-charged $\mathrm{He}$ and $\mathrm{N}$ ). Because the heavy ion beams were 

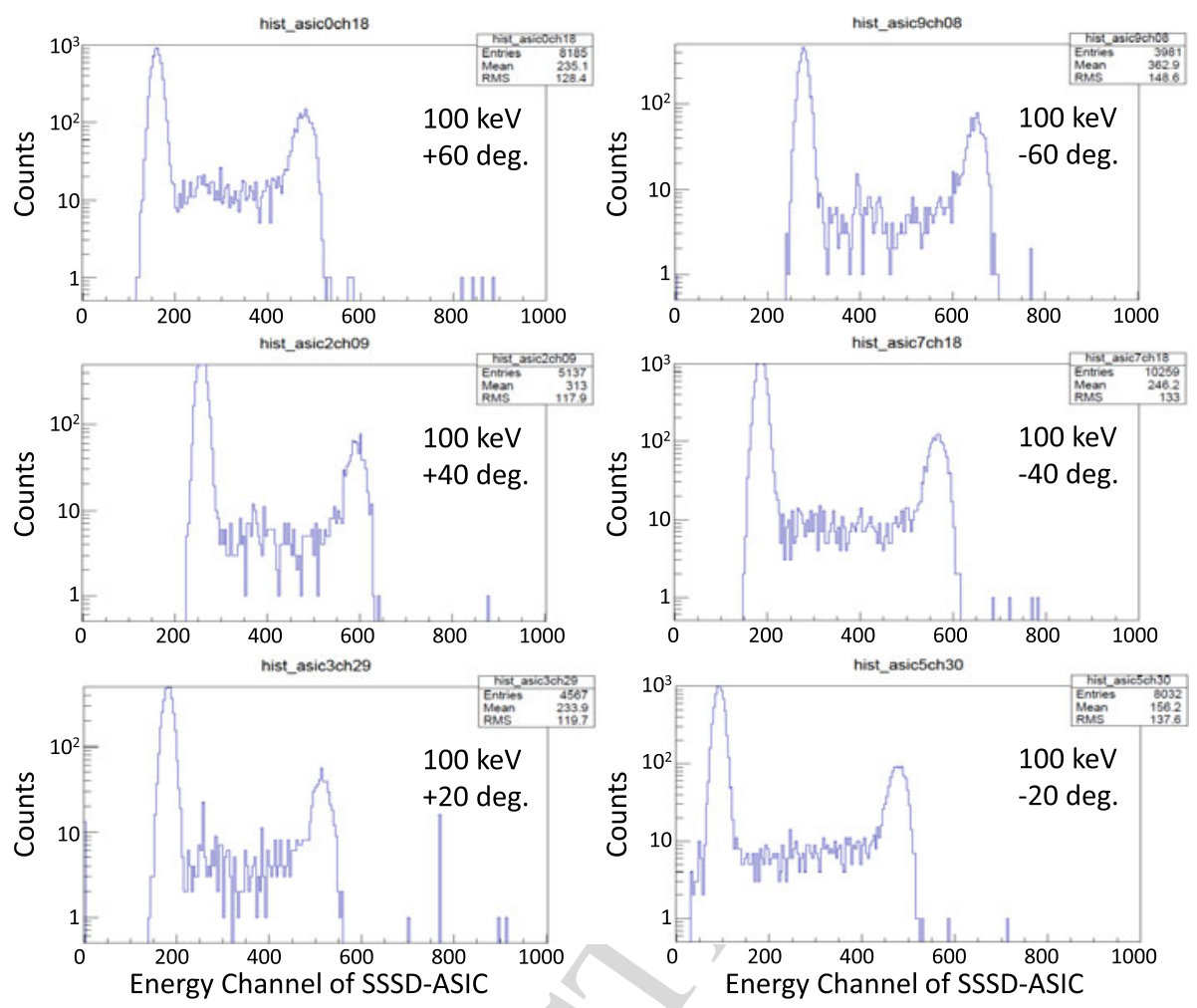

Fig. 68 Examples of pulse height analysis for six incident (polar) angles obtained in the correspondent strips of two SSSD-ASIC assemblies in HEP-ele

not applicable beyond $150 \mathrm{keV}$ owing to the JAXA high-energy beamline facility performance, the TOF distributions were measured only for $\mathrm{H}^{+}$. The incident FOV directions were changed in these measurements so that the maximum peaks were obtained in the different ASIC assemblies as indicated by different colors in the histograms.

\subsubsection{Near-Earth Commissioning Results of HEP}

The first in-flight operations for HEP-ele and HEP-ion were conducted during the near-Earth commissioning phase of the BepiColombo mission. High-voltage up to $98.2 \mathrm{~V}$ was supplied to two SSSD assemblies of HEP-ele. We confirmed the normal HK status including the instrument currents and significantly low dark counts. The six HEP-ion SSSDs were also checked to be activated by the operation with the high-voltage up to $99.7 \mathrm{~V}$, in which the instrument currents and HK status were confirmed to be normal. The activation of the TOF unit of HEP-ion was performed safely to obtain the normal HK status with the high-voltages supplied up to $990 \mathrm{~V}$ and $2271 \mathrm{~V}$ to the mesh system deriving the start/stop electrons and the MCP assemblies, respectively. The dark counts were measured steadily as constant MCP noise. 

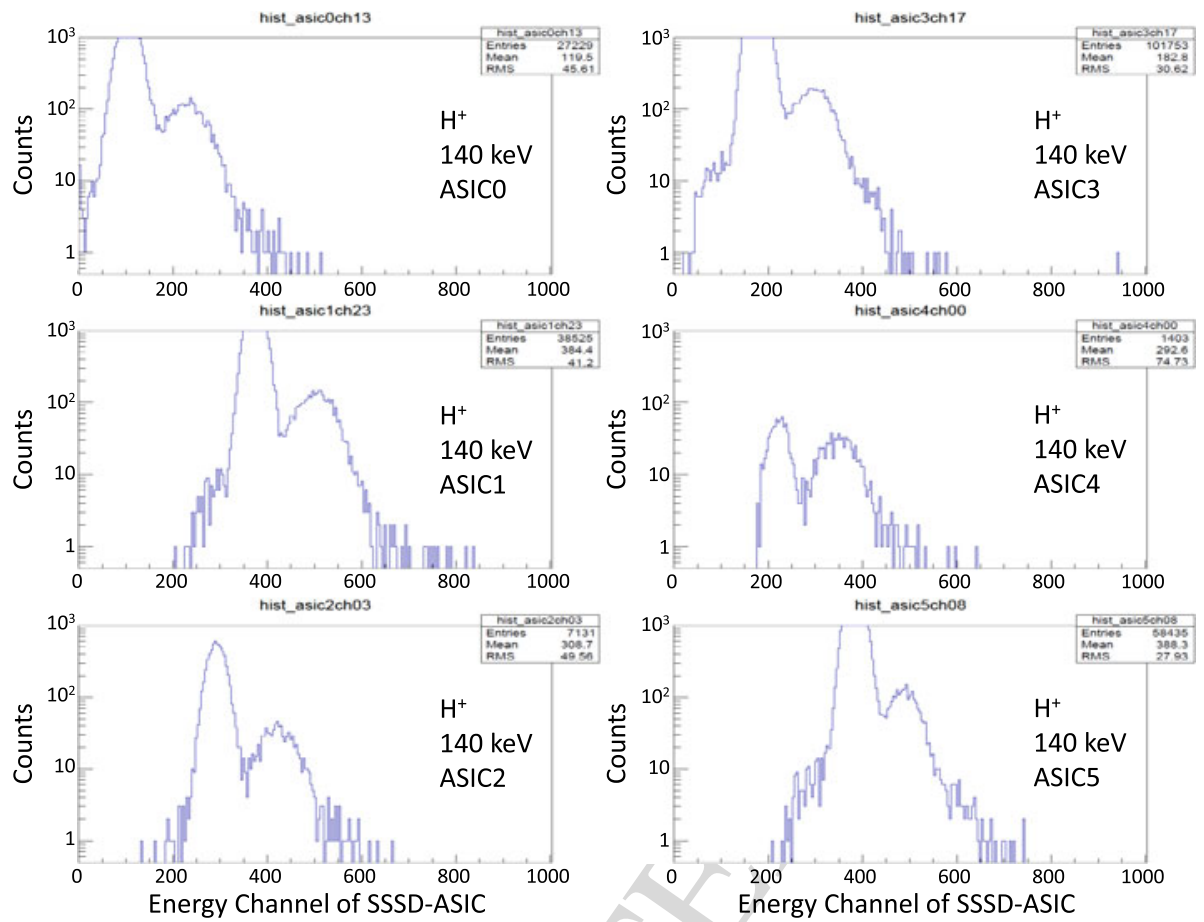

Fig. 69 Examples of pulse height analysis for six incident (polar) angles corresponding to the SSSD-ASIC pairs in HEP-ion

\subsection{ENA}

\subsubsection{Instrument Description of ENA}

The ENA instrument is based on the surface conversion/reflection technique and consists of four subsystems, including an ion rejection system, ionization surface, photon rejection system that also performs crude energy analysis, and velocity analysis section (Kazama et al. 2009). Figures 71, 72, 73, and 74 show the concept, schematic view, flight model of the instrument, and sun shield mounted on the spacecraft, respectively.

Neutrals enter the sensor through an electrostatic charged particle deflector, which rejects ambient charged particles by a static electric field. The incoming neutrals are then converted to positive ions on an ionization surface and then pass through an electrostatic analyzer of a specific (wave) shape that effectively blocks photons. The electrostatic analyzer also provides crude energy analysis. The "wave" electrostatic analysis design is similar to that used in the MTOF sensor of the CELIAS instrument on the SOHO spacecraft (Hovestadt et al. 1995), which provides a photon rejection factor of $2 \times 10^{-8}$. Because the instrument must be capable of measuring masses up to $\mathrm{Fe}$, no foils can be used in the following TOF section. To measure the particle velocity (mass), we used the particle reflection principle developed for and utilized in the Neutral Particle Detector (NPD) of the ASPERA-3 and -4 experiments (Barabash et al. 2006, 2007) for ESA's Mars and Venus express missions. After exiting the electrostatic analyzer, ions originally neutrals that were converted to ions by the ionization surface are post accelerated up to an energy of $1.5 \mathrm{keV}$ and impact the START 
3201

3202

3203
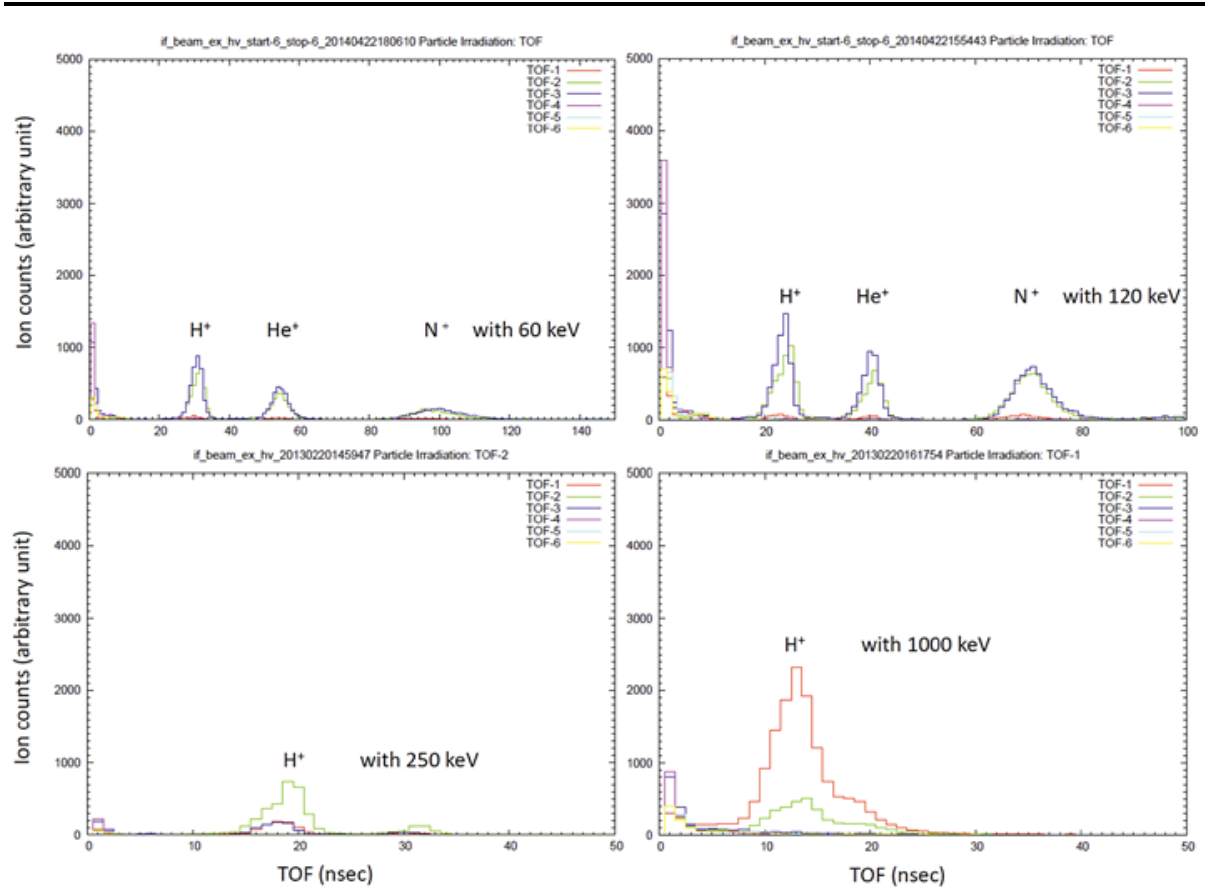

Fig. 70 TOF profiles for ions with energies of 60, 120, 250, and $1000 \mathrm{keV}$. Only protons were available for energies beyond $150 \mathrm{keV}$ because of the beamline facility performance

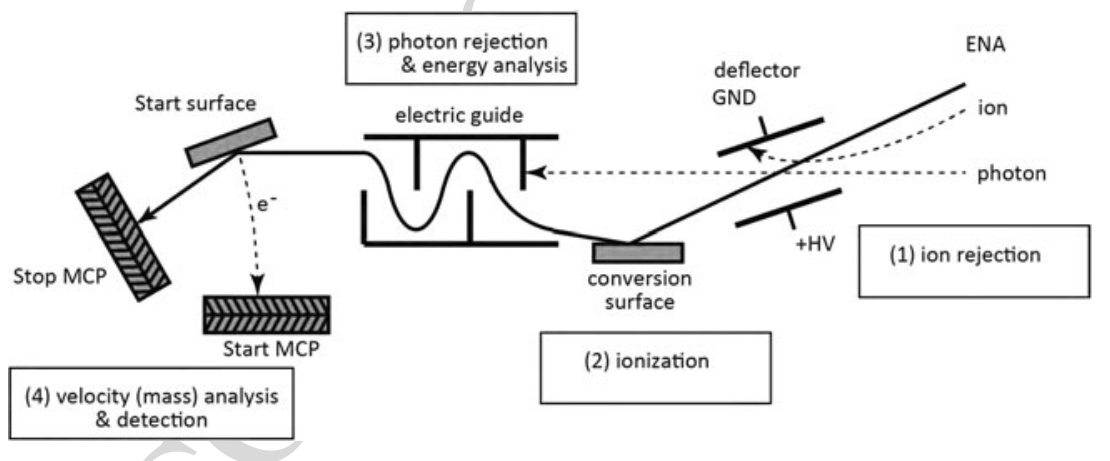

Fig. 71 ENA measurement concept

surface under a grazing angle of $15^{\circ}$. During the impact, kinetic secondary electrons are emitted and the particles are reflected toward the STOP MCPs, where they are detected and produce a STOP pulse. The secondary electrons from the START surfaces are guided to the START MCPs and produce a START pulse. The START and STOP timing gives the particle velocity. Combining the TOF measurements and electrostatic analyzer settings one determines the LENA energy and mass. Measuring the radius and azimuth of the neutral hit on the START surface by position sensitive START MCPs enables accurate determination of the TOF length and the arrival azimuth of the incoming neutrals. Figure 75 shows the ENA 
3251

3252

3253

3254

3255

3256

3257

3258

3259

3260

3261

3262

3263

3264

3265

3266

3267

3268

3269

3270

3271

3272

3273

3274

3275

3276

3277

3278

3279

3280

3281

3282

3283

3284

3285

3286

3287

3288

3289

3290

3291

3292

3293

3294

3295

3296

3297

3298

3299

3300

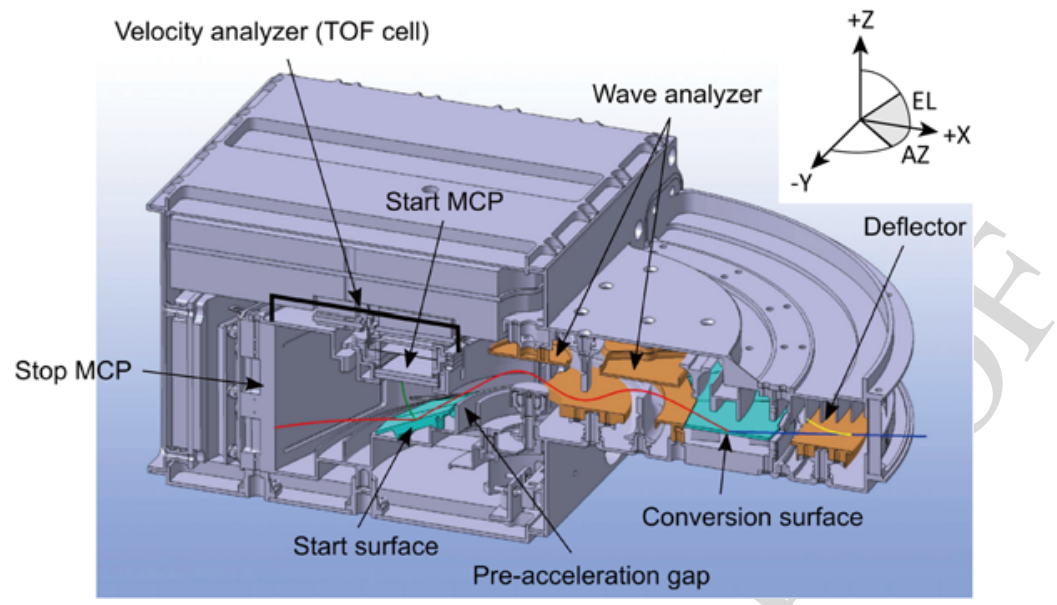

Fig. 72 Sectional view of the ENA sensor

Fig. 73 ENA flight model. The sensor is wrapped by MLI, when ENA is installed in the spacecraft

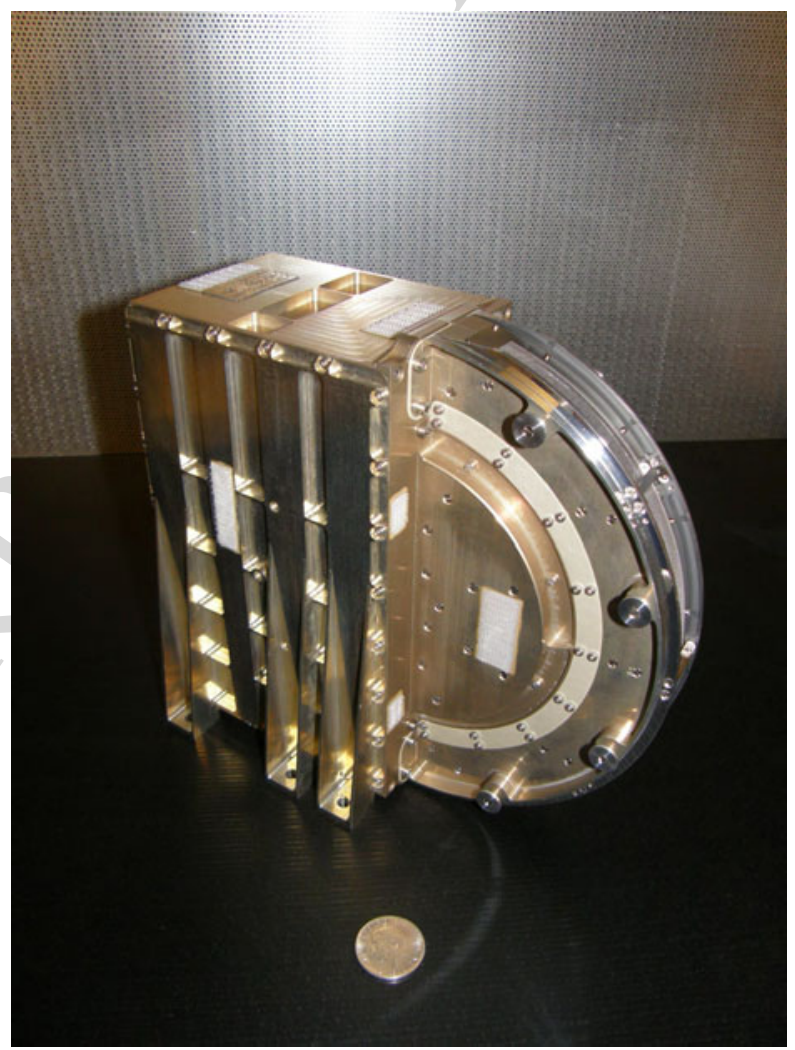

block-diagram. The instrument electronics include two boards, interface electronics (IFE) and HVPS. The DC/DC converters and digital processing are provided externally. The ENA sensor characteristics are summarized in Table 25.

Springer

Journal ID: 11214, Article ID: 839, Date: 2021-07-22, Proof No: 2, UNCORRECTED PROOF 
Fig. 74 ENA sun shield

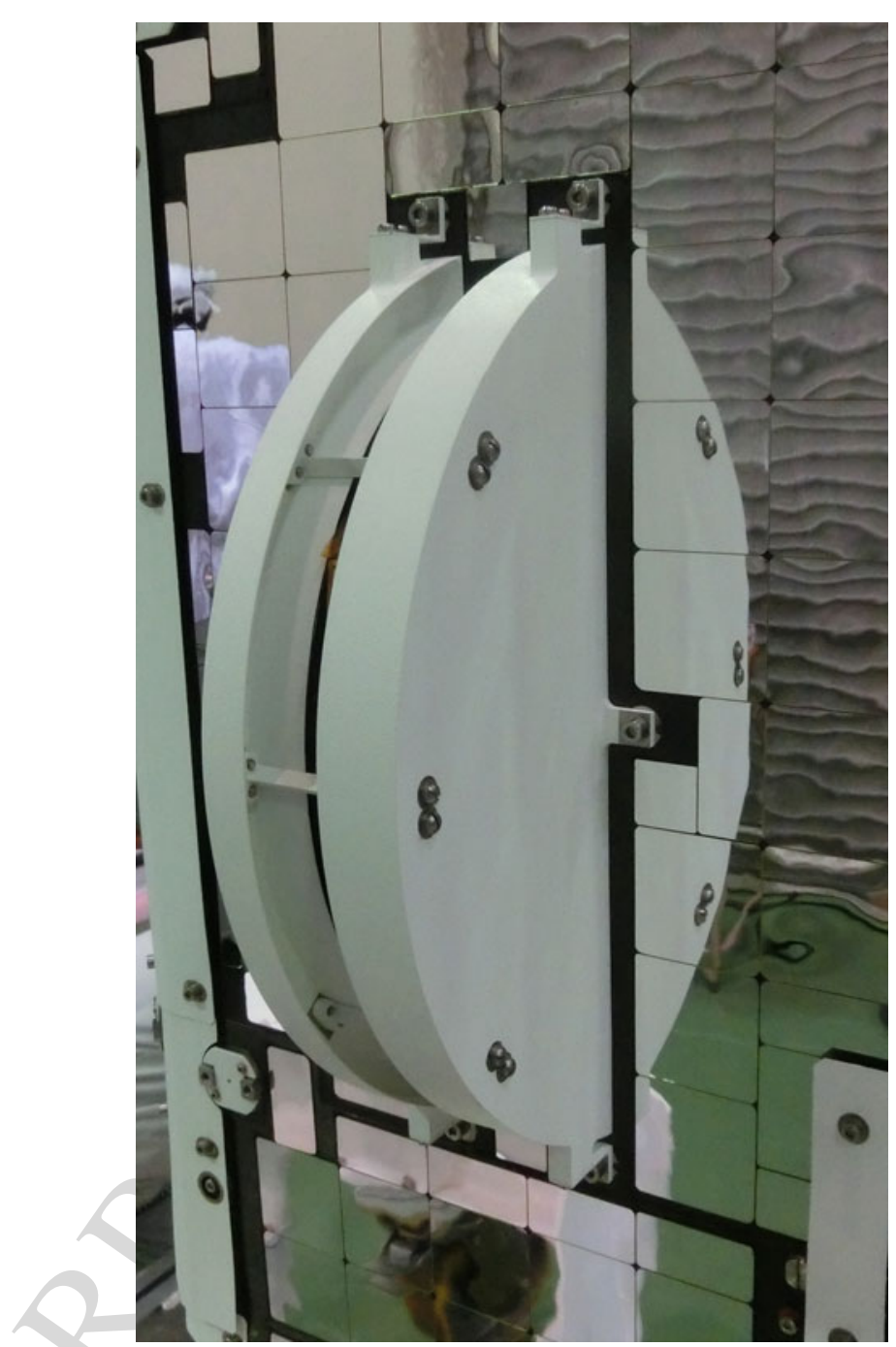

The ENA sensor comprises six key elements: (1) conversion surface, (2) photon absorbing surfaces, (3) START and STOP MCP assemblies, (4) START surface, (5) IFE, which provides front-end-electronics functions, sensor control, and interface with the MPPE DPU (MDP1), and (6) HVPS.

\section{(1) Conversion surface}

After passing the electrostatic analyzer, LENAs hit a conversion surface under a grazing angle of $15^{\circ}$, where they are converted to positive ions. The surface is $\mathrm{Al}_{2} \mathrm{O}_{3}$ (alumina) deposited on a polished (highly smooth) substrate, such as a silicon wafer. A photograph of the conversion surface is shown in Fig. 76. The temperature of the conversion surface is controlled to stay above $+50{ }^{\circ} \mathrm{C}$ throughout the mission to avoid a stack of contaminating materials that would decrease the efficiency of neutral to ion conversion. The conversion surface element has two heater systems. One is operated by the heater control system on- 
Table 25 Summary of ENA performances

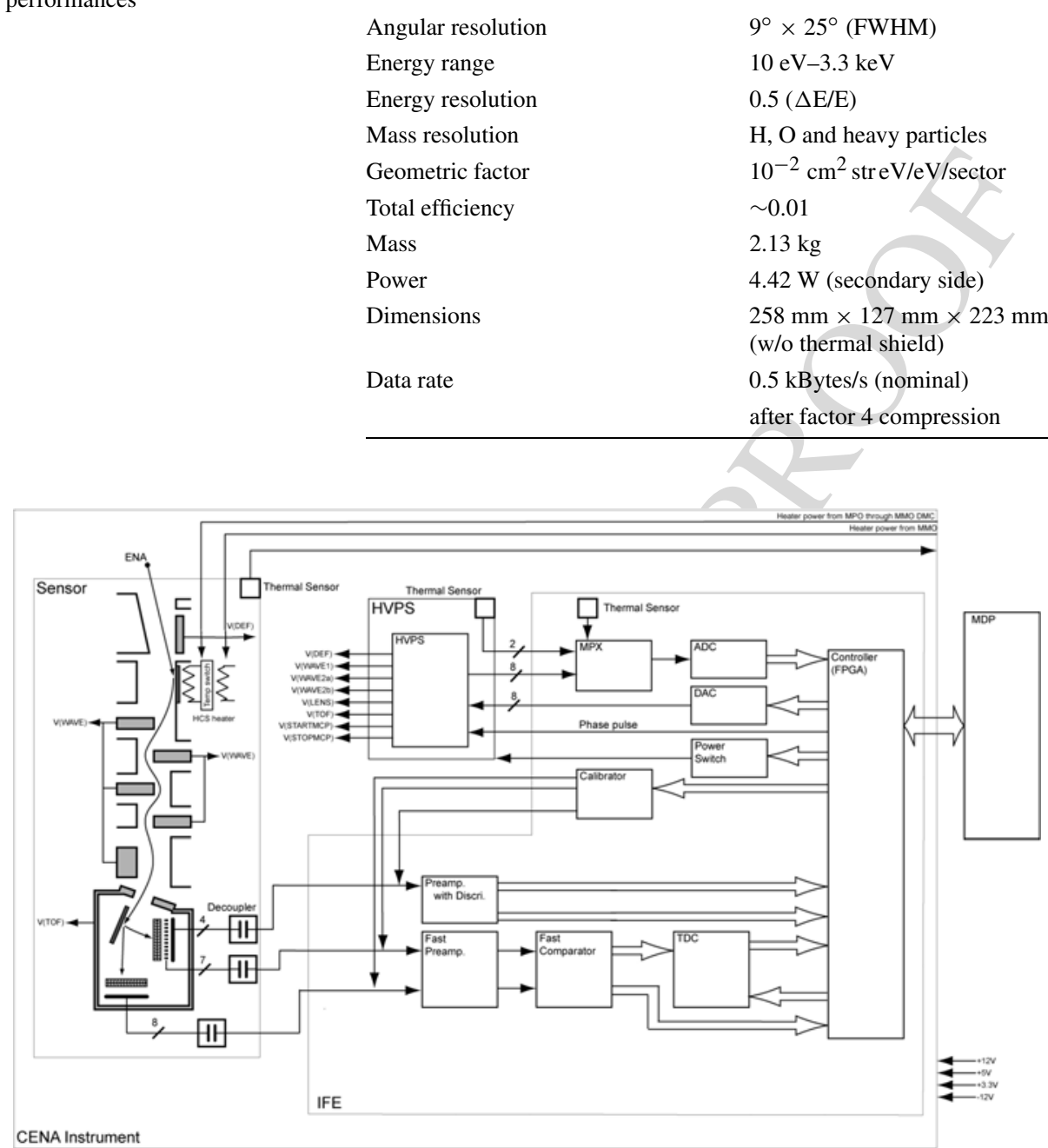

\begin{tabular}{ll}
\hline Field of view & $15^{\circ} \times 160^{\circ}$ \\
Angular resolution & $9^{\circ} \times 25^{\circ}(\mathrm{FWHM})$ \\
Energy range & $10 \mathrm{eV}-3.3 \mathrm{keV}$ \\
Energy resolution & $0.5(\Delta \mathrm{E} / \mathrm{E})$ \\
Mass resolution & $\mathrm{H}, \mathrm{O}$ and heavy particles \\
Geometric factor & $10^{-2} \mathrm{~cm}^{2}$ str eV/eV/sector \\
Total efficiency & $\sim 0.01$ \\
Mass & $2.13 \mathrm{~kg}$ \\
Power & $4.42 \mathrm{~W}(\mathrm{secondary} \mathrm{side)}$ \\
Dimensions & $258 \mathrm{~mm} \times 127$ mm $\times 223 \mathrm{~mm}$ \\
& $(\mathrm{w} / \mathrm{o}$ thermal shield $)$ \\
Data rate & $0.5 \mathrm{kBytes} / \mathrm{s}$ (nominal) \\
& after factor 4 compression \\
\hline
\end{tabular}

Fig. 75 ENA block diagram

board MMO. The other is powered by MPO and is controlled by the ENA heater control and temperature monitoring system (ENA HCS) installed in the ENA instrument. This is necessary because MMO is turned off most of the time during the cruising phase to Mercury. The power for ENA HCS is separated from the other ENA electronics.

\section{(2) Photon absorbing surfaces}

The large electrodes in the wave electrostatic analyzer are specially designed coated plates with high photon absorbing surfaces. The grooves on the plates follow the design for the PLASTIC instrument on SOHO (Galvin et al. 2008). The coating is CuS. This structure serves as a very efficient trap for photons. The total UV transmittance of the system before the TOF section is $<10^{-9}$. 
Fig. 76 Conversion surfaces of ENA

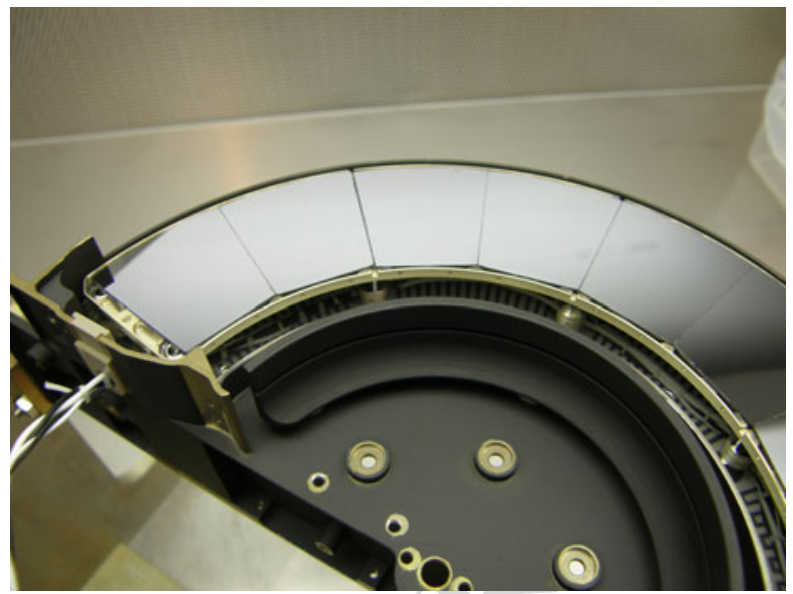

\section{(3) START and STOP MCP assemblies}

The START MCPs are used for both START timing and the determination of the START hit position (radius and azimuth). A chevron of annular MCP plates with an outer diameter $100 \mathrm{~mm}$ is used. The electron cloud exiting the back of the MCP is split between a grid and a plate discrete anode. The grid is divided into seven decoupled sectors to give seven azimuths and seven START timings. The plate anode is divided into four concentric rings to provide determination of the TOF length, where the TOF path length range is from $34 \mathrm{~mm}$ to $60 \mathrm{~mm}$. For each individual case, uncertainty of about $\pm 5 \mathrm{~mm}$ is present owing to the different orientations of the START surface and the STOP MCP front planes. The STOP MCPs are used for STOP timing and determination of the STOP hit position (only azimuth), with four identical assemblies used. The anode behind each MCP assembly is divided into two parts, giving eight independent outputs. Figure 77 shows the channel definition of the MCP anodes.

\section{(4) START surface}

The START surface provides effective reflection of the particles (high reflection coefficient, narrow scattering angle) and high secondary electron yield. The secondary electron yield can be optimized by increasing the post acceleration voltage, and the reflection coefficient can be increased by choosing materials for the START surface with high atomic numbers. The START surface is mono-crystalline tungsten. Figure 78 shows a photograph of the START surface installed in the electrode structure of the TOF section.

\section{(5) Interface electronics}

The IFE has the following functions:

- Amplification of MCP output signals

- Measurement of the time between START and STOP signals

- Generation of calibration pulse

- HVPS control and monitoring its output levels

- Communication with MDP1 (reception of commands and transmission of measured data)

IFE has 19 charge sensitive preamplifiers - shapers including seven fast preamplifiers for seven START sectors, eight fast preamplifiers for eight STOP plates and four slow preamplifiers for four START rings. One TOF unit accepts START signals from any of the seven 
Fig. 77 Channel definition of the $\mathrm{MCP}$ anodes of ENA. The lower panel showing the channel definition of the STOP MCP definition of the START MCP anode which is the same as that in the upper panel anodes includes the channel

\section{MMO MPPE ENA \\ START MCP Anode Channel Definition (View from START MCP input surface)}

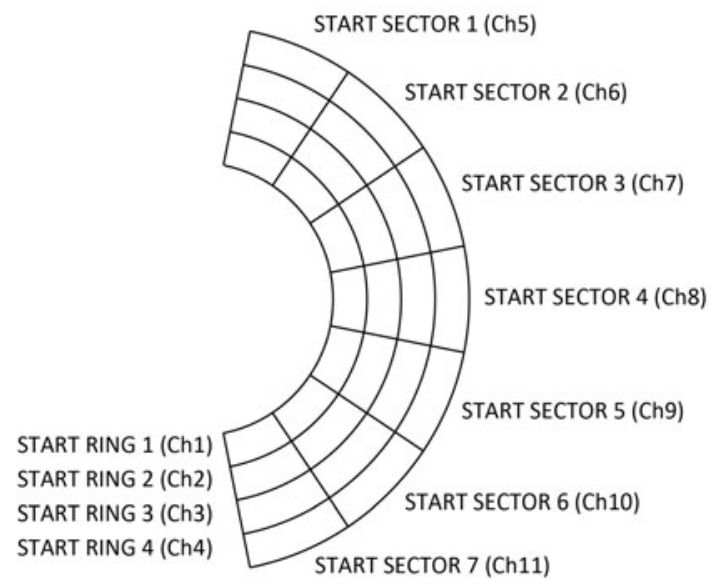

STOP MCP Anode Channel Definition

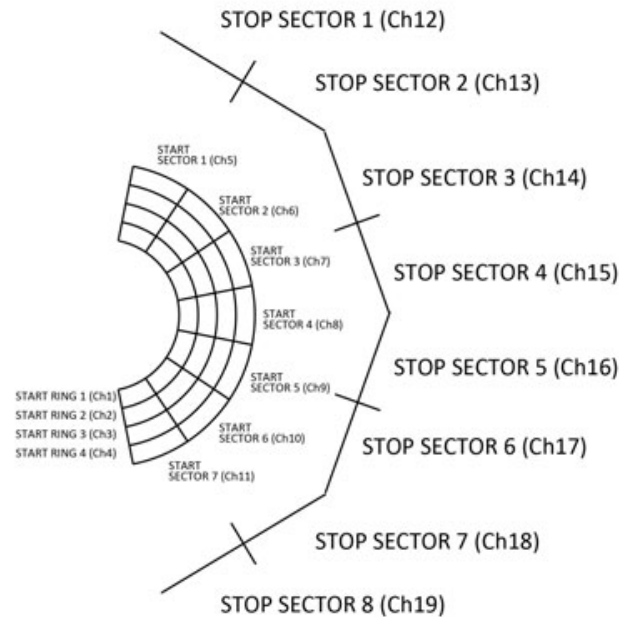

START sectors and STOP signals from any of the eight STOP plates. IFE also contains the necessary ADC and DAC (analog-to-digital and digital-to-analog converters) needed for HVPS control.

\section{(6) HVPS}

One double (positive/negative) supply provides the high voltages applied to the sensor electrodes and bias voltages for MCPs, which is then regulated by optocouplers to the nominal values. Figure 79 shows a photograph of the assembled configuration of HVPS, the STOP MCP assembly, and the preamplifier/digital processing electronics boards. 
Fig. 79 Assembled configuration of the HVPS, STOP MCP assembly, and preamplifier/digital processing electronics boards

Fig. 78 START surface installed in the electrode structure of the TOF section

\subsubsection{Operation Mode and Data Products of ENA}

\section{Operation mode of the ENA sensor}

The ENA sensor has mostly full solid angle coverage (12.0 sr FOV) for ENA detection by using spacecraft spin motion. To obtain angular resolution for the spinning direction, the spin period (Ts: nominally $4 \mathrm{~s}$ ) is divided into 16 spin sectors, where the energy scan of 8 steps is performed for each spin sector. ENA sends a data packet with fixed size $(3,072$ byte $=384$ byte $\times 8$ energy steps) to MDP1 every spin sector.

The ENA sensor has four operation modes: (1) coincidence mode, (2) counter mode, (3) engineering mode, and (4) table read mode. The coincidence mode provides the count and TOF values shown in Table 26. When the ENA sensor detects ENA signals, it gets four types of information such as ID of sectors (START SECTOR, START RING, and STOP SECTOR), which detect the signals, and TOF value for the time interval between the signal detection at START and STOP sectors. The ENA sensor generates raw data that contain this information in 20 bits (TOF event data, Table 27) for each event of particle detection. However, if the occurrence rate of the particle detection events is too high, the sensor cannot 
Table 26 Format of coincidence mode packet of ENA

\begin{tabular}{|c|c|c|}
\hline \multicolumn{2}{|l|}{$\mathrm{TI}$} & 48 bit \\
\hline & & 8 bit $(0 x 00)$ \\
\hline slotID & & 8 bit \\
\hline $\begin{array}{l}\text { TOTAL } \\
\text { COINCIDENCE } \\
\text { COUNT }\end{array}$ & $\begin{array}{l}\text { Incremented when signals from START SECTOR, START } \\
\text { RING, and STOP SECTOR are detected and TOF is } \\
\text { calculated. }\end{array}$ & 16 bit \\
\hline $\begin{array}{l}\text { TOTAL START } \\
\text { COUNT }\end{array}$ & & 16 bit \\
\hline $\begin{array}{l}\text { TOTAL STOP } \\
\text { COUNT }\end{array}$ & & 16 bit \\
\hline $\begin{array}{l}\text { COINCIDENCE } \\
\text { START SECTOR } \\
\text { COUNT }\end{array}$ & $\begin{array}{l}\text { Incremented when signals from START SECTOR, START } \\
\text { RING, and STOP SECTOR are detected and TOF is } \\
\text { calculated. }\end{array}$ & $112 \mathrm{bit}=7 \mathrm{ch} \times 16 \mathrm{bit}$ \\
\hline $\begin{array}{l}\text { COINCIDENCE } \\
\text { STOP SECTOR } \\
\text { COUNT }\end{array}$ & $\begin{array}{l}\text { Incremented when signals from START SECTOR, START } \\
\text { RING, and STOP SECTOR are detected and TOF is } \\
\text { calculated. }\end{array}$ & $128 \mathrm{bit}=8 \mathrm{ch} \times 16 \mathrm{bit}$ \\
\hline TOF event data & & $\mathrm{N} \times 20$ bit \\
\hline Remainder & & Filled with zero \\
\hline $\begin{array}{l}\text { Total } \\
(=(1 / 128) * \text { Ts })\end{array}$ & & 3072 bit \\
\hline
\end{tabular}

\section{TI: Time indicator} slotID: Incremented every energy step $(=(1 / 128) *$ Ts $)$. Cleared by spin pulse

$* 1$ This table shows a format for 1 energy step

*2 MDP1 will read the data every 8 energy steps

Table 27 TOF event data

* $\mathrm{TOF}=0 \mathrm{x} 000$ is dealt with no data marker by MDP1

If it appears, MDP1 will not process the data appeared afterword in the packet

\begin{tabular}{|c|c|c|}
\hline $\begin{array}{l}\text { START } \\
\text { RING }\end{array}$ & 3 bit & $\begin{array}{l}0: \text { ring } 1,1: \operatorname{ring} 2,2: \operatorname{ring} 3,3: \operatorname{ring} 4 \text {, } \\
7: \text { none }\end{array}$ \\
\hline $\begin{array}{l}\text { START } \\
\text { SECTOR }\end{array}$ & 3 bit & $\begin{array}{l}0: \operatorname{sect} 1,1: \operatorname{sect} 2,2: \operatorname{sect} 3,3: \operatorname{sect} 4, \\
4: \operatorname{sect} 5,5: \operatorname{sect} 6,6: \operatorname{sect} 7,7: \text { none }\end{array}$ \\
\hline \multirow[t]{3}{*}{$\begin{array}{l}\text { STOP } \\
\text { SECTOR }\end{array}$} & 1 bit & $\begin{array}{l}\text { 1: Difference detected in stop pulse } \\
\text { numbers deduced from TDC output and } \\
\text { FPGA count. }\end{array}$ \\
\hline & & 0: No difference detected (normal) \\
\hline & 3 bit & $\begin{array}{l}0: \operatorname{sect} 1,1: \operatorname{sect} 2,2: \operatorname{sect} 3,3: \operatorname{sect} 4, \\
4: \operatorname{sect} 5,5: \operatorname{sect} 6,6: \operatorname{sect} 7,7: \operatorname{sect} 8\end{array}$ \\
\hline \multirow[t]{7}{*}{ TOF } & 10 bit & 0x000: This value is not generated (*). \\
\hline & & 0x001-0x3ef: TOF \\
\hline & & 0x3f0-0x3fc: spare \\
\hline & & 0x3fd: No signal on START SECTOR \\
\hline & & 0x3fe: No signal on STOP SECTOR \\
\hline & & 0x3ff: No signal on both START SECTOR \\
\hline & & and STOP SECTOR \\
\hline
\end{tabular}

send all of them to MDP1, where the reported data are the first 136 events per energy step. Therefore, the data packet of the coincidence mode contains counter values in which all of the corresponding events are counted. The counter mode provides the detailed counter 
Table 28 Format of counter mode packet of ENA

\begin{tabular}{|c|c|c|}
\hline \multicolumn{2}{|l|}{$\mathrm{TI}$} & 48 bit \\
\hline \multicolumn{2}{|l|}{ packetID } & 8 bit $(0 \times 01)$ \\
\hline \multicolumn{2}{|l|}{ slotID } & 8 bit \\
\hline \multicolumn{2}{|l|}{$\begin{array}{l}\text { START RING and } \\
\text { START SECTOR } \\
\text { COUNT }\end{array}$} & 448 bit $=4$ ch $\times 7$ ch $\times 16$ bit \\
\hline \multicolumn{2}{|l|}{$\begin{array}{l}\text { START RING } \\
\text { COUNT }\end{array}$} & 64 bit $=4 \mathrm{ch} \times 16$ bit \\
\hline \multicolumn{2}{|l|}{$\begin{array}{l}\text { START SECTOR } \\
\text { COUNT }\end{array}$} & $112 \mathrm{bit}=7 \mathrm{ch} \times 16 \mathrm{bit}$ \\
\hline \multicolumn{2}{|l|}{$\begin{array}{l}\text { STOP SECTOR } \\
\text { COUNT }\end{array}$} & 128 bit $=8 \mathrm{ch} \times 16$ bit \\
\hline $\begin{array}{l}\text { COINCIDENCE } \\
\text { START SECTOR } \\
\text { COUNT }\end{array}$ & $\begin{array}{l}\text { Incremented when signals from START SECTOR, } \\
\text { START RING, and STOP SECTOR are detected } \\
\text { and TOF is calculated. }\end{array}$ & $112 \mathrm{bit}=7 \mathrm{ch} \times 16$ bit \\
\hline $\begin{array}{l}\text { COINCIDENCE } \\
\text { STOP SECTOR } \\
\text { COUNT }\end{array}$ & $\begin{array}{l}\text { Incremented when signals from START SECTOR, } \\
\text { START RING, and STOP SECTOR are detected } \\
\text { and TOF is calculated. }\end{array}$ & 128 bit $=8 \mathrm{ch} \times 16$ bit \\
\hline TOF event data & & $\mathrm{N} \times 20$ bit \\
\hline Rest & & Filled with zero \\
\hline $\begin{array}{l}\text { TOTAL } \\
(=(1 / 128) * \mathrm{Ts})\end{array}$ & & 3072 bit \\
\hline
\end{tabular}

\footnotetext{
*1 Above table shows a format for 1 energy step

*2 MDP1 will read the data every 8 energy steps
}

data shown in Table 28, although the maximum number of TOF event data sent to MDP1 is limited to 100 for each energy step.

When the ENA sensor is in the engineering mode, housekeeping (HK) and status data are sent to MDP1. On the other hand, the reference table of sweeping high voltages (SVs) for the energy scan is sent via the table read mode.

\section{Data processing in MDP1}

The MDP1 receives 24576 bit $=3072$ byte/read cycles, which includes scientific data but excludes HK data. The contents of the scientific data change according to the sensor mode. After receiving the data, MDP1 decodes and reformats both types of data. The format of the data is dependent on the telemetry mode. All telemetry data transferred to the S/C must have timing information added. The time-tagging provides the acquisition time of the data.

The instrument has four telemetry modes that define the data format and data processing before transmission to ground. The MDP1 processes the data collected from the sensor and generates data sets compatible with one of the four telemetry modes. The data sets are either down-linked to ground or are stored in temporary memory on the S/C side. The telemetry modes are given below.

(a) Mass accumulation mode

(b) TOF accumulation mode

(c) Count accumulation mode

(d) Non process mode (The sensor data are downlinked with no processing in MDP1)

(e) Idle mode (No telemetry data are generated) 
Table 29 Possible combinations of the telemetry mode with the sensor mode of ENA

\section{Telemetry Mode}

Mass Accumulation Mode

TOF Accumulation mode

Count Accumulation Mode

Non-Process Mode

Idle Mode
Sensor Mode

Coincidence Mode

Coincidence Mode

Counter Mode

Any

Any

There are limitations for possible combinations of the telemetry mode and the sensor mode. Table 29 summarizes the allowable combinations.

In the Mass, TOF, and Count accumulation modes, the memory in the MDP1 is allocated for count data accumulation. Data originating from the sensor is sorted by look-up tables and is categorized into two types of accumulation matrices during a time period specified by commands. One is the accumulation matrix as described below.

\section{Contents:}

Event data integrated during a sampling period

\section{Dimensions:}

Four dimensions (E, P, C, M) for the mass accumulation mode,

Two dimensions (E, TOF) for the TOF accumulation mode,

Three dimensions (E, P, X) for the count accumulation mode,

where $\mathrm{E}$ is the energy group, $\mathrm{C}$ is the channel group, $\mathrm{P}$ is the phase group, $\mathrm{M}$ is the mass group, TOF is time of flight, and $\mathrm{X}$ is the type of counters, namely START ring, START sector, STOP plate, STOP coincidence, or START coincidence count. The other matrix is the accumulation scaling matrix, as described below.

\section{Contents:}

Counter data summed during a sampling period

\section{Dimensions:}

Three dimensions (E, P, Y) for the mass accumulation mode,

Two dimensions (E, Y) for TOF accumulation mode,

Not needed for the count accumulation mode,

where $\mathrm{Y}$ is the type of counters, namely total START count, total STOP count, coincidence STOP count, coincidence START and STOP counters (seven + eight). The numbers of bins in each element can be set by commands. Possible numbers of bins are given below.

$\mathrm{n}(\mathrm{C}) 1$ or 7 ;

n(E) $1,2,4$, or 8 ;

$\mathrm{n}(\mathrm{P}) \mathrm{1}, 2,4,8,16$, or 32 ;

$\mathrm{n}(\mathrm{M}) 1,2,4,8,16,32,64$ or 128 ;

n(TOF) 1024;

where $\mathrm{n}(\mathrm{XX})$ means the number of bins for $\mathrm{XX}$. The numbers of bins in $\mathrm{E}$ and $\mathrm{P}$ are coupled and are not independent:

If $n(E)=1,2$ or 4 then $n(P)=1,2,4,8,16$, or 32

If $n(E)=8$ then $n(P)=1,2,4,8$ or 16 


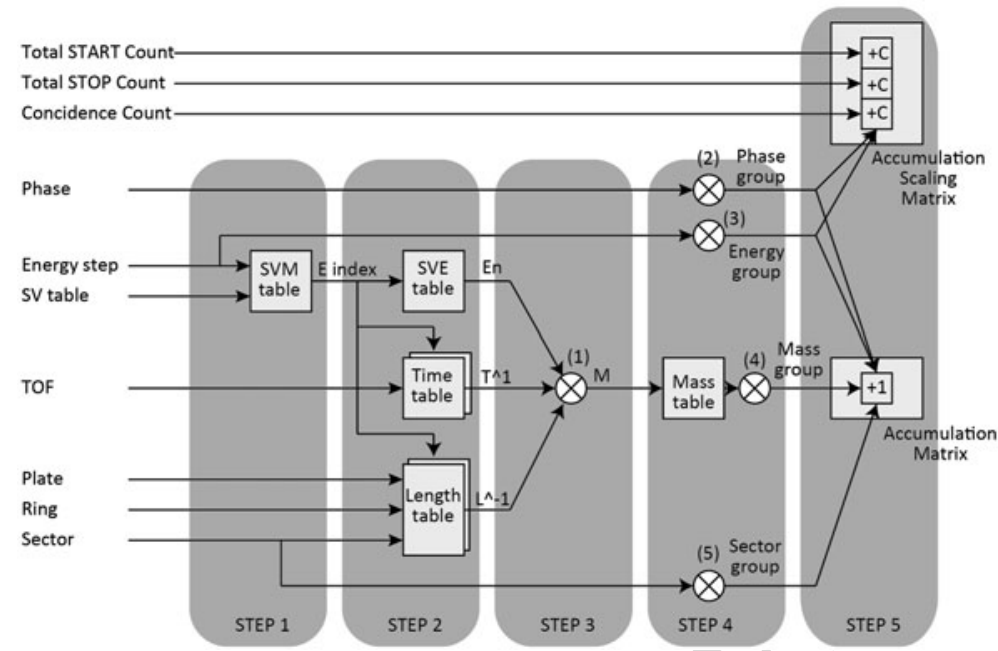

Fig. 80 Schematic diagram of the calculation flow in mass accumulation mode

The total number of elements in the accumulation matrix should not exceed 8192 owing to memory space limitations. Any mode requiring a larger accumulation matrix is invalid.

\section{Mass accumulation mode}

This mode is used to obtain ENA data with mass information and will commonly be used in the orbit. This telemetry mode requires the coincidence mode as the sensor mode. Coincidence data from the sensor are sorted and accumulated into a matrix. The accumulation matrix has four dimensions: $\mathrm{E}$ (energy), $\mathrm{C}$ (channel), $\mathrm{P}$ (phase), and M (mass). An accumulation is made during a specified time interval, which is defined by a command. To compensate for high count rates, where not enough space is available to transmit all coincidence events, the total number of START, STOP, and coincidence STOP counts is added up for each combination of $n(P)$ and $n(E)$ in the accumulation scaling matrix. This matrix enables scaling of the accumulation matrix during the data analysis on ground. When the mass accumulation mode is set, the coincidence mode is always needed as the sensor mode. In this case MDP1 receives event entries of coincidence counts in each energy step. It then obtains its mass group (M), incoming direction (C), and energy (E) for every coincidence event and accumulates them into the accumulation matrix. This accumulation is made over a certain time set by a command. Finally, MDP1 sends to S/C the contents of the accumulation matrix and other information after the accumulation.

A schematic data calculation flow diagram of the mass accumulation mode is shown in Fig. 80. A mass group M is calculated from the START ring, START sector, STOP plate and TOF by using look-up tables. The energy group $\mathrm{E}$ as well as the phase group $\mathrm{P}$ are calculated from the slot number of each sensor data packet. The channel group C corresponds directly to START sector. For every event obtained, the MDP1 increments the counters specified by $\mathrm{E}, \mathrm{P}, \mathrm{C}$ and $\mathrm{M}$ in the accumulation matrix. The numbers of bins for each parameter can be set by commands. The total counter data included in sensor data are accumulated in the accumulation scaling matrix. This matrix only has two dimensions; energy group $\mathrm{E}$ and phase group $\mathrm{P}$. 


\section{MDP1 Data processing method on the mass accumulation mode}

Data processing on MDP1 for each event consists of five steps, as given below.

\section{Step 1 Obtain index of energy depending on sweep pattern}

This index will later be used to obtain the actual value of the energy from the SVE table

\section{Energy-index:}

E-index $=$ SVM-Table[SV-index, Energy-step $]$,

where SV-index is selected from table ID for the SV reference table during the observation.

\section{Step 2 Obtain derived data for mass calculation by look-up tables}

(a) Square root of energy: En = SVE-Table [E-index] (10 bit values)

(b) Inv. of path length: $\mathrm{L}_{\text {inv }}=\mathrm{LT}$ [sector, ring, plate, E-index] (12 bit values)

(c) Flight time: $\mathrm{t}_{1}=\mathrm{TT}$ [tof] (10 bit values)

The SVE-table returns the actual particle energy corresponding to an Energy-index. Consequently, the values returned from the SVM- and SVE-tables must reflect the particle energy selected in the $\mathrm{SV}$ table. If $\mathrm{L}_{\text {inv }}$ is zero, it is regarded as an invalid event by the onboard software. When an anomaly is found on a specific START SECTOR-START RINGSTOP SECTOR pair at a specific energy, it can be rejected from the onboard calculation by setting $\mathrm{L}_{\mathrm{inv}}=0$ on the LT-table. Therefore, the LT-table is designed as energy dependent. The LT-table can also compensate for a possible energy dependent energy loss on the START surface. In this case, the tof-path-length used in the mass calculation would be artificially extended for energies that have a higher relative energy loss. This was not used, however, based on the calibration results. Therefore all the path lengths used from the LT-table were the same for all energies. The TT-table returns the tof value only when tof $\leq 0 \times 3$ ef. If tof $\geq 0 \times 3 f 0$, it returns 0 which is regarded as invalid event in the onboard calculation (Table 27).

\section{Step 3 Calculate mass}

Mass calculation is accomplished using 32 bit unsigned integer operations. The values of the tables in step 2 guarantee that no overflow occurs.

$$
\text { mass_0 } 5=\left(\left\{\left(E n \times t_{1} \times L_{\text {inv }}\right) / 65536\right\} \times \text { Factor }\right) / 65536
$$

where Factor $=3340$. If the calculated value for mass_0_5 is larger than 255 , a value of 255 must be assigned to mass_0_5 prior to further processing. The value calculated here is actually proportional to the square root of the mass.

\section{Step 4 Bin the data according to the binning parameters set}

$$
\text { (a) mass group } \mathrm{M}=\mathrm{MT}[\text { mass_0_5] } /\{128 / \mathrm{n}(\mathrm{M})\}
$$

(b) channel group $\mathrm{C}=\operatorname{sector} /\{7 / \mathrm{n}(\mathrm{C})\}$

(c) energy group $\mathrm{E}=$ Energy_step modulo $\mathrm{n}(\mathrm{E})$

(d) phase group $\mathrm{P}=$ (phase - phasemin $) /\{32 / \mathrm{n}(\mathrm{P})\}$

Where phasemin contains the first spin phase value to be considered, whereas phasemax is the last phase value to be considered. To use all phase values, phasemin $=0$, phasemax $=$ 31. All divisions in equations (2)? (5) are integer divisions. 


\section{Step 5 Update the accumulation and the accumulation scaling matrix}

Accumulation matrix $(\mathrm{M}, \mathrm{C}, \mathrm{E}, \mathrm{P})=$ Accumulation matrix $(\mathrm{M}, \mathrm{C}, \mathrm{E}, \mathrm{P})+1$

Accumulation scaling matrix $(\mathrm{E}, \mathrm{P}, \mathrm{Y})=$ Accumulation matrix $(\mathrm{E}, \mathrm{P}, \mathrm{Y})+1$

This calculation is required for all events in a packet.

\section{Count accumulation mode}

This mode is used to obtain the detailed signal count at each MCP plate and is similar to the mass accumulation mode, except that counter data are summed. When count accumulation mode is set, the sensor always needs to be set to the counter mode. In this mode, instead of receiving coincidence events, MDP1 receives detailed total counts on each MCP anode during one energy step. Then the total count data are summed as the accumulation matrix. This accumulation is made over a particular time set by a command, and the contents of the accumulation matrix are sent to $\mathrm{S} / \mathrm{C}$.

\section{TOF accumulation mode}

This mode is used to obtain raw TOF distributions without mass information. The primary uses of the raw TOF data are to analyze the performance of the instrument and to calibrate the on-board mass calculations. This mode is similar to the mass accumulation mode, except that TOF data are accumulated instead of mass data. In TOF accumulation mode, the mass is not calculated, and the raw TOF data are directly used for the accumulation instead of the mass. The accumulation matrix in this mode has two dimensions: $\mathrm{E}$ (energy) and $\mathrm{T}$ (TOF). MDP1 does not use the other parameters of $\mathrm{P}$ (phase) and $\mathrm{C}$ (channel). Because TOF data have 10 bits, the number of TOF bins is always 1024 .

\subsubsection{Pre-flight Calibration of ENA}

The instrument was calibrated at the Messkammer für Flugzeitinstrumente und Time-offlight (MEFISTO) calibration facility (Marti et al. 2001) at the University of Bern. The facility produces an energetic neutral atom beam by neutralizing a collimated ion beam on a conversion surface (Wieser and Wurz 2005). The beam neutralizer is an integral part of the MEFISTO facility and produces a neutral beam of known composition, well-characterized in angle and energy. For the instrument calibration, neutral $\mathrm{H}$, neutral $\mathrm{He}$ and neutral $\mathrm{O}$ beams were produced in an energy range from $30-3000 \mathrm{eV}$ per particle. During the calibration the instrument was mounted on the MEFISTO hexapod turntable, which enabled rotation and translation of instrument relative to the fixed energetic neutral atom beam. An additional thermal heating/cooling plate enabled performance investigation at different temperatures (Fig. 81).

The calibration was split into four phases between 2012 and 2014 with interspersed calibration data analysis phases. This approach enabled to repeat measurements that had insufficient statistics and other quality problems detected during the data analysis. The calibration tasks were separated into establishing energy response, angular response and mass response.

\section{Energy response}

The energy response of ENA is determined by the electrostatic wave energy analysis system and the energy loss function on the conversion surface. The latter strongly depends on species and energy, which makes the energy response species and energy dependent. To simplify operations, only 16 different energy settings were characterized, with each identified 
Fig. 81 ENA at the MEFISTO calibration facility. The neutral beam is emitted from the beam neutralizer on the left (gold box). ENA (center) is encapsulated in thermal insulation sheets to facilitate temperature control. The entire instrument setup can be moved and rotated relative to the incoming neutral beam with $6^{\circ}$ of freedom

Table 30 Calibrated energy bin centers for hydrogen and oxygen

\begin{tabular}{llll}
\hline E-Index & $\begin{array}{l}\text { Nominal center } \\
\text { energy }(\mathrm{eV})\end{array}$ & $\begin{array}{l}\text { Hydrogen } \\
(\mathrm{eV})\end{array}$ & $\begin{array}{l}\text { Oxygen } \\
(\mathrm{eV})\end{array}$ \\
\hline
\end{tabular}

\begin{tabular}{rrrr}
\hline 0 & 0 & 0 & 0 \\
1 & 10 & 20 & 8 \\
2 & 20 & 37 & 15 \\
3 & 40 & 67 & 29 \\
4 & 80 & 120 & 54 \\
5 & 160 & 215 & 103 \\
6 & 320 & 387 & 196 \\
7 & 640 & 693 & 370 \\
8 & 1280 & 1243 & 699 \\
9 & 2560 & 2228 & 1321 \\
10 & 56 & 89 & 39 \\
11 & 112 & 159 & 74 \\
12 & 224 & 286 & 141 \\
13 & 448 & 513 & 267 \\
14 & 896 & 920 & 504 \\
15 & 1792 & 1650 & 953 \\
\hline
\end{tabular}

by an index and nominal center energy for bookkeeping purposes. The actual peak energy of each energy bin is species dependent (Table 30). The actual energy sweep in the instrument consisted of eight energy settings selected from this table. The width of the energy pass band $\Delta \mathrm{E}$ for hydrogen is energy independent with $\Delta \mathrm{E} / \mathrm{E}=100 \%$, with $\mathrm{E}$ as the nominal center energy. The energy pass bands for oxygen have a tail toward higher energies owing to the more prominent energy loss at the conversion surface. The quantitative extent of this effect needs further analysis. 
Fig. 82 Typical mass spectra for incident neutral hydrogen (top) and oxygen (bottom). The black lines show the measured data, the colored lines are fitted curves. $\mathrm{H}^{0}$ : hydrogen peak, $\mathrm{O}^{0}$ : oxygen peak, $\mathrm{H}^{0} \mathrm{r}_{\mathbf{c} / \mathbf{s}}$ : hydrogen signal owing to a recoil process at the conversion surface. The $\mathrm{X}$-axis in both plots is in mass bin units $\mathrm{M}$ as shown in Fig. 80 and the $\mathrm{Y}$-axis is in arbitrary units

Table 31 Calibrated angular response

\section{Angular response}

The angular response was determined by rotating the instrument relative to an incident neutral hydrogen beam in a grid like pattern. The count rates obtained from each START sector were then fitted with 2D angular Gaussian profiles. Table 31 shows the bore sight directions and widths of each of the seven viewing directions corresponding to the seven START sectors. $\theta_{0}$ denotes the center in azimuth, $\beta_{0}$ is the center in the elevation direction, and $\Delta \theta$ and $\Delta \beta$ are the FWHM values of the fitted peak widths. The coordinate system used is shown in Fig. 72. The azimuthal resolution $\Delta \theta$ varies linearly between $32.5^{\circ}$ at sector 0 and $22.9^{\circ}$ at sector 6 , and the elevation resolution $\Delta \beta$ decreases linearly between $11.6^{\circ}$ at sector 0 and $7.1^{\circ}$ at sector 6 . We believe this trend to be a result of a mechanical misalignment between the wave system and the TOF cell. The angular response is in first order not energy dependent.

\section{Mass response}

The TOF values measured onboard are converted to a nearly energy independent mass number $\mathrm{M}$ in the range from $0-255$. Before reporting to telemetry, $\mathrm{M}$ is converted to a mass group number compatible with the selected binning parameters by using a mass lookup

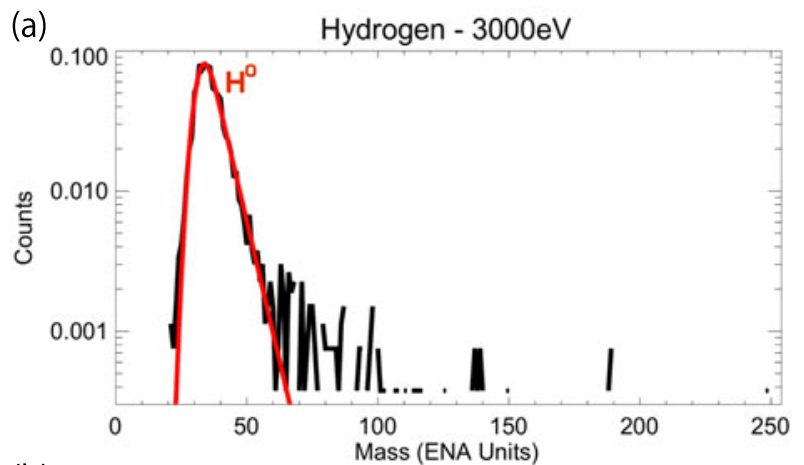

(b) Oxygen, $3000 \mathrm{eV}$

\begin{tabular}{llllllrr}
\hline Start sector & 0 & 1 & \multicolumn{1}{c}{2} & \multicolumn{1}{c}{3} & \multicolumn{1}{c}{4} & \multicolumn{1}{c}{5} & \multicolumn{1}{c}{6} \\
\hline$\theta_{0}\left({ }^{\circ}\right)$ & 67.1 & 40.8 & 16.9 & -3.0 & -24.2 & -46.3 & -68.1 \\
$\beta_{0}\left({ }^{\circ}\right)$ & -5.4 & -5.8 & -6.5 & -5.3 & -5.8 & -5.6 & -5.2 \\
$\Delta \theta\left(^{\circ}\right)$ & 32.8 & 28.9 & 25.8 & 27.0 & 21.8 & 21.5 & 22.9 \\
$\Delta \beta\left(^{\circ}\right)$ & 11.6 & 10.5 & 9.9 & 9.0 & 7.7 & 7.8 & 7.1 \\
\hline
\end{tabular}


Table 32 Selected estimated geometric factors not including detection probability for neutral hydrogen. Values indicated with $\mathrm{n} / \mathrm{a}$ are not yet available

\begin{tabular}{rrl}
$\begin{aligned} \text { E-Index Nominal center } \\
\text { energy }(\mathrm{eV})\end{aligned}$ & $\begin{array}{l}\text { G for hydrogen without detection probability } \\
\left(\mathrm{cm}^{2} \mathrm{sreV} / \mathrm{eV}\right)\end{array}$ \\
\hline 0 & 0 & $\mathrm{n} / \mathrm{a}$ \\
1 & 10 & $\mathrm{n} / \mathrm{a}$ \\
2 & 20 & $\mathrm{n} / \mathrm{a}$ \\
3 & 40 & $0.9 \times 10^{-7}$ \\
4 & 80 & $1.2 \times 10^{-7}$ \\
5 & 160 & $1.9 \times 10^{-7}$ \\
6 & 320 & $2.5 \times 10^{-7}$ \\
7 & 640 & $0.5 \times 10^{-6}$ \\
8 & 1280 & $\mathrm{n} / \mathrm{a}$ \\
9 & 2560 & $\mathrm{n} / \mathrm{a}$ \\
10 & 56 & $1.0 \times 10^{-7}$ \\
11 & 112 & $\mathrm{n} / \mathrm{a}$ \\
12 & 224 & $\mathrm{n} / \mathrm{a}$ \\
13 & 448 & $3.6 \times 10^{-7}$ \\
14 & 896 & $\mathrm{n} / \mathrm{a}$ \\
15 & 1792 & $\mathrm{n} / \mathrm{a}$ \\
\hline
\end{tabular}

table and possible further division by using a constant, as shown in Fig. 80. For calibration data analysis the mass number $\mathrm{M}$ was used to establish the shapes of the different mass peaks in the mass spectrum. The shape of the hydrogen mass peak is shown in Fig. 82(a). Owing to its larger mass, oxygen might generate hydrogen recoils at the conversion surface, which can result in an additional hydrogen peak even when the conversion surface is hit by oxygen only (Fig. 82(b)). The intensity of the additional hydrogen signal depends mainly on the amount of water absorbed on the conversion surface. The mass spectrum to neutral helium is more complicated because both recoil hydrogen and recoil oxygen atoms appear. For the case of incident neutral oxygen, a mass comprehensive cross-talk matrix could be generated, which enables separation of the individual recoil contributions. The matrix for incident neutral helium is sparser owing to limited calibration time available for helium.

\section{Geometric factor}

In its simplest form the geometric factor $\mathrm{G}$ is expressed as

$$
\mathrm{G}=\Delta \mathrm{E} / \mathrm{E} \times \Delta \Omega \times \Delta \mathrm{A} \times \epsilon
$$

where $\Delta \mathrm{E} / \mathrm{E}$ is the energy resolution, $\Delta \mathrm{A}$ is the effective aperture area, $\Delta \Omega=\Delta \theta \times \Delta \beta$ is the angular acceptance, and $\epsilon$ is the detection probability. The first two factors energy response and angular response are well established. The detection probability for both START and STOP detectors is obtained from the START and STOP rates in the TOF cell (Funsten et al, 2005). Typical observed values for hydrogen are $1 \%$ at $100 \mathrm{eV}$ increasing to $30 \%$ at $1000 \mathrm{eV}$. The best estimates for the geometric factor for neutral hydrogen are listed in Table 32.

\subsubsection{Near-Earth Commissioning Results of ENA}

The temperature of the conversion surface is controlled to remain above $+50{ }^{\circ} \mathrm{C}$ throughout the mission to avoid a stack of contaminating materials which would decrease the efficiency 
Table 33 MPPE data mode and L-mode data products of LEP and HEP sensors

\begin{tabular}{|c|c|}
\hline MPPE mode name & L-mode data products \\
\hline 1. Default Observation Mode & $\begin{array}{l}\text { MEA1: Et-OMN, Et-PAP, VM, 3D-LL } \\
\text { MEA2: Et-OMN, Et-PAP, VM } \\
\text { MIA: Et-M1, VM-M1, 3D-LL-M1 } \\
\text { MSA: Low [Moments, Omni E-t, TOF] } \\
\text { HEP: L-mode }\end{array}$ \\
\hline 2. Exospheric Mode & $\begin{array}{l}\text { MEA1: Et-OMN, Et-PAP, VM, 3D-LL } \\
\text { MEA2: Et-OMN, Et-PAP, VM } \\
\text { MIA: Et-M2, VM-M2, 3D-LL-M2 } \\
\text { MSA: Low [Moments, Omni E-t, TOF] } \\
\text { HEP: L-mode }\end{array}$ \\
\hline 3. Solar Wind Mode/IP Shock Local Mode & $\begin{array}{l}\text { MEA1: Et-OMN, Et-PAP, VM, 3D-LL } \\
\text { MEA2: Et-OMN, Et-PAP, VM } \\
\text { MIA: Et-M1, VM-M1, 3D-LL-M1 } \\
\text { MSA: Low [Moments, Omni E-t, TOF] } \\
\text { HEP: L-mode }\end{array}$ \\
\hline 4. IP Shock Macro Mode/Bow Shock Mode & $\begin{array}{l}\text { MEA1: Et-OMN, Et-PAP, VM, 3D-LL } \\
\text { MEA2: Et-OMN, Et-PAP, VM } \\
\text { MIA: Et-M1, VM-M1, 3D-LL-M1 } \\
\text { MSA: Low [Moments, Omni E-t, TOF] } \\
\text { HEP: L-mode }\end{array}$ \\
\hline 5. Reconnection Mode & $\begin{array}{l}\text { MEA1: Et-OMN, Et-PAP, VM, 3D-LL } \\
\text { MEA2: Et-OMN, Et-PAP, VM } \\
\text { MIA: Et-M3, VM-M3, 3D-LL-M3 } \\
\text { MSA: Low [Moments, Omni E-t, TOF] } \\
\text { HEP: L-mode }\end{array}$ \\
\hline 6. Magnetospheric Mode & $\begin{array}{l}\text { MEA1: Et-OMN, Et-PAP, VM, 3D-LL } \\
\text { MEA2: Et-OMN, Et-PAP, VM } \\
\text { MIA: Et-M3, VM-M3, 3D-LL-M3 } \\
\text { MSA: Low [Moments, Omni E-t, TOF] } \\
\text { HEP: L-mode }\end{array}$ \\
\hline
\end{tabular}

of neutral to ion conversion. So far, the temperature has been successfully maintained above $+50{ }^{\circ} \mathrm{C}$, except for some cases of short duration that did not affect the conversion performance because the temperature was still greater than that of the surrounding structures. The power for the ENA heater control and temperature monitoring system (ENA HCS) is separated from the other ENA electronics.

Except for a high-voltage unit, ENA was activated on November 25, 2018 for the first time after the launch. No problems were found during the test. The ENA function tests with the HVPS were conducted on June 27 and 28, 2019; August 20 and 22, 2019; and February 6 and 7, 2020. These tests were conducted when the spacecraft was in the solar wind. During the testing, all of the high-voltage outputs were gradually increased up to their nominal set- 
Table 34 MPPE data mode and M-mode data products of LEP and HEP sensors

\begin{tabular}{|c|c|}
\hline MPPE mode name & M-mode data products \\
\hline 1. Default Observation Mode & $\begin{array}{l}\text { MEA1: Et-OMNm, Et-PAP, VM, 3D-M } \\
\text { MEA2: Et-OMNm, Et-PAP, VM } \\
\text { MIA: 3D-L2-M1 or SW-L2-M1 } \\
\text { MSA: Med A [Moment, 3D-VDF(A), AD(A), TOF(A)] } \\
\text { HEP: M-mode }\end{array}$ \\
\hline 2. Exospheric Mode & $\begin{array}{l}\text { MEA1: Et-OMNm, Et-PAP, VM, 3D-M } \\
\text { MEA2: Et-OMNm, Et-PAP, VM } \\
\text { MIA: 3D-L2-M2 } \\
\text { MSA: Med D [Moment, 3D-VDF(D), AD(D), TOF(D)] } \\
\text { HEP: M-mode }\end{array}$ \\
\hline 3. Solar Wind Mode/IP Shock Local Mode & $\begin{array}{l}\text { MEA1: Et-OMNm, Et-PAP, VM, 3D-M } \\
\text { MEA2: Et-OMNm, Et-PAP, VM } \\
\text { MIA: 3D-L2-M1 or SW-L2-M1 } \\
\text { MSA: Med C [Moment, 3D-VDF(C), AD(C), TOF(C)] } \\
\text { HEP: M-mode }\end{array}$ \\
\hline 4. IP Shock Macro Mode/Bow Shock Mode & $\begin{array}{l}\text { MEA1: Et-OMNm, Et-PAP, VM, 3D-M } \\
\text { MEA2: Et-OMNm, Et-PAP, VM } \\
\text { MIA: 3D-L2-M1 or SW-L2-M1 } \\
\text { MSA: Med C [Moment, 3D-VDF(C), AD(C), TOF(C)] } \\
\text { HEP: M-mode }\end{array}$ \\
\hline 5. Reconnection Mode & $\begin{array}{l}\text { MEA1: Et-OMNm, Et-PAP, VM, 3D-M } \\
\text { MEA2: Et-OMNm, Et-PAP, VM } \\
\text { MIA: 3D-L2-M3 } \\
\text { MSA: Med C [Moment, 3D-VDF(C), AD(C), TOF(C)] } \\
\text { HEP: M-mode }\end{array}$ \\
\hline 6. Magnetospheric Mode & $\begin{array}{l}\text { MEA1: Et-OMNm, Et-PAP, VM, 3D-M } \\
\text { MEA2: Et-OMNm, Et-PAP, VM } \\
\text { MIA: 3D-L2-M3 } \\
\text { MSA: Med D [Moment, 3D-VDF(D), AD(D), TOF(D)] } \\
\text { HEP: M-mode }\end{array}$ \\
\hline
\end{tabular}

tings for the actual observation. For the input surface of START and STOP MCPs, $-2300 \mathrm{~V}$ and $-2600 \mathrm{~V}$ were applied, respectively, where dark counts were successfully detected by all input channels of the pre-amplifiers. For the other electrodes, voltage sweeping for energy analysis was also tested with no problems detected. However, no valid ENA counts were identified during the testing, because the FOV of ENA is mostly blocked by MOSIF during the cruising phase. Although part of the FOV of ENA is not blocked by MOSIF, the unblocked direction is not pointed toward the Sun. In this case, faint ENA flux is expected in the solar wind. 
Table 35 MPPE data mode and H-mode data products of LEP and HEP sensors

\begin{tabular}{|c|c|}
\hline MPPE mode name & H-mode data products \\
\hline 1. Default Observation Mode & N.A. \\
\hline \multirow[t]{5}{*}{ 2. Exospheric Mode } & MEA1: 3D-H \\
\hline & MEA2: 3D-H \\
\hline & MIA:3D-H-M2 \\
\hline & MSA: High B [Moment, 3D-VDF(B), TOF(B), EVENT(B)] \\
\hline & HEP: H-mode \\
\hline \multirow[t]{5}{*}{ 3. Solar Wind Mode/IP Shock Local Mode } & MEA1: 3D-H \\
\hline & MEA2: 3D-H \\
\hline & MIA: SW-L-M1, 3D-L2-M1 \\
\hline & MSA: High B [Moment, 3D-VDF(B), TOF(B), EVENT(B)] \\
\hline & HEP: H-mode \\
\hline \multirow[t]{5}{*}{ 4. IP Shock Macro Mode/Bow Shock Mode } & MEA1: 3D-H \\
\hline & MEA2: 3D-H \\
\hline & MIA: SW-L-M1, 3D-L2-M1 \\
\hline & MSA: High B [Moment, 3D-VDF(B), TOF(B), EVENT(B)] \\
\hline & HEP: H-mode \\
\hline \multirow[t]{5}{*}{ 5. Reconnection Mode } & MEA1: 3D-H \\
\hline & MEA2: 3D-H \\
\hline & MIA: 3D-H-M3 \\
\hline & MSA: High B [Moment, 3D-VDF(B), TOF(B), EVENT(B)] \\
\hline & HEP: H-mode \\
\hline \multirow[t]{5}{*}{ 6. Magnetospheric Mode } & MEA1: 3D-H \\
\hline & MEA2: 3D-H \\
\hline & MIA: 3D-H-M3 \\
\hline & MSA: High B [Moment, 3D-VDF(B), TOF(B), EVENT(B)] \\
\hline & HEP: H-mode \\
\hline
\end{tabular}

ENA will be activated during the Earth and Venus fly-bys before the Mercury orbit insertion to measure ENAs generated by charge-exchange interactions between hot ions and cold atmospheric neutrals.

\section{Data Products of MPPE}

To conduct coordinated observation between the MPPE sensors and to control the total telemetry data rate, the MPPE data mode is defined. The operation mode of LEP and HEP sensors are determined so that they generate the data products depending on the MPPE data mode. Because ENA generates only L-mode data with fixed data rates, it is operated independently of the MPPE data mode. Six MPPE data modes are defined: default observation mode, exospheric mode, solar wind mode/IP shock local mode, IP shock macro mode/bow shock mode, reconnection mode, and magnetospheric mode. Tables 33, 34, 35 show the 
MPPE data mode and the corresponding data products of LEP and HEP sensors for L-mode, M-mode, and H-mode data, respectively.

\section{Conclusion}

All of the MPPE analyzers have concluded initial commissioning with no significant problems reported. Because MOSIF blocks most of the FOVs of the MPPE sensors, it will be difficult for the ion sensors (MIA, MSA, and HEP-ion) to measure the solar wind during the cruise phase before arriving at Mercury. Only the low energy electron sensors MEA1 and MEA2 can measure part of the solar wind electron phase space density because the thermal speed of electrons is higher than the solar wind bulk velocity. During the Earth, Venus, and Mercury fly-bys, we expect most of the MPPE sensors to be turned on. If BepiColombo will pass through magnetosphere, the ion sensors might also be able to measure natural counts. Therefore, events provide good opportunities to check the analyzer functions including the data processing software using natural data.

During the Venus fly-bys scheduled in October 2020 and August 2021, we plan to use MEA1, MEA2, MIA, MSA, HEP-ele, and ENA to make observations. MEA1 and MEA2 will be able to observe electrons in the solar wind and around Venus, and ENA might be capable of measuring the energetic neutral atoms from Venus. Although it may be difficult for MIA and MSA to obtain meaningful ion data, activating the instrument and operating the analyzers will refresh the instrument operation skills and facilitate observation immediately after arriving at Mercury. During the Mercury fly-bys scheduled in October 2021, June 2022, June 2023, September 2024, December 2024 and January 2025, the MPPE sensors will be activated; detailed observation plans will be considered in the future. After arriving at Mercury in December 2025, all of the MPPE analyzers will make continuous observations except for periods in which the operations are limited owing to the thermal constraints of the spacecraft.

Acknowledgements The authors thank all the members of BepiColombo mission for their unstinted efforts in making the mission fruitful and for their valuable discussion on the specifications and operations of payload instruments including our MPPE sensors.

\section{Declarations}

Conflict of Interest The authors declare that they have no conflict of interest.

\section{References}

T. Amano, T. Katou, N. Kitamura, M. Oka, Y. Matsumoto, M. Hoshino, Y. Saito, S. Yokota, B.L. Giles, W.R. Paterson, C.T. Russell, O. Le Contel, R.E. Ergun, P.A. Lindqvist, D.L. Turner, J.F. Fennell, J.B. Blake, Observational evidence for stochastic shock drift acceleration of electrons at the Earth's bow shock. Phys. Rev. Lett. 124, 065,101 (2020). https://doi.org/10.1103/PhysRevLett.124.065101

B.J. Anderson, C.L. Johnson, H. Korth, M.E. Purucker, R.M. Winslow, J.A. Slavin, S.C. Solomon, R.L. McNutt, J.M. Raines, T.H. Zurbuchen, The global magnetic field of Mercury from MESSENGER orbital observations. Science 333(6051), 1859-1862 (2011). https://doi.org/10.1126/science.1211001

B.J. Anderson, C.L. Johnson, H. Korth, J.A. Slavin, R.M. Winslow, R.J. Phillips, R.L. McNutt Jr., S.C. Solomon, Steady-state field-aligned currents at Mercury. Geophys. Res. Lett. 41(21), 7444-7452 (2014). https://doi.org/10.1002/2014GL061677 
S. Barabash, R. Lundin, H. Andersson, K. Brinkfeldt, A. Grigoriev, H. Gunell, M. Holmström, M. Yamauchi, K. Asamura, P. Bochsler, P. Wurz, R. Cerulli-Irelli, A. Mura, A. Milillo, M. Maggi, S. Orsini, A.J. Coates, D.R. Linder, D.O. Kataria, C.C. Curtis, K.C. Hsieh, B.R. Sandel, R.A. Frahm, J.R. Sharber, J.D. Winningham, M. Grande, E. Kallio, H. Koskinen, P. Riihelä, W. Schmidt, T. Säles, J.U. Kozyra, N. Krupp, J. Woch, S. Livi, J.G. Luhmann, S. McKenna-Lawlor, E.C. Roelof, D.J. Williams, J.A. Sauvaud, A. Fedorov, J.J. Thocaven, The Analyzer of Space Plasmas and Energetic Atoms (ASPERA-3) for the Mars Express mission. Space Sci. Rev. 126(1), 113-164 (2006). https://doi.org/ 10.1007/s11214-006-9124-8

S. Barabash, J.A. Sauvaud, H. Gunell, H. Andersson, A. Grigoriev, K. Brinkfeldt, M. Holmström, R. Lundin, M. Yamauchi, K. Asamura, W. Baumjohann, T. Zhang, A. Coates, D. Linder, D. Kataria, C. Curtis, K. Hsieh, B. Sandel, A. Fedorov, C. Mazelle, J.J. Thocaven, M. Grande, H.E. Koskinen, E. Kallio, T. Säles, P. Riihela, J. Kozyra, N. Krupp, J. Woch, J. Luhmann, S. McKenna-Lawlor, S. Orsini, R. Cerulli-Irelli, M. Mura, M. Milillo, M. Maggi, E. Roelof, P. Brandt, C. Russell, K. Szego, J. Winningham, R. Frahm, J. Scherrer, J. Sharber, P. Wurz, P. Bochsler, The Analyser of Space Plasmas and Energetic Atoms (ASPERA-4) for the Venus Express mission. Planet. Space Sci. 55(12), 1772-1792 (2007). https://doi.org/10.1016/j.pss.2007.01.014. The Planet Venus and the Venus Express Mission, Part 2

T.A. Cassidy, A.W. Merkel, M.H. Burger, M. Sarantos, R.M. Killen, W.E. McClintock, R.J. Vervack, Mercury's seasonal sodium exosphere: MESSENGER orbital observations. Icarus 248, 547-559 (2015). https://doi.org/10.1016/j.icarus.2014.10.037

E.W. Cliver, J. Feynman, H.B. Garrett, An estimate of the maximum speed of the solar wind, 1938-1989. J. Geophys. Res. Space Phys. 95(A10), 17,103-17,112 (1990). https://doi.org/10.1029/ JA095iA10p17103

D.C. Delcourt, S. Grimald, F. Leblanc, J.J. Berthelier, A. Millilo, A. Mura, S. Orsini, T.E. Moore, A quantitative model of the planetary $\mathrm{Na}^{+}$contribution to Mercury's magnetosphere. Ann. Geophys. 21(8), 1723-1736 (2003). https://doi.org/10.5194/angeo-21-1723-2003

D. Delcourt, Y. Saito, F. Leblanc, C. Verdeil, S. Yokota, M. Fraenz, H. Fischer, B. Fiethe, B. Katra, D. Fontaine, J.M. Illiano, J.J. Berthelier, N. Krupp, U. Buhrke, F. Bubenhagen, H. Michalik, The Mass Spectrum Analyzer (MSA) on board the BepiColombo MMO. J. Geophys. Res. Space Phys. 121(7), 6749-6761 (2016). https://doi.org/10.1002/2016JA022380

M.I. Desai, G.M. Mason, M.E. Wiedenbeck, C.M.S. Cohen, J.E. Mazur, J.R. Dwyer, R.E. Gold, S.M. Krimigis, Q. Hu, C.W. Smith, R.M. Skoug, Spectral properties of heavy ions associated with the passage of interplanetary shocks at 1 AU. Astrophys. J. 611(2), 1156-1174 (2004). https://doi.org/10.1086/422211

R.M. Dewey, J.A. Slavin, J.M. Raines, D.N. Baker, D.J. Lawrence, Energetic electron acceleration and injection during dipolarization events in Mercury's magnetotail. J. Geophys. Res. Space Phys. 122(12), 12,170-12,188 (2017). https://doi.org/10.1002/2017JA024617

G.A. DiBraccio, J.A. Slavin, S.M. Imber, D.J. Gershman, J.M. Raines, C.M. Jackman, S.A. Boardsen, B.J. Anderson, H. Korth, T.H. Zurbuchen, R.L. McNutt, S.C. Solomon, MESSENGER observations of flux ropes in Mercury's magnetotail. Planet. Space Sci. 115, 77-89 (2015). https://doi.org/10.1016/j. pss.2014.12.016. Solar wind interaction with the terrestrial planets

S. Fatemi, A.R. Poppe, S. Barabash, Hybrid simulations of solar wind proton precipitation to the surface of Mercury. J. Geophys. Res. Space Phys. 125(4), e2019JA027706 (2020). https://doi.org/10.1029/ 2019JA027706

L.A. Fisk, G. Gloeckler, Particle acceleration in the heliosphere: implications for astrophysics. Space Sci. Rev. 173(1), 433-458 (2012). https://doi.org/10.1007/s11214-012-9899-8

N.J. Fox, M.C. Velli, S.D. Bale, R. Decker, A. Driesman, R.A. Howard, J.C. Kasper, J. Kinnison, M. Kusterer, D. Lario, M.K. Lockwood, D.J. McComas, N.E. Raouafi, A. Szabo, The Solar Probe Plus mission: humanity's first visit to our star. Space Sci. Rev. 204(1), 7-48 (2016). https://doi.org/10.1007/s11214015-0211-6

H.O. Funsten, S.M. Ritzau, R.W. Harper, Negative helium ions exiting a carbon foil at keV energies. Phys. Rev. B 63, 155,416 (2001). https://doi.org/10.1103/PhysRevB.63.155416

H.O. Funsten, R.W. Harper, D.J. McComas, Absolute detection efficiency of space-based ion mass spectrometers and neutral atom imagers. Rev. Sci. Instrum. 053, 301 (2005). https://doi.org/10.1063/1.1889465

Y. Futaana, S. Barabash, M. Wieser, P. Wurz, D. Hurley, M. Horányi, U. Mall, N. Andre, N. Ivchenko, J. Oberst, K. Retherford, A. Coates, A. Masters, J.E. Wahlund, E. Kallio, SELMA mission: how do airless bodies interact with space environment? The Moon as an accessible laboratory. Planet. Space Sci. 156, 23-40 (2018). https://doi.org/10.1016/j.pss.2017.11.002. Dust, Atmosphere, and Plasma Environment of the Moon and Small Bodies

A.B. Galvin, L.M. Kistler, M.A. Popecki, C.J. Farrugia, K.D.C. Simunac, L. Ellis, E. Möbius, M.A. Lee, M. Boehm, J. Carroll, A. Crawshaw, M. Conti, P. Demaine, S. Ellis, J.A. Gaidos, J. Googins, M. Granoff, A. Gustafson, D. Heirtzler, B. King, U. Knauss, J. Levasseur, S. Longworth, K. Singer, S. Turco, 
P. Vachon, M. Vosbury, M. Widholm, L.M. Blush, R. Karrer, P. Bochsler, H. Daoudi, A. Etter, J. Fischer, J. Jost, A. Opitz, M. Sigrist, P. Wurz, B. Klecker, M. Ertl, E. Seidenschwang, R.F. WimmerSchweingruber, M. Koeten, B. Thompson, D. Steinfeld, The Plasma and Suprathermal Ion Composition (PLASTIC) investigation on the STEREO observatories. Space Sci. Rev. 136(1), 437-486 (2008). https://doi.org/10.1007/s11214-007-9296-X

D.J. Gershman, J.A. Slavin, J.M. Raines, T.H. Zurbuchen, B.J. Anderson, H. Korth, D.N. Baker, S.C. Solomon, Ion kinetic properties in Mercury's pre-midnight plasma sheet. Geophys. Res. Lett. 41(16), 5740-5747 (2014). https://doi.org/10.1002/2014GL060468

D.J. Gershman, J.M. Raines, J.A. Slavin, T.H. Zurbuchen, T. Sundberg, S.A. Boardsen, B.J. Anderson, H. Korth, S.C. Solomon, MESSENGER observations of multiscale Kelvin-Helmholtz vortices at Mercury. J. Geophys. Res. Space Phys. 120(6), 4354-4368 (2015). https://doi.org/10.1002/2014JA020903

M. Grande, Investigation of magnetospheric interactions with the Hermean surface. Adv. Space Res. 19(10), 1609-1614 (1997). https://doi.org/10.1016/S0273-1177(97)00374-8. Proceedings of the BO.1 Symposium of COSPAR Scientific Commission B

H. Hasegawa, M. Fujimoto, T.D. Phan, H. Rème, A. Balogh, M.W. Dunlop, C. Hashimoto, R. TanDokoro, Transport of solar wind into Earth's magnetosphere through rolled-up Kelvin-Helmholtz vortices. Nature 430(7001), 755-758 (2004). https://doi.org/10.1038/nature02799

D. Heyner, C. Nabert, E. Liebert, K.H. Glassmeier, Concerning reconnection-induction balance at the magnetopause of Mercury. J. Geophys. Res. Space Phys. 121(4), 2935-2961 (2016). https://doi.org/10.1002/ 2015JA021484

G.C. Ho, D. Lario, R.B. Decker, C.W. Smith, Q. Hu, Transient shocks and associated energetic particle distributions observed by ACE during cycle 23. AIP Conf. Proc. 1039(1), 184-189 (2008). https://doi. org/10.1063/1.2982443

G.C. Ho, S.M. Krimigis, R.E. Gold, D.N. Baker, J.A. Slavin, B.J. Anderson, H. Korth, R.D. Starr, D.J. Lawrence, R.L. McNutt, S.C. Solomon, MESSENGER observations of transient bursts of energetic electrons in Mercury's magnetosphere. Science 333(6051), 1865-1868 (2011). https://doi.org/10. $1126 /$ science. 1211141

D. Hovestadt, M. Hilchenbach, A. Bürgi, B. Klecker, P. Laeverenz, M. Scholer, H. Grünwaldt, W.I. Axford, S. Livi, E. Marsch, B. Wilken, H.P. Winterhoff, F.M. Ipavich, P. Bedini, M.A. Coplan, A.B. Galvin, G. Gloeckler, P. Bochsler, H. Balsiger, J. Fischer, J. Geiss, R. Kallenbach, P. Wurz, K.U. Reiche, F. Gliem, D.L. Judge, H.S. Ogawa, K.C. Hsieh, E. Möbius, M.A. Lee, G.G. Managadze, M.I. Verigin, M. Neugebauer, CELIAS - charge, element and isotope analysis system for SOHO. Sol. Phys. 162(1), 441-481 (1995). https://doi.org/10.1007/BF00733436

A.J. Hundhausen, T.E. Holzer, B.C. Low, Do slow shocks precede some coronal mass ejections? J. Geophys. Res. Space Phys. 92(A10), 11,173-11,178 (1987). https://doi.org/10.1029/JA092iA10p11173

S.M. Imber, J.A. Slavin, MESSENGER observations of magnetotail loading and unloading: implications for substorms at Mercury. J. Geophys. Res. Space Phys. 122(11), 11,402-11,412 (2017). https://doi.org/10. 1002/2017JA024332

S.M. Imber, J.A. Slavin, S.A. Boardsen, B.J. Anderson, H. Korth, R.L. McNutt Jr., S.C. Solomon, MESSENGER observations of large dayside flux transfer events: do they drive Mercury's substorm cycle? J. Geophys. Res. Space Phys. 119(7), 5613-5623 (2014). https://doi.org/10.1002/2014JA019884

W.H. Ip, The sodium exosphere and magnetosphere of Mercury. Geophys. Res. Lett. 13(5), 423-426 (1986). https://doi.org/10.1029/GL013i005p00423

E. Kallio, P. Janhunen, Solar wind and magnetospheric ion impact on Mercury's surface. Geophys. Res. Lett. 30(17), 1877 (2003). https://doi.org/10.1029/2003GL017842

Y. Kasaba, T. Takashima, S. Matsuda, S. Eguchi, M. Endo, T. Miyabara, M. Taeda, Y. Kuroda, Y. Kasahara, T. Imachi, H. Kojima, S. Yagitani, M. Moncuquet, J.E. Wahlund, A. Kumamoto, A. Matsuoka, W. Baumjohann, S. Yokota, K. Asamura, Y. Saito, D. Delcourt, M. Hirahara, S. Barabash, N. Andre, M. Kobayashi, I. Yoshikawa, G. Murakami, H. Hayakawa, Mission data processor aboard the BepiColombo Mio spacecraft: design and scientific operation concept. Space Sci. Rev. 216(3), 34 (2020). https://doi.org/10.1007/s11214-020-00658-X

S. Kasahara, T. Mitani, K. Ogasawara, T. Takashima, M. Hirahara, K. Asamura, Application of single-sided silicon strip detector to energy and charge state measurements of medium energy ions in space. Nucl. Instrum. Methods Phys. Res., Sect. A, Accel. Spectrom. Detect. Assoc. Equip. 603(3), 355-360 (2009). https://doi.org/10.1016/j.nima.2009.02.004

Y. Kazama, S. Barabash, M. Wieser, K. Asamura, P. Wurz, A LENA instrument onboard BepiColombo and Chandrayaan-1. AIP Conf. Proc. 1144(1), 109-113 (2009). https://doi.org/10.1063/1.3169273

R.M. Killen, A.E. Potter, P. Reiff, M. Sarantos, B.V. Jackson, P. Hick, B. Giles, Evidence for space weather at Mercury. J. Geophys. Res., Planets 106(E9), 20,509-20,525 (2001). https://doi.org/10.1029/ 2000JE001401 
D. Lario, Advances in modeling gradual solar energetic particle events. Adv. Space Res. 36(12), 2279-2288 (2005). https://doi.org/10.1016/j.asr.2005.07.081. Space Weather

D. Lario, G.C. Ho, R.B. Decker, E.C. Roelof, M.I. Desai, C.W. Smith, ACE observations of energetic particles associated with transient interplanetary shocks. AIP Conf. Proc. 679(1), 640-643 (2003). https://doi.org/ 10.1063/1.1618676

B. Lavraud, J.E. Borovsky, Altered solar wind-magnetosphere interaction at low Mach numbers: coronal mass ejections. J. Geophys. Res. Space Phys. 113(A9), A00B08 (2008). https://doi.org/10.1029/ 2008JA013192

F. Leblanc, R. Johnson, Mercury exosphere I. Global circulation model of its sodium component. Icarus 209(2), 280-300 (2010). https://doi.org/10.1016/j.icarus.2010.04.020

E. Liljeblad, T. Sundberg, T. Karlsson, A. Kullen, Statistical investigation of Kelvin-Helmholtz waves at the magnetopause of Mercury. J. Geophys. Res. Space Phys. 119(12), 9670-9683 (2014). https://doi.org/ 10.1002/2014JA020614

S.T. Lindsay, M.K. James, E.J. Bunce, S.M. Imber, H. Korth, A. Martindale, T.K. Yeoman, MESSENGER $\mathrm{X}$-ray observations of magnetosphere-surface interaction on the nightside of Mercury. Planet. Space Sci. 125, 72-79 (2016). https://doi.org/10.1016/j.pss.2016.03.005

S. Machida, Y. Saito, Y. Ito, H. Hayakawa, Instrumental characteristics of the Electron Spectrum Analyzer (ESA) onboard the Planet-B mission and observational perspectives of the electron measurements. Earth Planets Space 50(3), 207-211 (1998). https://doi.org/10.1186/BF03352105

A. Marti, R. Schletti, P. Wurz, P. Bochsler, Calibration facility for solar wind plasma instrumentation. Rev. Sci. Instrum. 72(2), 1354-1360 (2001). https://doi.org/10.1063/1.1340020

S. Massetti, S. Orsini, A. Milillo, A. Mura, E.D. Angelis, H. Lammer, P. Wurz, Mapping of the cusp plasma precipitation on the surface of Mercury. Icarus 166(2), 229-237 (2003). https://doi.org/10.1016/j.icarus. 2003.08.005

A. Masters, T.D. Phan, S.V. Badman, H. Hasegawa, M. Fujimoto, C.T. Russell, A.J. Coates, M.K. Dougherty, The plasma depletion layer in Saturn's magnetosheath. J. Geophys. Res. Space Phys. 119(1), 121-130 (2014). https://doi.org/10.1002/2013JA019516

A. Milillo, P. Wurz, S. Orsini, D. Delcourt, E. Kallio, R.M. Killen, H. Lammer, S. Massetti, A. Mura, S. Barabash, G. Cremonese, I.A. Daglis, E. De Angelis, A.M. Di Lellis, S. Livi, V. Mangano, K. Torkar, Surface-exosphere-magnetosphere system of Mercury. Space Sci. Rev. 117(3), 397-443 (2005). https:// doi.org/10.1007/s11214-005-3593-Z

T. Mitani, T. Takashima, S. Kasahara, W. Miyake, M. Hirahara, High-energy electron experiments (HEP) aboard the ERG (ARASE) satellite. Earth Planets Space 70(1), 77 (2018). https://doi.org/10.1186/ s40623-018-0853-1

W. Miyake, Y. Saito, M. Harada, M. Saito, H. Hasegawa, A. Ieda, S. Machida, T. Nagai, T. Nagatsuma, K. Seki, I. Shinohara, T. Terasawa, Mercury Ion Analyzer (MIA) onboard Mercury Magnetospheric Orbiter: MMO. Adv. Space Res. 43(12), 1986-1992 (2009). https://doi.org/10.1016/j.asr.2009.03.011

T. Mukai, K. Ogasawara, Y. Saito, An empirical model of the plasma environment around Mercury. Adv. Space Res. 33(12), 2166-2171 (2004). https://doi.org/10.1016/S0273-1177(03)00443-5. Mercury, Mars and Saturn

D. Müller, R.G. Marsden, O.C. St Cyr, H.R. Gilbert (TSO Team), Solar orbiter. Sol. Phys. 285(1), 25-70 (2013). https://doi.org/10.1007/s11207-012-0085-7

A. Mura, P. Wurz, H.I. Lichtenegger, H. Schleicher, H. Lammer, D. Delcourt, A. Milillo, S. Orsini, S. Massetti, M.L. Khodachenko, The sodium exosphere of Mercury: comparison between observations during Mercury's transit and model results. Icarus 200(1), 1-11 (2009). https://doi.org/10.1016/j.icarus.2008. 11.014

T.K.M. Nakamura, H. Hasegawa, W. Daughton, S. Eriksson, W.Y. Li, R. Nakamura, Turbulent mass transfer caused by vortex induced reconnection in collisionless magnetospheric plasmas. Nat. Commun. 8(1), 1582 (2017). https://doi.org/10.1038/s41467-017-01579-0

N.F. Ness, K.W. Behannon, R.P. Lepping, Y.C. Whang, K.H. Schatten, Magnetic field observations near Mercury: preliminary results from Mariner 10. Science 185(4146), 151-160 (1974). https://doi.org/10. 1126/science.185.4146.151

M.N. Nishino, M. Fujimoto, T.D. Phan, T. Mukai, Y. Saito, M.M. Kuznetsova, L. Rastätter, Anomalous flow deflection at Earth's low-Alfvén-Mach-number bow shock. Phys. Rev. Lett. 101, 065,003 (2008). https:// doi.org/10.1103/PhysRevLett.101.065003

K.W. Ogilvie, J.D. Scudder, R.E. Hartle, G.L. Siscoe, H.S. Bridge, A.J. Lazarus, J.R. Asbridge, S.J. Bame, C.M. Yeates, Observations at Mercury encounter by the plasma science experiment on Mariner 10. Science 185(4146), 145-151 (1974). https://doi.org/10.1126/science.185.4146.145

M. Oka, F. Otsuka, S. Matsukiyo, L.B. Wilson, M.R. Argall, T. Amano, T.D. Phan, M. Hoshino, O.L. Contel, D.J. Gershman, J.L. Burch, R.B. Torbert, J.C. Dorelli, B.L. Giles, R.E. Ergun, C.T. Russell, P.A. Lindqvist, Electron scattering by low-frequency whistler waves at Earth's bow shock. Astrophys. J. 886(1), 53 (2019). https://doi.org/10.3847/1538-4357/ab4a81 
E. Parker, Dynamics of the interplanetary gas and magnetic fields. Astrophys. J. 128, 664 (1958). https://doi. org/10.1086/146579

Y. Saito, J. Sauvaud, M. Hirahara, S. Barabash, D. Delcourt, T. Takashima, K. Asamura, Scientific objectives and instrumentation of Mercury Plasma Particle Experiment (MPPE) onboard MMO. Planet. Space Sci. 58(1), 182-200 (2010a). https://doi.org/10.1016/j.pss.2008.06.003. Comprehensive Science Investigations of Mercury: The Scientific Goals of the Joint ESA/JAXA Mission BepiColombo

Y. Saito, S. Yokota, K. Asamura, T. Tanaka, M.N. Nishino, T. Yamamoto, Y. Terakawa, M. Fujimoto, H. Hasegawa, H. Hayakawa, M. Hirahara, M. Hoshino, S. Machida, T. Mukai, T. Nagai, T. Nagatsuma, T. Nakagawa, M. Nakamura, Ki. Oyama, E. Sagawa, S. Sasaki, K. Seki, I. Shinohara, T. Terasawa, H. Tsunakawa, H. Shibuya, M. Matsushima, H. Shimizu, F. Takahashi, In-flight performance and initial results of Plasma Energy Angle and Composition Experiment (PACE) on SELENE (Kaguya). Space Sci. Rev. 154(1), 265-303 (2010b). https://doi.org/10.1007/s11214-010-9647-x

Y. Saito, S. Yokota, K. Asamura, A. Krieger, High-speed MCP anodes for high time resolution low-energy charged particle spectrometers. J. Geophys. Res. Space Phys. 122(2), 1816-1830 (2017). https://doi.org/ 10.1002/2016JA023157

M. Sarantos, J.A. Slavin, On the possible formation of Alfvén wings at Mercury during encounters with coronal mass ejections. Geophys. Res. Lett. 36(4), L04107 (2009). https://doi.org/10.1029/2008GL036747

J.A. Sauvaud, A. Fedorov, C. Aoustin, H.C. Seran, E.L. Comte, M. Petiot, J. Rouzaud, Y. Saito, J. Dandouras, C. Jacquey, P. Louarn, C. Mazelle, J.L. Médale, The Mercury electron analyzers for the Bepi Colombo mission. Adv. Space Res. 46(9), 1139-1148 (2010). https://doi.org/10.1016/j.asr.2010.05.022

D. Schriver, P.M. Trávníček, B.J. Anderson, M. Ashour-Abdalla, D.N. Baker, M. Benna, S.A. Boardsen, R.E. Gold, P. Hellinger, G.C. Ho, H. Korth, S.M. Krimigis, R.L. McNutt Jr., J.M. Raines, R.L. Richard, J.A. Slavin, S.C. Solomon, R.D. Starr, T.H. Zurbuchen, Quasi-trapped ion and electron populations at Mercury. Geophys. Res. Lett. 38(23), L23103 (2011). https://doi.org/10.1029/2011GL049629

N. Shimada, T. Terasawa, M. Hoshino, T. Naito, H. Matsui, T. Koi, K. Maezawa, Diffusive shock acceleration of electrons at an interplanetary shock observed on 21 Feb 1994. Astrophys. Space Sci. 264(1), 481-488 (1998). https://doi.org/10.1023/A:1002499513777

I. Shinohara, M. Fujimoto, Formation of thin electron current layer associated with lower hybrid drift instability and its relation to quick reconnection triggering, in Frontiers in Magnetospheric Plasma Physics, ed. by M. Hoshohino, Y. Omura, L. Lanzerotti. COSPAR Colloquia Series, vol. 16 (Pergamon, Elmsford, 2005), pp. 123-129. https://doi.org/10.1016/S0964-2749(05)80019-8

P. Sigmund, A. Oliva, G. Falcone, Sputtering of multicomponent materials: elements of a theory. Nucl. Instrum. Methods Phys. Res. 194(1), 541-548 (1982). https://doi.org/10.1016/0029-554X(82)90578-X

J. Simpson, J. Eraker, J. Lamport, P. Walpole, Electrons and protons accelerated in Mercury's magnetic field. Science 185, 160-166 (1974). https://doi.org/10.1126/science.185.4146.160

J. Slavin, Mercury's magnetosphere. Adv. Space Res. 33(11), 1859-1874 (2004). https://doi.org/10.1016/j. asr.2003.02.019. Comparative Magnetospheres

J.A. Slavin, S.M. Imber, S.A. Boardsen, G.A. DiBraccio, T. Sundberg, M. Sarantos, T. Nieves-Chinchilla, A. Szabo, B.J. Anderson, H. Korth, T.H. Zurbuchen, J.M. Raines, C.L. Johnson, R.M. Winslow, R.M. Killen, R.L. McNutt Jr., S.C. Solomon, MESSENGER observations of a flux-transfer-event shower at Mercury. J. Geophys. Res. Space Phys. 117(A12), A00M06 (2012). https://doi.org/10.1029/ 2012JA017926

J.A. Slavin, D.N. Baker, D.J. Gershman, G.C. Ho, S.M. Imber, S.M. Krimigis, T. Sundberg, Mercury's dynamic magnetosphere, in Mercury: The View After MESSENGER. Cambridge Planetary Science (Cambridge University Press, Cambridge, 2018), pp. 461-496. https://doi.org/10.1017/9781316650684.018

J.A. Slavin, H.R. Middleton, J.M. Raines, X. Jia, J. Zhong, W.J. Sun, S. Livi, S.M. Imber, G.K. Poh, M. Akhavan-Tafti, J. Jasinski, G.A. DiBraccio, C. Dong, R.M. Dewey, M.L. Mays, MESSENGER observations of disappearing dayside magnetosphere events at Mercury. J. Geophys. Res. Space Phys. 124(8), 6613-6635 (2019). https://doi.org/10.1029/2019JA026892

D.F. Smart, M.A. Shea, A simplified model for timing the arrival of solar flare-initiated shocks. J. Geophys. Res. Space Phys. 90(A1), 183-190 (1985). https://doi.org/10.1029/JA090iA01p00183

W.J. Sun, J.M. Raines, S.Y. Fu, J.A. Slavin, Y. Wei, G.K. Poh, Z.Y. Pu, Z.H. Yao, Q.G. Zong, W.X. Wan, MESSENGER observations of the energization and heating of protons in the near-Mercury magnetotail. Geophys. Res. Lett. 44(16), 8149-8158 (2017). https://doi.org/10.1002/2017GL074276

B.T. Tsurutani, R.P. Lin, Acceleration of $>47 \mathrm{keV}$ ions and $>2 \mathrm{keV}$ electrons by interplanetary shocks at 1 AU. J. Geophys. Res. Space Phys. 90(A1), 1-11 (1985). https://doi.org/10.1029/JA090iA01p00001

P. van Nes, R. Reinhard, T.R. Sanderson, K.P. Wenzel, R.D. Zwickl, The energy spectrum of 35- to 1600-keV protons associated with interplanetary shocks. J. Geophys. Res. Space Phys. 89(A4), 2122-2132 (1984). https://doi.org/10.1029/JA089iA04p02122

C. Wang, D. Du, J.D. Richardson, Characteristics of the interplanetary coronal mass ejections in the heliosphere between 0.3 and 5.4 AU. J. Geophys. Res. Space Phys. 110(A10), A10107 (2005). https://doi. org/10.1029/2005JA011198 
S. Watanabe, H. Tajima, Y. Fukazawa, Y. Ichinohe, S. Takeda, T. Enoto, T. Fukuyama, S. Furui, K. Genba, K. Hagino, A. Harayama, Y. Kuroda, D. Matsuura, R. Nakamura, K. Nakazawa, H. Noda, H. Odaka, M. Ohta, M. Onishi, S. Saito, G. Sato, T. Sato, T. Takahashi, T. Tanaka, A. Togo, S. Tomizuka, The $\mathrm{Si} / \mathrm{CdTe}$ semiconductor Compton camera of the ASTRO-H Soft Gamma-Ray Detector (SGD). Nucl. Instrum. Methods Phys. Res., Sect. A, Accel. Spectrom. Detect. Assoc. Equip. 765, 192-201 (2014). https://doi.org/10.1016/j.nima.2014.05.127. hSTD-9 2013-Proceedings of the 9th International Hiroshima Symposium on Development and Application of Semiconductor Tracking Detectors

Y.C. Whang, Magnetospheric magnetic field of Mercury. J. Geophys. Res. 82(7), 1024-1030 (1977). https:// doi.org/10.1029/JA082i007p01024

M. Wieser, P. Wurz, Production of a $10 \mathrm{eV}-1000 \mathrm{eV}$ neutral particle beam using surface neutralization. Meas. Sci. Technol. 16(12), 2511-2516 (2005). https://doi.org/10.1088/0957-0233/16/12/016

R.M. Winslow, B.J. Anderson, C.L. Johnson, J.A. Slavin, H. Korth, M.E. Purucker, D.N. Baker, S.C. Solomon, Mercury's magnetopause and bow shock from MESSENGER magnetometer observations. J. Geophys. Res. Space Phys. 118(5), 2213-2227 (2013). https://doi.org/10.1002/jgra.50237

R.M. Winslow, N. Lugaz, L.C. Philpott, N.A. Schwadron, C.J. Farrugia, B.J. Anderson, C.W. Smith, Interplanetary coronal mass ejections from MESSENGER orbital observations at Mercury. J. Geophys. Res. Space Phys. 120(8), 6101-6118 (2015). https://doi.org/10.1002/2015JA021200

M. Wüest, D. Evans, ISS Institute, R. von Steiger, ES Agency, Calibration of Particle Instruments in Space Physics. ESA SR, ISSI (2007). The International Space Science Institute, https://books.google.co.jp/ books?id=hmBKNAAACAAJ

P. Wurz, H. Lammer, Monte-Carlo simulation of Mercury's exosphere. Icarus 164(1), 1-13 (2003). https:// doi.org/10.1016/S0019-1035(03)00123-4

M. Yagi, K. Seki, Y. Matsumoto, D.C. Delcourt, F. Leblanc, Formation of a sodium ring in Mercury's magnetosphere. J. Geophys. Res. Space Phys. 115(A10), A10253 (2010). https://doi.org/10.1029/ 2009JA015226

M. Yagi, K. Seki, Y. Matsumoto, D.C. Delcourt, F. Leblanc, Global structure and sodium ion dynamics in Mercury's magnetosphere with the offset dipole. J. Geophys. Res. Space Phys. 122(11), 10,990-11,002 (2017). https://doi.org/10.1002/2017JA024082

D.T. Young, J.J. Berthelier, M. Blanc, J.L. Burch, A.J. Coates, R. Goldstein, M. Grande, T.W. Hill, R.E. Johnson, V. Kelha, D.J. Mccomas, E.C. Sittler, K.R. Svenes, K. Szegö, P. Tanskanen, K. Ahola, D. Anderson, S. Bakshi, R.A. Baragiola, B.L. Barraclough, R.K. Black, S. Bolton, T. Booker, R. Bowman, P. Casey, F.J. Crary, D. Delapp, G. Dirks, N. Eaker, H. Funsten, J.D. Furman, J.T. Gosling, H. Hannula, C. Holmlund, H. Huomo, J.M. Illiano, P. Jensen, M.A. Johnson, D.R. Linder, T. Luntama, S. Maurice, K.P. Mccabe, K. Mursula, B.T. Narheim, J.E. Nordholt, A. Preece, J. Rudzki, A. Ruitberg, K. Smith, S. Szalai, M.F. Thomsen, K. Viherkanto, J. Vilppola, T. Vollmer, T.E. Wahl, M. Wüest, T. Ylikorpi, C. Zinsmeyer, Cassini plasma spectrometer investigation. Space Sci. Rev. 114(1), 1-112 (2004). https://doi.org/10. 1007/s11214-004-1406-4

T.H. Zurbuchen, J.M. Raines, J.A. Slavin, D.J. Gershman, J.A. Gilbert, G. Gloeckler, B.J. Anderson, D.N. Baker, H. Korth, S.M. Krimigis, M. Sarantos, D. Schriver, R.L. McNutt, S.C. Solomon, MESSENGER observations of the spatial distribution of planetary ions near Mercury. Science 333(6051), 1862-1865 (2011). https://doi.org/10.1126/science.1211302

Publisher's Note Springer Nature remains neutral with regard to jurisdictional claims in published maps and institutional affiliations.

\section{Authors and Affiliations}

Yoshifumi Saito ${ }^{1}$ (D) - Dominique Delcourt ${ }^{2}$ - Masafumi Hirahara ${ }^{3} \cdot$ Stas Barabash $^{4}$.

Nicolas André ${ }^{5}$ Takeshi Takashima' ${ }^{1}$ Kazushi Asamura' ${ }^{1}$ Shoichiro Yokota ${ }^{6}$.

Martin Wieser ${ }^{4}$. Masaki N. Nishino ${ }^{1}$. Mitsuo Oka ${ }^{7}$. Yoshifumi Futaana ${ }^{4}$.

Yuki Harada ${ }^{8}$. Jean-André Sauvaud ${ }^{5}$. Philippe Louarn ${ }^{5}$. Benoit Lavraud ${ }^{5}$.

Vincent Génot ${ }^{5}$. Christian Mazelle ${ }^{5}$. lannis Dandouras ${ }^{5}$. Christian Jacquey ${ }^{5}$. Claude Aoustin ${ }^{5}$. Alain Barthe ${ }^{9} \cdot$ Alexandre Cadu $^{10}$. Andréi Fedorov ${ }^{5}$.

Anne-Marie Frezoul ${ }^{11}$. Catherine Garat ${ }^{12}$. Eric Le Comte ${ }^{5}$. Qiu-Mei Lee ${ }^{5}$.

Jean-Louis Médale ${ }^{5}$. David Moirin ${ }^{12}$. Emmanuel Penou ${ }^{5}$. Mathieu Petiot ${ }^{5}$. Guy Peyre $^{13} \cdot$ Jean Rouzaud ${ }^{5} \cdot$ Henry-Claude Séran $^{5} \cdot$ Zdeněk Němeček $^{14}$. 
Jana Šafránková ${ }^{14}$. Maria Federica Marcucci ${ }^{15} \cdot$ Roberto Bruno $^{15}$. Giuseppe Consolini ${ }^{15}$. Wataru Miyake ${ }^{16}$. Iku Shinohara ${ }^{1} \cdot$ Hiroshi Hasegawa ${ }^{1}$. Kanako Seki ${ }^{17}$. Andrew J. Coates ${ }^{18} \cdot$ Frédéric Leblanc $^{2} \cdot$ Christophe Verdeil $^{5}$. Bruno Katra $^{2}$. Dominique Fontaine ${ }^{19}$. Jean-Marie Illiano ${ }^{2}$. Jean-Jacques Berthelier ${ }^{20}$. Jean-Denis Techer ${ }^{2}$. Markus Fraenz ${ }^{21}$. Henning Fischer ${ }^{21}$. Norbert Krupp ${ }^{21}$. Joachim Woch ${ }^{21}$. Ulrich Bührke ${ }^{21}$. Björn Fiethe ${ }^{22}$. Harald Michalik ${ }^{22}$. Haruhisa Matsumoto ${ }^{23}$. Tomoki Yanagimachi ${ }^{24}$. Yoshizumi Miyoshi ${ }^{3}$. Takefumi Mitani ${ }^{1}$ - Manabu Shimoyama ${ }^{4}$ Qiugang Zong ${ }^{25}$. Peter Wurz ${ }^{26}$. Herman Andersson ${ }^{4}$. Stefan Karlsson ${ }^{4}$. Mats Holmström ${ }^{4}$. Yoichi Kazama ${ }^{27}$. Wing-Huen $\mathrm{Ip}^{28}$. Masahiro Hoshino ${ }^{17}$. Masaki Fujimoto ${ }^{1}$. Naoki Terada ${ }^{29}$. Kunihiro Keika ${ }^{17}$. BepiColombo Mio/MPPE Team ${ }^{30}$

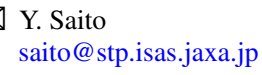
LATMOS-CNRS-IPSL, 4 place Jussieu, 75252 Paris, France MPS, Justus-von-Liebig Weg 3, 37077 Goettingen, Germany IDA, Hans-Sommer-Str. 66, 38106 Braunschweig, Germany JAXA, Sengen 2chome, Tsukuba, Ibaraki 305-8505, Japan Rikkyo University, 3-34-1 Nishi-Ikebukuro, Toshima-ku, Tokyo 171-8501, Japan Peking University, North Physics Building, Peking University, Beijing, 100871, China 26 University of Bern, 3012, Bern, Switzerland 
“SPAC 11214 layout: Small Condensed file: spac839.tex (karolis.kavaliauskas) class: spr-small-v1 2020/06/02 v2.07 Prn:22/07/2021; 13:21 p. 91/91» doctopic: ReviewPaper numbering style: ContentOnly reference style: aps"

4501

4502

4503

4504

4505

4506

4507

4508

4509

4510

4511

4512

4513

4514

4515

4516

4517

4518

4519

4520

4521

4522

4523

4524

4525

4526

4527

4528

4529

4530

4531

4532

4533

4534

4535

4536

4537

4538

4539

4540

4541

4542

4543

4544

4545

4546

4547

4548

4549

4550
27 Academia Sinica Institute of Astronomy and Astrophysics, No. 1, Sect. 4, Roosevelt Rd, Taipei 10617, Taiwan

28 IASS, National Central University, Chung-Li, 32054 Taiwan

29 Tohoku University, Aramaki-aza-aoba, Aoba-ku, Sendai, Miyagi 980-8578, Japan

30 https://www.cosmos.esa.int/web/bepicolombo/mppe 